\title{
HOLONOMY GROUPS IN A TOPOLOGICAL CONNECTION THEORY
}

\author{
By
}

\author{
Kensaku KITADA
}

\begin{abstract}
We study slicing functions, which are called direct connections in the smooth category, and parallel displacements along sequences in a topological connection theory. We define holonomy groups for such parallel displacements, and prove a holonomy reduction theorem and related results. In particular, we study a category of principal bundles with parallel displacements over a fixed base space. Assuming the existence of an initial object of a category of principal $G$-bundles, we obtain a classification theorem of topological principal $G$-bundles in terms of topological group homomorphisms. It is shown that a certain object is an initial object if it is the holonomy reduction of itself with respect to the identification topology. The result is applied to the universal bundle over a countable simplicial complex constructed by Milnor.
\end{abstract}

\section{Introduction}

Connection theory has been one of the most important branch in differential geometry. Recently, various results have been obtained also in gauge theory. On the other hand, as known in a series of works relevant to collapsing Riemannian manifolds, the limit space of the Gromov-Hausdorff convergence of Riemannian manifolds is not a manifold in general (see $[6,8,15])$. Thus, it seems to be convenient to study a topological connection theory which is still effective also in more general spaces including such limit ones. In a category of certain topological spaces, generalized or analogous theories of connection theory have been studied.

2000 Mathematics Subject Classification: Primary 53C05; Secondary 53C29, 55R15, 54A10.

Key words and phrases: Slicing function; direct connection; parallel displacement; holonomy group; classification theorem.

Received July 19, 2012.

Revised April 30, 2013. 
For example, N. Abe [1] generalized parallel displacements along piecewise smooth curves to parallel displacements along admissible paths, defined a holonomy group, and proved a holonomy reduction theorem. A. Asada $[3,4,5]$ generalized connection theory to topological fiber bundles using the AlexanderSpanier cohomology and obtained various results. A connection in the sense of Asada is the germ of a slicing function at the diagonal set of the base space of a $G$-bundle. Slicing functions have been studied in the theory of fibrations ([2, 9]). Recently, J. Kubarski and N. Teleman $[13,16]$ have studied a smooth slicing function, which they call a linear direct connection in smooth vector bundles, whose infinitesimal part is shown to be a linear connection ([13, Theorem 3]). They showed that the Chern character of smooth vector bundles can be represented as the periodic cyclic homology class of a specific cyclic cycle, manufactured from a direct connection. Direct connections have been used without systematic study for several constructions in $K$-theory and cyclic homology ([7, 11]). J. Milnor [14] considered slicing functions defined on any subset of $X \times X$ containing the diagonal set $\Delta_{X}$, not necessarily a neighborhood of $\Delta_{X}$. He constructed a universal bundle over a polyhedron $X$ of a countable connected simplicial complex $K$ in the weak topology. Moreover, he showed that any principal bundle over $X$ associates with this universal bundle ([14, Theorem 5.1]) by constructing slicing functions.

The purpose of this paper is to define, in a topological connection theory, slicing functions, parallel displacements along sequences, and their holonomy groups as an analogue of those in [1], and study their fundamental properties. The main results are holonomy reduction theorems (Theorems 6.3 and 7.5), and a classification theorem (Theorem 8.1).

In Section 2, we recall some properties of topological bundles, especially principal $G$-bundles, following mostly Husemoller [10].

In Section 3, we introduce slicing functions in topological bundles, which induce parallel displacements in our sense in the next section. We also give examples, some of which indicate that the slicing function is a generalization of the connection in the smooth category. In particular, it is shown that connections in the smooth category induce smooth slicing functions. Moreover, we generalize Theorem 3 in [13] to a smooth principal $G$-bundle.

In Section 4, we define parallel displacements along admissible sequences as an analogue of those along admissible paths in [1]. Moreover, we define morphisms which preserve the parallel displacements and study some properties of them.

In Section 5, we define a holonomy group of the parallel displacement as an analogue of that of the parallel displacement along admissible paths in [1]. We 
study fundamental properties of the holonomy groups. In particular, a relation between the holonomy group of a connection in the smooth category and that of the parallel displacement induced by the smooth slicing function in Section 3 is studied. Moreover, we define a local holonomy group of the parallel displacement. In the smooth category, the Lie algebra of the holonomy group of a connection is spanned by the image of the curvature. As an analogue of this fact, we show that the local holonomy group is generated by a curvature of the parallel displacement, whose germ is the curvature in the sense of Asada [4, 5]. At the end of this section, we study a relation between the local holonomy group of a connection in the smooth category and the local holonomy group of the parallel displacement induced by the smooth slicing function in Section 3.

In Section 6, we define a holonomy bundle of the parallel displacement as an analogue of that of the parallel displacement along admissible paths in [1] and study their fundamental properties. In particular, we obtain a holonomy reduction theorem (Theorem 6.3) and related results as in the smooth category and [1].

In Section 7, we define a strong holonomy group whose topology is the identification topology induced by a certain monoid homomorphism. Especially, we introduce a concept of strong holonomy reduction and obtain a strong version of the holonomy reduction theorem (Theorem 7.5).

Finally, in Section 8, we study a category of principal bundles with parallel displacements over a fixed base space. We obtain a classification theorem (Theorem 8.1) in the following sense. If there exists an initial object of a category of principal $G$-bundles and parallel displacements, the bundles with parallel displacements are classified in terms of topological group homomorphisms from the structure group of the initial object to those of bundles. We obtain a sufficient condition for the existence of an initial object. We see that a certain object is an initial object if it is the strong holonomy reduction of itself. From this fact, it follows that the universal bundle constructed by Milnor over $X$ together with the parallel displacement induced by a certain slicing function is an initial object in the category. In particular, Theorem 5.1 in [14] follows.

\section{Preliminaries}

At first we prepare notations of maps and some topological facts. Let $f: X \rightarrow Y$ be a map and $A \subset X, B \subset Y$ such that $f(A) \subset B$. Then there exists a unique map $k: A \rightarrow B$ such that $f \circ i_{A, X}=i_{B, Y} \circ k$, where $i_{A, X}$ is the inclusion. We denote by ${ }_{B}|f|_{A}$ the map $k$. Moreover, we denote by $\left.f\right|_{A}$ (resp. ${ }_{B} \mid f$ ) the map 
${ }_{Y}|f|_{A}$ (resp. ${ }_{B}|f|_{X}$ ). If there is no confusion, we denote by $\left.f\right|_{A}$ or $f: A \rightarrow B$ the map ${ }_{B}|f|_{A}$ as usual. Let $f: X \rightarrow Y, g: Z \rightarrow W$ be maps. We denote the composition $\left(\left.g\right|_{Y \cap Z}\right) \circ\left(Y \cap Z|f|_{f^{-1}(Y \cap Z)}\right)$ simply by $g \circ f$. Note that if $Y \cap Z=\varnothing$, then $g \circ f: \varnothing \rightarrow W$. Suppose that $X=Z$ and let $\delta: X \rightarrow X \times X$ be a diagonal map. We denote by $f \hat{\times} g$ the composition $(f \times g) \circ \delta: X \rightarrow Y \times W$. Explicitly, $(f \hat{\times} g)(x)=(f(x), g(x))$ for $x \in X$. If $X$ and $Y$ are topological spaces and $f: X \rightarrow Y$ is a continuous map, then ${ }_{B}|f|_{A}$ is also continuous with respect to the relative topologies. We call $f$ an identification if the topology of $Y$ is $\left\{U \in \mathscr{P}(Y) \mid f^{-1}(U) \in \mathcal{O}_{X}\right\}$, that is, the identification topology with respect to $f$, where $\mathscr{P}(Y)$ is the power set of $Y$ and $\mathcal{O}_{X}$ is the topology of $X$. Note that a surjective continuous open map is an identification. The following lemmas are frequently used in this paper.

Lemma 2.1. A surjective map $f: X \rightarrow Y$ is an identification if and only if $f(A)|f|_{A}: A \rightarrow f(A)$ is also an identification for any open (or closed) subset $A \subset X$ such that $f^{-1}(f(A))=A$.

Lemma 2.2. A map $f: X \rightarrow Y$ is an open map if and only if ${ }_{A}|f|_{f^{-1}(A)}$ : $f^{-1}(A) \rightarrow A$ is an open map for any subset $A \subset Y$.

We mostly follow the terminology of [10] with slight changes in notation. Thus, hereafter in this section, we set up notation for bundles. For a continuous map $\pi: E \rightarrow X$, we call the map $\pi: E \rightarrow X$ itself a bundle while usually the triple $\xi=(E, \pi, X)$ or the total space $E$ is referred to as a bundle. Let $\pi: E \rightarrow X$ and $\pi^{\prime}: E^{\prime} \rightarrow X^{\prime}$ be bundles. For continuous maps $h: E \rightarrow E^{\prime}$ and $f: X \rightarrow X^{\prime}$, we call $(h, f): \pi \rightarrow \pi^{\prime}$ a bundle morphism if $\pi^{\prime} \circ h=f \circ \pi$. We denote by $\operatorname{Hom}\left(\pi, \pi^{\prime}\right)$ (resp. Iso $\left.\left(\pi, \pi^{\prime}\right)\right)$ the set of bundle morphisms (resp. isomorphisms). If $X=X^{\prime}$, we call $\left(h, i d_{X}\right): \pi \rightarrow \pi^{\prime}$ an $X$-morphism and denote it simply by $h$. We denote by $\operatorname{Hom}_{X}\left(\pi, \pi^{\prime}\right)$ (resp. $\left.\operatorname{Iso}_{X}\left(\pi, \pi^{\prime}\right)\right)$ the set of $X$-morphisms (resp. $X$-isomorphisms). For $Y \subset X$, put $E\left\lceil_{Y}:=\pi^{-1}(Y)\right.$ and $\pi\left\lceil_{Y}:={ }_{Y}|\pi|_{\pi^{-1}(Y)}\right.$, then we call $\pi\left\lceil_{Y}: E\left\lceil_{Y}\right.\right.$ $\rightarrow Y$ the restricted bundle of $\pi$ to $Y$. For a continuous map $f: Z \rightarrow X$, the induced bundle or pull-back of $\pi$ is denoted by $f^{*} \pi: f^{*} E \rightarrow Z$, where

$$
f^{*} E:=Z \times{ }_{X} E:=\{(z, u) \in Z \times E \mid f(z)=\pi(u)\}
$$

is a fiber product of $Z \stackrel{f}{\rightarrow} X \stackrel{\pi}{\leftarrow} E$. The canonical bundle map is denoted by $(\bar{f}, f)$. For topological spaces $X$ and $F$, a bundle $\operatorname{pr}_{1}: X \times F \rightarrow X$ is called a product bundle. If $\pi$ is $X$-isomorphic to a product bundle, we say that $\pi$ is trivial. We say 
that $\pi: E \rightarrow X$ is locally trivial if $\pi$ is locally $V$-isomorphic to a product bundle $\mathrm{pr}_{1}: V \times F \rightarrow V$ for some open subset $V \subset X$. An element of $\operatorname{Iso}_{V}\left(\pi\left\lceil_{V}, \operatorname{pr}_{1}\right)\right.$ is called a local trivialization.

We recall $G$-spaces and $G$-bundles. Let $G$ be a topological group. A right $G$-space is a topological space $E$ equipped with a continuous right action $\mu: E \times G \rightarrow E$. We often denote $\mu(u, a)$ simply by $u a$. For $u \in E$ and $a \in G$, two maps $l_{u}: G \rightarrow E$ and $r_{a}: E \rightarrow E$ are given by $l_{u}(b):=u b$ and $r_{a}(v):=v a$ respectively. A left $G$-space is defined in the same way. A $G$-space is a right $G$-space unless otherwise mentioned. We call $E$ a free $G$-space if the right action is free. We denote by $E / G$ the orbit space, and by $q_{G}^{E}: E \rightarrow E / G$ the natural projection, where the topology of $E / G$ is the identification topology (that is, the quotient topology) induced by $q_{G}^{E}$. Note that $q_{G}^{E}$ is a surjective open map. A translation function $T: E^{*} \rightarrow G$ is a (not necessarily continuous) map such that $u T(u, v)=v$ for any $(u, v) \in E^{*}$, where

$$
E^{*}:=\left\{(u, u a) \in E^{2} \mid a \in G\right\} .
$$

Let $E$ be a free $G$-space. Since for any $(u, v) \in E^{*}$ there exists a unique $a \in G$ such that $v=u a$, a translation function $T: E^{*} \rightarrow G$ is given by $T(u, v):=a$. We have the following:

(1) For any $u \in E, T(u, u)=1_{G}$.

(2) For any $(u, v) \in E^{*}$ and $(a, b) \in G^{2}, \quad(u a, v b) \in E^{*}$ and $T(u a, v b)=$ $a^{-1} T(u, v) b$.

(3) For any $(u, v, w) \in E^{3}$ such that $(u, v),(v, w) \in E^{*}, \quad T(u, v) T(v, w)=$ $T(u, w)$.

We call $E$ a principal $G$-space if $T$ is continuous. Let $\pi: E \rightarrow X$ be a bundle such that $E$ is a $G$-space. We call $\pi$ a $G$-bundle if $q_{G}^{E}$ and $\pi$ are isomorphic by $\left(i d_{E}, f\right)$, where $f$ is a unique continuous map such that $f \circ q_{G}^{E}=\pi \circ i d_{E}$. We denote by $\pi /{ }^{G}$ the map $f$. The following lemma provides a rather practical condition for a bundle to be a $G$-bundle.

Lemma 2.3. Let $\pi$ be a bundle whose total space is a G-space. Then $\pi$ is a G-bundle if and only if the map $\pi /^{G}$ is well-defined and a homeomorphism.

Let $\pi$ (resp. $\pi^{\prime}$ ) be a $G$ (resp. $\left.G^{\prime}\right)$-bundle. For a continuous group homomorphism $\rho: G \rightarrow G^{\prime}$ and a bundle morphism $(h, f): \pi \rightarrow \pi^{\prime}$, we call a triple $(h, f, \rho):(\pi, G) \rightarrow\left(\pi^{\prime}, G^{\prime}\right)$ a homomorphism if $h(u a)=h(u) \rho(a)$ for $(u, a) \in E \times G$. If $G=G^{\prime}$, we call $\left(h, f, i d_{G}\right)$ a $G$-morphism and denote it simply by $(h, f)$. 
We denote by $\operatorname{Hom}\left((\pi, G),\left(\pi^{\prime}, G^{\prime}\right)\right)$ (resp. $\left.\operatorname{Hom}_{G}\left((\pi, G),\left(\pi^{\prime}, G\right)\right)\right)$ the set of homomorphisms (resp. $G$-morphisms). If $\operatorname{cod} \pi=\operatorname{cod} \pi^{\prime}=X$, put

$$
\begin{aligned}
\operatorname{Hom}_{X}\left((\pi, G),\left(\pi^{\prime}, G^{\prime}\right)\right) & :=\left\{(h, \rho) \in \operatorname{Hom}\left((\pi, G),\left(\pi^{\prime}, G^{\prime}\right)\right) \mid h \in \operatorname{Hom}_{X}\left(\pi, \pi^{\prime}\right)\right\}, \\
\operatorname{Hom}_{X, G}\left((\pi, G),\left(\pi^{\prime}, G\right)\right) & :=\left\{h \in \operatorname{Hom}_{X}\left(\pi, \pi^{\prime}\right) \mid\left(h, i d_{X}\right) \in \operatorname{Hom}_{G}\left((\pi, G),\left(\pi^{\prime}, G\right)\right)\right\} .
\end{aligned}
$$

The set of isomorphisms is obtained by replacing Hom by Iso. We call an element of $\operatorname{Hom}_{X, G}\left((\pi, G),\left(\pi^{\prime}, G\right)\right)$ an $(X, G)$-morphism. We call a $G$-bundle $\pi: E \rightarrow X$ a principal $G$-bundle if $E$ is a principal $G$-space.

Let $\pi: E \rightarrow X$ be a principal $G$-bundle. Using Lemma 2.2, we can see that the restricted bundle $\pi \Gamma_{Y}$ is a principal $G$-bundle. The induced bundle $f^{*} \pi$ is a principal $G$-bundle in the natural way.

Here we recall associated bundles. Let $G$ and $G^{\prime}$ be topological groups, $\rho: G \rightarrow G^{\prime}$ a continuous group homomorphism, and $E$ a $G$-space. The product space $E \times G^{\prime}$ is a $G$-space by a right action $(u, a) b:=\left(u b, \rho(b)^{-1} a\right)$. We denote by $E^{\rho}$ the orbit space $\left(E \times G^{\prime}\right) / G$. The orbit space $E^{\rho}$ is a $G^{\prime}$-space by a right action $[u, b] c:=[u, b c]$. We can see that this action is continuous. We call $E^{\rho}$ a $G^{\prime}$-space associated with $E$. If $E$ is a free $G$-space, then $E^{\rho}$ is a free $G^{\prime}$-space. If $E$ is a principal $G$-space with the translation function $T$, then $E^{\rho}$ is a principal $G^{\prime}$-space with the translation function given by

$$
T^{\rho}([u, a],[v, b]):=a^{-1} \rho(T(u, v)) b
$$

for $([u, a],[v, b]) \in\left(E^{\rho}\right)^{*}$. Using Lemma 2.2, we can see that $T^{\rho}$ is continuous. Let $\pi: E \rightarrow X$ be a principal $G$-bundle. Let $\pi^{\rho}: E^{\rho} \rightarrow X$ be the map such that $\pi^{\rho} \circ q_{G}^{E \times G^{\prime}}=\pi \circ \mathrm{pr}_{1}$. We can see that $\pi^{\rho}$ is a principal $G^{\prime}$-bundle. We call $\pi^{\rho}$ the principal $G^{\prime}$-bundle associated with $\pi$. A map $\theta^{\rho}: E \rightarrow E^{\rho}$ is given by

$$
\theta^{\rho}(u):=\left[u, 1_{G^{\prime}}\right]
$$

for $u \in E$. Then we can see $\left(\theta^{\rho}, \rho\right) \in \operatorname{Hom}_{G}\left((\pi, G),\left(\pi^{\rho}, G^{\prime}\right)\right)$. Let $\pi^{\prime}: E^{\prime} \rightarrow X$ be a principal $G^{\prime}$-bundle and $(h, \rho) \in \operatorname{Hom}_{X}\left((\pi, G),\left(\pi^{\prime}, G^{\prime}\right)\right)$. A map $h^{\rho}: E^{\rho} \rightarrow E^{\prime}$ is given by

$$
h^{\rho}([u, a]):=h(u) a
$$

for $[u, a] \in E^{\rho}$. Then we can see that $h^{\rho} \in \operatorname{Hom}_{X, G^{\prime}}\left(\left(\pi^{\rho}, G^{\prime}\right),\left(\pi^{\prime}, G^{\prime}\right)\right)$ and $h=$ $h^{\rho} \circ \theta^{\rho}$. From Theorem 3.2 and the succeeding observation in [10], Chapter 4, we have the following lemma. 
Lemma 2.4. If $\pi^{\prime}$ is a principal $G^{\prime}$-bundle, then $h^{\rho} \in \operatorname{Iso}_{X, G^{\prime}}\left(\left(\pi^{\rho}, G^{\prime}\right),\left(\pi^{\prime}, G^{\prime}\right)\right)$.

Next, we recall local triviality of $G$-bundles. Let $E \stackrel{\pi}{\rightarrow} X$ be a $G$-bundle. We say that $\pi$ is locally $G$-trivial or simply locally trivial if $\pi$ is locally $(V, G)$ isomorphic to a product $G$-bundle $\mathrm{pr}_{1}: V \times G \rightarrow V$ for some open subset $V \subset X$. An element of $\operatorname{Iso}_{V, G}\left(\left(\pi \Gamma_{V}, G\right),\left(\operatorname{pr}_{1}, G\right)\right)$ is called a local trivialization. For a local trivialization $\alpha:\left(\pi\left\lceil_{V}, G\right) \rightarrow\left(\operatorname{pr}_{1}, G\right)\right.$, put $U_{\alpha}:=V$. For local trivializations $\alpha$ and $\beta$, the transition function $g_{\alpha \beta}: U_{\alpha} \cap U_{\beta} \rightarrow G$ is given by

$$
g_{\alpha \beta}(x):=\left(\mathrm{pr}_{2} \circ \alpha \circ \beta^{-1}\right)\left(x, 1_{G}\right) .
$$

Note that a locally trivial $G$-bundle is a principal $G$-bundle. For a local trivialization $\alpha$, let $s_{\alpha}: U_{\alpha} \rightarrow E\left\lceil_{U_{\alpha}}\right.$ be the local section given by $s_{\alpha}(x):=\alpha^{-1}\left(x, 1_{G}\right)$. Then $T \circ\left(s_{\alpha} \hat{x} s_{\beta}\right)=g_{\alpha \beta}$ holds. If $\pi$ is a locally trivial $G$-bundle, we can see that $\pi\left\lceil_{Y}, f^{*} \pi, \pi^{\rho}\right.$ are locally trivial respectively.

\section{Slicing Functions and Several Examples}

In this section, we introduce slicing functions in, not necessarily locally $G$-trivial, bundles and give several examples. Some of them indicate that the slicing function is a generalization of the connection in the smooth category. In Section 4, we shall see that any slicing function induces a parallel displacement in a natural manner.

Let $E \stackrel{\pi}{\rightarrow} X$ be a bundle, $\Delta_{X}$ the diagonal set of $X$, and $U \subset X^{2}$ such that $\Delta_{X} \subset U$. For $i \in\{0,1\}$, let $p_{i}^{(1)}: X^{2} \rightarrow X$ be a projection defined by $p_{i}^{(1)}\left(x_{1}, x_{0}\right)$ $:=x_{i}$ for $\left(x_{1}, x_{0}\right) \in X^{2}$.

Definition 3.1 (cf. [14]). Let $\omega:\left(\left.p_{0}^{(1)}\right|_{U}\right)^{*} E \rightarrow E$ be a continuous map. Put $\omega_{x, y}:=\omega(x, y, \cdot): E_{y} \rightarrow E$ for $(x, y) \in U$. We call $\omega$ a slicing function in $\pi$ over $U$ if it satisfies the following conditions:

(1) $\left(\omega,\left.p_{1}^{(1)}\right|_{U}\right) \in \operatorname{Hom}\left(\left(\left.p_{0}^{(1)}\right|_{U}\right)^{*} \pi, \pi\right)$.

(2) $\omega_{x, x}=i d_{E_{x}}$ for any $x \in X$.

Let $U$ be symmetric, that is, $(y, x) \in U$ for any $(x, y) \in U$. A slicing function $\omega$ is said to be invertible if it satisfies the following condition:

$$
\omega_{y, x}=\omega_{x, y}^{-1} \text { for any }(x, y) \in U .
$$

Let $\pi$ be a $G$-bundle. We say that $\omega$ is $G$-compatible if

$$
\left(\omega,\left.p_{1}^{(1)}\right|_{U}\right) \in \operatorname{Hom}_{G}\left(\left(\left(\left.p_{0}^{(1)}\right|_{U}\right)^{*} \pi, G\right),(\pi, G)\right) .
$$


We denote by $S F(\pi, U)$ (resp. $S F_{\text {inv }}(\pi, U), S F(\pi, U)_{G}$ ) the set of slicing functions (resp. invertible slicing functions, $G$-compatible slicing functions) on $\pi$ over $U$. Put

$$
S F_{\text {inv }}(\pi, U)_{G}:=S F_{\text {inv }}(\pi, U) \cap S F(\pi, U)_{G} .
$$

Note that the definition of invertible $G$-compatible slicing function coincides with that of Milnor's if the $G$-bundle is locally $G$-trivial.

REMARK 3.1. Let $i d_{X}^{*} \pi: i d_{X}^{*} E \rightarrow X$ be the induced bundle of $\pi$ by $i d_{X}$. Let $\left(\overline{i d}_{X}, i d_{X}\right): i d_{X}^{*} \pi \rightarrow \pi$ be the canonical bundle map and $\left({ }_{U}\left|\delta \times i d_{E},{ }_{U}\right| \delta\right): i d_{X}^{*} \pi$ $\rightarrow\left(\left.p_{0}^{(1)}\right|_{U}\right)^{*} \pi$ the bundle map, where $\delta: X \rightarrow X^{2}$ is the diagonal map. For a continuous map $\omega:\left(\left.p_{0}^{(1)}\right|_{U}\right)^{*} E \rightarrow E, \omega \in S F(\pi, U)$ if and only if the following diagram commutes:



A preordered set $I$ is said to be pseudodirected set if any finite subset of it is bounded above. Let $I$ be a pseudodirected set and $\left(U_{i}\right)_{i \in I}$ a system of subsets of $X^{2}$ such that for any $i, j \in I, \Delta_{X} \subset U_{i}$ and if $i \leq j$, then $U_{j} \subset U_{i}$. For $i, j \in I$ with $i \leq j$, let $\rho_{j i}: S F\left(\pi, U_{i}\right) \rightarrow S F\left(\pi, U_{j}\right)$ be the restriction, that is, $\rho_{j i}(\omega)=\left.\omega\right|_{\left(\left.p_{0}^{(1)}\right|_{U_{j}}\right)^{*} E}$ for $\omega \in S F\left(\pi, U_{i}\right)$. Then $\left(S F\left(\pi, U_{i}\right), \rho_{j i}\right)_{(i, j) \in I^{2}}$ is an inductive system. We denote by $[\omega]_{I}$ an element of $\lim _{\rightarrow} S F\left(\pi, U_{i}\right)$. Similarly we have the inductive limits of $S F_{\text {inv }}\left(\pi, U_{i}\right), S F\left(\pi, U_{i}\right)_{G}$, and $S F_{\text {inv }}\left(\pi, U_{i}\right)_{G}$. For example, let $\mathcal{O}_{X^{2}}\left(\Delta_{X}\right)$ denote the set of open neighborhoods of $\Delta_{X}$ in $X^{2}$, partially ordered by $U \leq V$ if $V \subset U$, and let a system of subset of $X^{2}$ be $i_{\mathcal{O}_{X^{2}}\left(\Delta_{X}\right), \mathscr{P}\left(X^{2}\right)}=(U)_{U \in \mathcal{O}_{X^{2}}\left(\Delta_{X}\right)}$. Then we have $[\omega]_{\mathcal{O}_{X^{2}}\left(\Delta_{X}\right)} \in \lim _{\longrightarrow} S F(\pi, U)$.

We have two types of trivial examples of slicing functions.

Example 3.1. Let $\mathrm{pr}_{1}: X \times F \rightarrow X$ be the product bundle. Put

$$
\omega(x, y,(y, p)):=(x, p)
$$

for $(x, y,(y, p)) \in p_{0}^{(1) *}(X \times F)$. Then, $\omega \in S F_{\text {inv }}\left(\operatorname{pr}_{1}, X \times X\right)$. Let $G$ be a topological group and $F=G$. Then $\omega \in S F_{\text {inv }}\left(\operatorname{pr}_{1}, X \times X\right)_{G}$. 
Example 3.2. Let $\pi: E \rightarrow X$ be a bundle. Put

$$
\omega(x, x, u):=u
$$

for $(x, x, u) \in\left(\left.p_{0}^{(1)}\right|_{\Delta_{X}}\right)^{*} E$. Then, $\omega \in S F_{\text {inv }}\left(\pi, \Delta_{X}\right)$. Let $\pi$ be a principal $G$-bundle. Then $\omega \in S F_{\text {inv }}\left(\pi, \Delta_{X}\right)_{G}$ and we can see that this $\omega$ essentially coincides with the translation function.

The following is a slight generalization of an example in [13].

ExAmple 3.3. Let $X=\mathbf{R}, G=\mathbf{R}^{\times}$and $\mathrm{pr}_{1}: \mathbf{R} \times \mathbf{R}^{\times} \rightarrow \mathbf{R}$ be the product bundle, where $\mathbf{R}^{\times}:=\mathbf{R} \backslash\{0\}$ is a topological group with respect to the multiplication. Let $U \subset \mathbf{R}^{2}$ with $\Delta_{\mathbf{R}} \subset U$ and $f: U \rightarrow \mathbf{R}$ be a continuous map such that $f(x, x)=0$ for $x \in \mathbf{R}$. Put

$$
\omega(x, y,(y, a)):=\left(x, a e^{f(x, y)}\right)
$$

for $(x, y,(y, a)) \in\left(\left.p_{0}^{(1)}\right|_{U}\right)^{*}\left(\mathbf{R} \times \mathbf{R}^{\times}\right)$. Then $\omega \in S F\left(\mathrm{pr}_{1}, U\right)_{\mathbf{R}^{\times}}$. If $f$ satisfies the condition $f(x, y)=-f(y, x)$ for $(x, y) \in U$, then $\omega$ is invertible.

On a principal $G$-bundle satisfying appropriate condition, there exists a slicing function, which corresponds to a flat connection in the smooth category.

Example 3.4. Let $\pi: E \rightarrow X$ be a locally trivial $G$-bundle and $A$ a bundle atlas (a system of local trivializations). Suppose that $G$ is a discrete group and for any $(\alpha, \beta) \in A^{2}, U_{\alpha} \cap U_{\beta}$ is connected. Put $U_{A}:=\bigcup_{\alpha \in A} U_{\alpha} \times U_{\alpha}$. For $(x, y, u) \in$ $\left(\left.p_{0}^{(1)}\right|_{U_{A}}\right)^{*} E$, if $(x, y) \in U_{\alpha} \times U_{\alpha}$, put

$$
\omega_{A}(x, y, u):=\alpha^{-1}\left(x,\left(\operatorname{pr}_{2} \circ \alpha\right)(u)\right) .
$$

This definition does not depend on the choice of $\alpha$. In fact, for any $\beta \in A$ and $(x, y, u) \in\left(\left.p_{0}^{(1)}\right|_{U_{A}}\right)^{*} E$, we have

$$
\begin{aligned}
\beta^{-1}\left(x,\left(\operatorname{pr}_{2} \circ \beta\right)(u)\right) & =\alpha^{-1}\left(x,\left(\operatorname{pr}_{2} \circ \alpha \circ \beta^{-1}\right)\left(x,\left(\operatorname{pr}_{2} \circ \beta \circ \alpha^{-1}\right)\left(y,\left(\operatorname{pr}_{2} \circ \alpha\right)(u)\right)\right)\right) \\
& \left.\left.=\alpha^{-1}\left(x, g_{\alpha \beta}(x) g_{\beta \alpha}(y)\left(\operatorname{pr}_{2} \circ \alpha\right)(u)\right)\right)\right)=\alpha^{-1}\left(x,\left(\operatorname{pr}_{2} \circ \alpha\right)(u)\right) .
\end{aligned}
$$

We can see that $\omega_{A} \in S F_{\text {inv }}\left(\pi, U_{A}\right)_{G}$. By the definition, we have

$$
\left(\omega_{A}\right)_{x, y} \circ\left(\omega_{A}\right)_{y, z}=\left(\omega_{A}\right)_{x, z}
$$

for any $\alpha \in A$ and $x, y, z \in U_{\alpha}$. 
In the following example, we shall review the universal bundle constructed by Milnor and demonstrate a slicing function which plays an important role in the proof of Theorem 5.1 in [14].

EXAmple $3.5([14])$. Let $X$ be a polyhedron of a countable connected simplicial complex $K$ in the weak topology. Put $U_{K}:=\bigcup_{\tau \in K}|\tau| \times|\tau|, \quad X^{\sqcup}:=$ $\bigcup_{n \geq 0} X^{n+1}$ (topological sum), and

$$
\Im_{K}:=\left\{\left(x_{n}, \ldots, x_{0}\right) \in X^{\sqcup} \mid n \geq 1 \Rightarrow \forall i \in\{1, \ldots, n\}:\left(x_{i}, x_{i-1}\right) \in U_{K}\right\} .
$$

An equivalence relation in $\mathfrak{S}_{K}$ is generated by the relations

$$
\left(x_{n}, \ldots, x_{i}, \ldots, x_{0}\right) \sim\left(x_{n}, \ldots, \hat{x}_{i}, \ldots, x_{0}\right)
$$

whenever either $x_{i}=x_{i-1}$ or $x_{i+1}=x_{i-1}$, where the symbol $\hat{x}$ denotes deletion of $x$. We denote by $\left[x_{n} \ldots, x_{0}\right]$ the equivalence class of $\left(x_{n}, \ldots, x_{0}\right)$. Fix a vertex $v_{0}$ of $K$. Put

$$
\begin{aligned}
& \tilde{\mathfrak{\Xi}}_{K}:=\mathfrak{\Im}_{K} / \sim, \\
& \tilde{E}_{K}:=\left\{\left[x_{n}, \ldots, x_{1}, x_{0}\right] \in \tilde{\mathfrak{\Xi}}_{K} \mid x_{0}=v_{0}\right\}, \\
& \tilde{G}_{K}:=\left\{\left[x_{n}, \ldots, x_{1}, v_{0}\right] \in \tilde{E}_{K} \mid x_{n}=v_{0}\right\},
\end{aligned}
$$

where a topology of $\tilde{\mathfrak{S}}_{K}$ is the quotient topology and consider $\tilde{E}_{K}$ and $\tilde{G}_{K}$ as subspaces. A unary operation $\cdot^{-}$on $\tilde{\mathfrak{\Xi}}_{K}$ is defined by

$$
\left[x_{n}, \ldots, x_{1}, x_{0}\right]^{-}:=\left[x_{0}, x_{1}, \ldots, x_{n}\right]
$$

for $\left[x_{n}, \ldots, x_{1}, x_{0}\right] \in \tilde{\mathfrak{\Xi}}_{K}$. A partial binary operation on $\tilde{\mathfrak{\Xi}}_{K}$ is defined by

$$
\left[x_{n}, \ldots, x_{0}\right]\left[y_{m}, \ldots, y_{0}\right]:=\left[x_{n}, \ldots, x_{0}, y_{m}, \ldots, y_{0}\right]
$$

for $\left(\left[x_{n}, \ldots, x_{0}\right],\left[y_{m}, \ldots, y_{0}\right]\right) \in \tilde{\Xi}_{K} \times \tilde{\Xi}_{K}$ such that $x_{0}=y_{m}$. We can see that $\tilde{G}_{K}$ is a topological group with respect to these operations. A bundle $\tilde{\pi}_{K}: \tilde{E}_{K} \rightarrow X$ is defined by

$$
\tilde{\pi}_{K}\left(\left[x_{n}, \ldots, x_{1}, v_{0}\right]\right):=x_{n} .
$$

We can see that $\tilde{\pi}_{K}$ is a locally trivial principal $\tilde{G}_{K}$-bundle and a universal bundle, that is, $\tilde{E}_{K}$ is $\infty$-connected. For $\left(x, y_{m},\left[y_{m}, \ldots, y_{1}, v_{0}\right]\right) \in\left(\left.p_{0}^{(1)}\right|_{U}\right)^{*} \tilde{E}_{K}$, put

$$
\tilde{\omega}_{K}\left(x, y_{m},\left[y_{m}, \ldots, y_{1}, v_{0}\right]\right):=\left[x, y_{m}\right]\left[y_{m}, \ldots, y_{1}, v_{0}\right] .
$$

Then $\tilde{\omega}_{K} \in S F_{\text {inv }}\left(\tilde{\pi}_{K}, U\right)_{\tilde{G}_{K}}$. 
In Section 8 , we will see that $\tilde{\pi}_{K}$ together with the parallel displacement induced by $\tilde{\omega}_{K}$ is an initial object in a category of principal bundles with parallel displacements over $X$.

EXAmple $3.6([14])$. Let $X$ be a polyhedron of a countable simplicial complex $K$ in the weak topology and $\pi: E \rightarrow X$ a locally trivial $G$-bundle. Using an obstruction argument, Milnor proves that there exists $\omega \in S F_{\text {inv }}\left(\pi, U_{K}\right)_{G}$, where $U_{K}=\bigcup_{\tau \in K}|\tau| \times|\tau|$.

The next example and the succeeding arguments indicate that the slicing function is a generalization of the connection in the smooth category.

ExAmple 3.7. Let $\pi: E \rightarrow X$ be a smooth principal $G$-bundle, where $G$ is a Lie group. A connection (invariant horizontal subbundle) in $\pi$ is a smooth subbundle $H$ of $T E$ such that

(1) $T_{u} E=\operatorname{Ker} \pi_{* u} \oplus H_{u}$ for $u \in E$,

(2) $r_{a * u}\left(H_{u}\right)=H_{u a}$ for $(u, a) \in E \times G$,

where $\pi_{*}$ is the differential of $\pi$ (for example, [12]) and $r_{a}: E \rightarrow E$ is given by $r_{a}(v):=v a$ (see Section 2). Let $H$ be a connection and $c:[0,1] \rightarrow X$ a piecewise smooth curve. For $u \in E$, there exists a unique curve $\tilde{c}: I \rightarrow E$ such that $\tilde{c}(0)=u$, $\pi \circ \tilde{c}=c$, and $\frac{d \tilde{c}}{d t}(t) \in H_{\tilde{c}(t)}$ for $t \in I$, that is, $\tilde{c}$ is the horizontal lift of $c$ starting from $u$. Let $X$ be a Riemannian manifold. A subset $V$ in $X$ is strongly convex if for any $(x, y) \in V \times V$ there exists a unique geodesic in $V$ joining $y$ to $x$, and such that the length of the geodesic is the distance $d(x, y)$, where the geodesic is $\gamma(t)=\exp _{y} t v$ such that $\gamma(1)=x$. Fix an open covering $\mathscr{V}$ of $X$ which consists of strongly convex sets. Put $U_{\mathscr{V}}:=\bigcup_{V \in \mathscr{V}} V \times V$. Let $(x, y, u) \in\left(\left.p_{0}^{(1)}\right|_{U_{\mathscr{\gamma}}}\right)^{*} E$ and $\gamma$ be the geodesic in some $V$ joining $y$ to $x$. Let $\tilde{\gamma}$ be the horizontal lift of $\gamma$ starting from $u$. Put

$$
\omega^{H}(x, y, u):=\tilde{\gamma}(1)
$$

Then we can see that $\omega^{H} \in S F_{\text {inv }}\left(\pi, U_{\mathscr{V}}\right)_{G}$ and it is smooth.

In $[13,16]$, Kubarski and Teleman introduce the notion of direct connections, which are in fact the smooth slicing functions in smooth vector bundles. They show that the Chern character of smooth vector bundles can be represented as 
the periodic cyclic homology class of a specific cyclic cycle, manufactured from a direct connection. Let $K \in\{\mathbf{R}, \mathbf{C}\}, \pi: E \rightarrow X$ be a smooth $K$-vector bundle, $U \subset X^{2}$ an open neighborhood of $\Delta_{X}$, and $G L(E):=\bigcup_{(x, y) \in X^{2}} \operatorname{Iso}_{K}\left(E_{y}, E_{x}\right)$. Note that $G L(E)$ is a smooth fiber bundle with standard fiber $G L\left(K^{n}\right)$. By a linear direct connection in $E$ they mean a smooth map $\tau: U \rightarrow G L(E)$ such that

(1) $\tau(x, y): E_{y} \rightarrow E_{x}$ for $(x, y) \in U$,

(2) $\tau(x, x)=i d_{E_{x}}$ for $x \in X$.

Let $D C_{K}(\pi, U)$ denote the set of linear direct connections. Let $L E:=$ $\bigcup_{x \in X} \operatorname{Iso}_{K}\left(K^{n}, E_{x}\right)$ be the linear frame bundle and $\pi_{L E}$ its projection. For $\tau \in D C_{K}(\pi, U)$, put

$$
\omega^{\tau}(x, y, u):=\tau(x, y) \circ u
$$

for $(x, y, u) \in\left(\left.p_{0}^{(1)}\right|_{U}\right)^{*} L E$. Then we can see that $\omega^{\tau} \in S F\left(\pi_{L E}, U\right)_{G L\left(K^{n}\right)}$. Conversely, for $\omega \in S F\left(\pi_{L E}, U\right)_{G L\left(K^{n}\right)}$, put

$$
\tau^{\omega}(x, y):=\omega(x, y, u) \circ u^{-1}
$$

for $(x, y) \in U$ and $u \in \operatorname{Iso}_{K}\left(K^{n}, E_{y}\right)$. Then we can see that this definition does not depend on the choice of $u$ and that $\tau^{\omega} \in D C_{K}(\pi, U)$. Since $\tau^{\omega^{\tau}}=\tau$ and $\omega^{\tau^{\omega}}=\omega$ for any $\tau \in D C_{K}(\pi, U)$ and $\omega \in S F\left(\pi_{L E}, U\right)_{G L\left(K^{n}\right)}$, an element of $D C_{K}(\pi, U)$ corresponds bijectively to the element of $S F\left(\pi_{L E}, U\right)_{G L\left(K^{n}\right)}$.

In [13], they remark that the parallel transport along small geodesicsdefined for a linear connection $\nabla$ in $E$-produces a direct connection $\tau^{\nabla}$ in $E$. Their construction is a special case of that in Example 3.7.

REMARK 3.2. In [13], Kubarski and Teleman assume that the base manifold is endowed with an affine connection. In that situation, we can also construct smooth slicing function $\omega^{H}$ by taking a similar covering as in Example 3.7.

On the other hand, Kubarski and Teleman show that a linear direct connection $\tau$ induces a linear connection $\nabla^{\tau}$ in $E$. We generalize their construction to a smooth principal $G$-bundle.

Proposition 3.1. Let $\pi: E \rightarrow X$ be a smooth principal $G$-bundle, $U \subset X^{2}$ an open neighborhood of $\Delta_{X}$ and $\omega \in S F(\pi, U)_{G}$ a smooth map. For $u \in E$, put

$$
H_{u}^{\omega}:=\left\{\omega(\cdot, \pi(u), u)_{* \pi(u)}(v) \mid v \in T_{\pi(u)} X\right\}
$$


where $\omega(\cdot, \pi(u), u): p_{1}((X \times\{\pi(u)\}) \cap U) \rightarrow E$ is a map such that $\pi \circ(\omega(\cdot, \pi(u), u))$ $=i d_{p_{1}((X \times\{\pi(u)\}) \cap U)}$. Then $H^{\omega}:=\bigcup_{u \in E} H_{u}^{\omega}$ is a connection in $E$, and if $\left[\omega_{\mathcal{O}_{X^{2}}\left(\Delta_{X}\right)}\right.$ $=\left[\omega^{\prime}\right]_{\mathcal{O}_{X^{2}}\left(\Delta_{X}\right)} \in \lim _{\longrightarrow} S F(\pi, U)_{G}$, then $H^{\omega}=H^{\omega^{\prime}}$. Moreover, if $X$ is a Riemannian manifold, then $H^{\omega^{H}}=H$ for any connection $H$ in $E$.

Proof. We can see that $H^{\omega}$ is a smooth subbundle of TE. Since $\pi_{* u} \circ\left(\omega(\cdot, \pi(u), u)_{* \pi(u)}\right)=i d_{T_{\pi(u)} X}$, we have $\operatorname{Ker} \pi_{* u} \cap H_{u}^{\omega}=\{0\}$. For $W \in T_{u} E$, since

$$
\pi_{* u}\left(W-\omega(\cdot, \pi(u), u)_{* \pi(u)}\left(\pi_{* u}(W)\right)\right)=\pi_{* u}(W)-\pi_{* u}(W)=0,
$$

we get $W-\omega(\cdot, \pi(u), u)_{* \pi(u)}\left(\pi_{* u}(W)\right) \in \operatorname{Ker} \pi_{* u}$, and consequently $T_{u} E=\operatorname{Ker} \pi_{* u}$ $\oplus H_{u}^{\omega}$. For $a \in G$, the equality

$$
r_{a * u} \circ \omega(\cdot, \pi(u), u)_{* \pi(u)}=\omega(\cdot, \pi(u a), u a)_{* \pi(u a)}
$$

implies $r_{a * u}\left(H_{u}\right)=H_{u a}^{\omega}$. Thus, $H^{\omega}$ is a connection.

Let $\mathscr{D}$ be the maximal domain of the exponential map, $u \in E$, and $v \in$ $\mathscr{D} \cap T_{\pi(u)} X$. Put $\gamma_{v}(t):=\exp _{\pi(u)} t v$ for $t \in[0,1]$ and let $\tilde{\gamma}$ be the horizontal lift of $\gamma$ starting from $u$. From the equality $\gamma_{t v}(s)=\gamma_{v}(s t)$ and the uniqueness of the horizontal lift, we have $\tilde{\gamma}_{t v}(s)=\tilde{\gamma}_{v}(s t)$. Then we get

$$
\omega^{H}\left(\gamma_{v}(t), \pi(u), u\right)=\omega^{H}\left(\gamma_{t v}(1), \pi(u), u\right)=\tilde{\gamma}_{t v}(1)=\tilde{\gamma}_{v}(t) .
$$

Thus $\omega^{H}(\cdot, \pi(u), u)_{* \pi(u)}(v)=\dot{\tilde{\gamma}}_{v}(0) \in H_{u}$, and consequently $H_{u}^{\omega^{H}} \subset H_{u}$. Since $\operatorname{dim} H_{u}^{\omega^{H}}=\operatorname{dim} H_{u}$, we have $H_{u}^{\omega^{H}}=H_{u}$.

Note that a smooth slicing function $\omega$ which is not necessarily invertible induces a connection $H^{\omega}$ while a smooth slicing function $\omega^{H}$ derived from a connection $H$ is invertible.

In the following example, we give concrete expressions of $H^{\omega}$ and $\omega^{H^{\omega}}$ for $\omega$ in Example 3.3.

Example 3.8. Let $f: U \rightarrow \mathbf{R}$ and $\omega \in S F\left(\mathrm{pr}_{1}, U\right)_{\mathbf{R}^{\times}}$as in Example 3.3. Suppose that $U$ is an open set and $\partial_{1} f$ exists and is smooth on $\Delta_{\mathbf{R}}$. For $(y, a) \in \mathbf{R} \times \mathbf{R}^{\times}$, since

$$
\omega(\cdot, y,(y, a))_{* y}\left(\left(\partial_{1}\right)_{y}\right)=\left(\partial_{1}\right)_{(y, a)}+a \partial_{1} f(y, y)\left(\partial_{2}\right)_{(y, a)},
$$

we have

$$
H_{(y, a)}^{\omega}=\left\{c\left(\partial_{1}\right)_{(y, a)}+c a \partial_{1} f(y, y)\left(\partial_{2}\right)_{(y, a)} \mid c \in \mathbf{R}\right\}
$$


Let $(x, y) \in \mathbf{R} \times \mathbf{R}$ and $\gamma(t)=(x-y) t+y$ be the geodesic joining $y$ to $x$. Then horizontal lift $\xi$ of $\gamma$ starting from $(y, a) \in \mathbf{R} \times \mathbf{R}^{\times}$is given by

$$
\xi(t)=\left((x-y) t+y, a e^{(x-y) \int_{0}^{t} \partial_{1} f((x-y) s+y,(x-y) s+y) d s}\right) .
$$

Thus, $\omega^{H^{\omega}} \in S F_{\text {inv }}\left(\mathrm{pr}_{1}, \mathbf{R} \times \mathbf{R}\right)_{\mathbf{R}^{\times}}$is given by

$$
\omega^{H^{\omega}}(x, y,(y, a))=\left(x, a e^{\int_{y}^{x} \partial_{1} f(t, t) d t}\right) .
$$

From this expression, it follows that $\left[\omega^{H^{\omega}}\right]_{\mathcal{O}_{\mathbf{R}^{2}}\left(\Delta_{\mathbf{R}}\right)}=[\omega]_{\mathcal{R}_{\mathbf{R}^{2}}\left(\Delta_{\mathbf{R}}\right)} \in \lim _{\longrightarrow} S F\left(\operatorname{pr}_{1}, U\right)_{\mathbf{R}^{\times}}$ if and only if $f(x, y)=\int_{y}^{x} \partial_{1} f(t, t) d t$ for some open neighborhood $V \subset U$ of $\Delta_{\mathbf{R}}$ and any $(x, y) \in V$.

A connection in the sense of Asada is a germ of slicing functions over neighborhoods of the diagonal set of the base space of a $G$-bundle.

Example 3.9 (cf. $[3,4,5]$ ). Let $\pi: E \rightarrow X$ be a $G$-bundle and $U \subset X^{2}$ such that $\Delta_{X} \subset U$. We denote by $C^{1}(\pi, U)_{G}$ the set of continuous maps $s: E^{2}\left\lceil_{U} \rightarrow G\right.$ such that

(1) $s(u, u)=1_{G}$ for $u \in E$,

(2) $s(v a, u b)=a^{-1} s(v, u) b$ for $(v, u) \in E^{2}\left\lceil_{U}\right.$ and $a, b \in G$.

Regarding an element $[s]_{\mathscr{N}_{X^{2}}\left(\Delta_{X}\right)} \in \lim _{\longrightarrow} C^{1}(\pi, U)_{G}$ as a connection in $\pi$, Asada generalizes connection theory to a category of topological fiber bundles, where $\mathscr{N}_{X^{2}}\left(\Delta_{X}\right)$ is the set of neighborhoods of $\Delta_{X}$ in $X^{2}$. For $s \in C^{1}(\pi, U)_{G}$, a map $\omega^{s}:\left(\left.p_{0}^{(1)}\right|_{U}\right)^{*} E \rightarrow E$ is given by

$$
\omega^{s}(x, y, u):=v s(v, u)
$$

for $(x, y, u) \in\left(\left.p_{0}^{(1)}\right|_{U}\right)^{*} E$, where $v \in E_{x}$. We can see that this definition does not depend on the choice of $v$ and that $\omega^{s} \in S F(\pi, U)_{G}$. Suppose that $\pi$ is a principal $G$-bundle. For $\omega \in S F(\pi, U)_{G}$, a map $s^{\omega}: E^{2}\left\lceil_{U} \rightarrow G\right.$ is given by

$$
s^{\omega}(v, u):=T(v, \omega(\pi(v), \pi(u), u))
$$

for $(v, u) \in E^{2}\left\lceil_{U}\right.$. By the definition, $s^{\omega} \in C^{1}(\pi, U)_{G}$. We can see that $s^{\omega^{s}}=s$ and $\omega^{s^{\omega}}=\omega$ for any $s \in C^{1}(\pi, U)_{G}$ and $\omega \in S F(\pi, U)_{G}$. Let $U$ be symmetric and $C_{\text {inv }}^{1}(\pi, U)_{G}$ denote the set of $s \in C^{1}(\pi, U)_{G}$ such that $s(u, v)=s(v, u)^{-1}$ for $(v, u) \in E^{2}\left\lceil_{U}\right.$. Then $C_{\text {inv }}^{1}(\pi, U)_{G}$ corresponds to $S F_{\text {inv }}(\pi, U)_{G}$. 


\section{Parallel Displacements along Admissible Sequences}

In this section, we introduce parallel displacements along admissible sequences. First of all, we introduce admissible sequence spaces, which is topologized so that operations are continuous.

Let $X$ be a topological space and $X^{\sqcup}:=\bigcup_{n \geq 0} X^{n+1}$ the topological sum. Two maps $p_{0}, p_{\infty}: X^{\sqcup} \rightarrow X$ are defined by

$$
p_{0}\left(x_{n}, \ldots, x_{0}\right)=x_{0}, \quad p_{\infty}\left(x_{n}, \ldots, x_{0}\right)=x_{n}
$$

for $\left(x_{n}, \ldots, x_{0}\right) \in X^{\sqcup}$. A binary operation • on $X^{\sqcup}$ is defined by

$$
\bullet(\mathbf{x}, \mathbf{y}):=\mathbf{x} \bullet \mathbf{y}:=\left(x_{n}, \ldots, x_{1}, y_{m}, \ldots, y_{0}\right)
$$

for $(\mathbf{x}, \mathbf{y})=\left(\left(x_{n}, \ldots, x_{0}\right),\left(y_{m}, \ldots, y_{0}\right)\right) \in X^{\sqcup} \times X^{\sqcup}$. Let $X^{\sqcup} \times_{X} X^{\sqcup}$ be a fiber product of $X^{\sqcup} \stackrel{p_{0}}{\rightarrow} X \stackrel{p_{\infty}}{\leftarrow} X^{\sqcup}$. Hereafter in this paper, we denote by the same symbol • the restriction of the binary operation $\bullet$ to $X^{\sqcup}{ }{ }_{X} X^{\sqcup}$, which is a partial binary operation on $X^{\sqcup}$. A unary operation .- ${ }^{-}$on $X^{\sqcup}$ is defined by $\mathbf{x}^{-}:=\left(x_{0}, x_{1}, \ldots, x_{n}\right)$ for $\mathbf{x}=\left(x_{n}, \ldots, x_{1}, x_{0}\right)$. For $\mathbf{x} \in X^{\sqcup}$, we say that the length of $\mathbf{x}$ is $n$ if $\mathbf{x} \in X^{n+1}$. For any subset $\subseteq \subset X^{\sqcup}$ and $n \geq 0$, put $\mathfrak{\Xi}_{(n)}:=\subseteq \cap X^{n+1}$. Note that for $\subseteq \subset X^{\sqcup}, \mathfrak{\subseteq}=\bigcup_{n \geq 0} \Im_{(n)}$. We can see that $\left(X^{\sqcup}, \bullet\right)$ is associative and generated by $X \cup X^{2}$, and maps $p_{0}, p_{\infty}, \bullet$, and $\cdot^{-}$are all continuous.

DeFINITION 4.1. We call a subspace $\subseteq \subset X^{\sqcup}$ an admissible sequence space over $X$ if it satisfies the following conditions:

(a) $\bullet\left(\mathfrak{\Im}^{2} \cap\left(X^{\sqcup} \times_{X} X^{\sqcup}\right)\right) \subset \mathfrak{s}$.

(b) $X \cup \Delta_{X} \subset \Im$.

(c) $\left\{\mathbf{x}^{-} \mid \mathbf{x} \in \Xi\right\} \subset \Xi$.

We denote by $\mathscr{A} \mathscr{S}(X)$ the set of admissible sequence spaces over $X$. Let $\mathfrak{\Xi} \in \mathscr{A} \mathscr{S}(X)$. We say that $X$ is $\mathfrak{\Xi}$-connected if $\left(p_{\infty} \hat{\times} p_{0}\right)(\mathfrak{\Xi})=X^{2}$, that is, for any $(x, y) \in X^{2}$, there exists $\mathbf{x} \in \mathfrak{S}$ such that $p_{0}(\mathbf{x})=y$ and $p_{\infty}(\mathbf{x})=x$. For a subset $A \subset X^{2}$, put

$$
\Xi_{A}:=\left(p_{\infty}\left|\Subset \hat{\times} p_{0}\right|_{\Xi}\right)^{-1}(A)
$$

For $(x, y) \in X^{2}$, put $\mathfrak{\Xi}_{x, y}:=\mathfrak{\Xi}_{\{(x, y)\}}$ and $\mathfrak{\Xi}_{x}:=\mathfrak{\Xi}_{x, x}$. For a symmetric subspace $U \subset X^{2}$ such that $\Delta_{X} \subset U$, put

$$
\langle U\rangle:=\left\{\left(x_{n}, \ldots, x_{0}\right) \in X^{\sqcup} \mid n \geq 1 \Rightarrow \forall i \in\{1, \ldots, n\}:\left(x_{i}, x_{i-1}\right) \in U\right\} .
$$


Note that for $x \in X, \mathfrak{\Xi}_{X \times\{x\}}=\left(\left.p_{0}\right|_{\mathfrak{S}}\right)^{-1}(\{x\})$ and $\mathfrak{\Xi}_{\{x\} \times X}=\left(\left.p_{\infty}\right|_{\mathfrak{S}}\right)^{-1}(\{x\})$. The condition (a) implies $\left\langle\mathfrak{S}_{(1)}\right\rangle \subset \mathfrak{G}$.

DeFinition 4.2. Put

$$
\mathscr{S}_{X^{2}}\left(\Delta_{X}\right):=\left\{U \subset X^{2} \mid \forall x \in X, \exists V \in \mathcal{O}_{X}(x): V \times\{x\} \subset U\right\},
$$

where $\mathcal{O}_{X}(x)$ is the set of all open neighborhoods of $x$.

The following example of admissible sequence space is typical.

Example 4.1 (cf. [14]). Let $X$ be a polyhedron of a countable simplicial complex $K$ in the weak topology. As we have already seen in Examples 3.5 and 3.6, put $U_{K}:=\bigcup_{\tau \in K}|\tau| \times|\tau|$. Then, $\Delta_{X} \subset U_{K} \subset X^{2}$ and $\left\langle U_{K}\right\rangle \in \mathscr{A} \mathscr{S}(X)$. Note that $\mathfrak{\Xi}_{K}=\left\langle U_{K}\right\rangle$. For $x \in X$, let $V_{K}(x)$ be the open star neighborhood of $x$ in $X$. Then, $V_{K}(x) \times\{x\} \subset U_{K}$ and $\left\langle U_{K}\right\rangle_{(1)}=U_{K} \in \mathscr{S}_{X^{2}}\left(\Delta_{X}\right)$. If $K$ is connected as simplicial complex, then $X$ is $\left\langle U_{K}\right\rangle$-connected.

We have a sufficient condition for a pathwise connected space to be $\mathfrak{\Xi}$ connected as follows.

Proposition 4.1. Let $X$ be pathwise connected and $\mathfrak{\Xi} \in \mathscr{A} \mathscr{S}(X)$ with $\mathfrak{\Xi}_{(1)} \in$ $\mathscr{S}_{X^{2}}\left(\Delta_{X}\right)$. Then, $X$ is $\left\langle\mathfrak{S}_{(1)}\right\rangle$-connected, and consequently $\mathfrak{S}_{\text {-connected. }}$

Proof. Let $(x, y) \in X \times X$. Since $X$ is pathwise connected, there exists a curve $c:[0,1] \rightarrow X$ such that $c(0)=y$ and $c(1)=x$. For $z \in X$, fix $V_{z} \in \mathcal{O}_{X}(z)$ such that $V_{z} \times\{z\} \subset \mathfrak{S}_{(1)}$. Then, $\left(c^{-1}\left(V_{z}\right)\right)_{z \in X}$ is a covering of $[0,1]$. Let $\varepsilon>0$ be a Lebesgue number of $\left(c^{-1}\left(V_{z}\right)\right)_{z \in X}$. Let $\left(t_{i}\right)_{i \in\{0, \ldots, k\}}$ be a partition of $[0,1]$ such that $0=t_{0}<t_{1}<\cdots<t_{k}=1$ and $t_{i}-t_{i-1}<\varepsilon$ for $i \in\{1, \ldots, k\}$. For $i \in\{1, \ldots, k\}$, put $w_{i}:=c\left(t_{i}\right)$ and fix $V_{z_{i}}$ such that $c\left(\left[t_{i-1}, t_{i}\right]\right) \subset V_{z_{i}}$. Then, $\left(z_{i}, w_{i-1}\right) \in\left\{z_{i}\right\} \times V_{z_{i}} \subset \mathfrak{S}_{(1)}$ for any $i \in\{2, \ldots, k\}$ and $\left(w_{i}, z_{i}\right) \in V_{z_{i}} \times\left\{z_{i}\right\} \subset \mathfrak{S}_{(1)}$ for any $i \in\{1, \ldots, k\}$. Therefore,

$$
\left(x, z_{k}, w_{k-1}, \ldots, w_{1}, z_{1}, y\right) \in\left\langle\Im_{(1)}\right\rangle \subset \Subset
$$

and this completes the proof.

We prepare the following proposition which will not be needed until the proof of Theorem 6.3 (Holonomy Reduction Theorem) but being related only to admissible sequences. 
Proposition 4.2. For $\mathfrak{S} \in \mathscr{A} \mathscr{S}(X)$, we have the following:

(i) $\left.p_{\infty}\right|_{\mathfrak{S}}: \mathfrak{S} \rightarrow X$ is an identification.

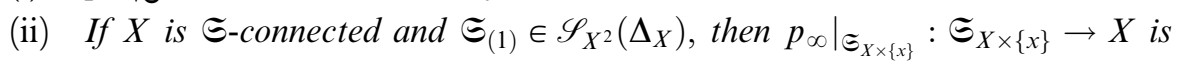
an identification for any $x \in X$.

PROOF. Note that $\left.p_{\infty}\right|_{\Xi_{(n)}}=\left.p_{n}^{(n)}\right|_{\Xi_{(n)}}$ for $n \geq 0$, where $p_{n}^{(n)}: X^{n+1} \rightarrow X$ is the projection such that $p_{n}^{(n)}\left(x_{n}, \ldots, x_{0}\right)=x_{n}$.


Since

$$
\left(p_{\infty} \mid \varsigma^{-1}(V)=\bigcup_{n \geq 0}\left(\left.p_{n}^{(n)}\right|_{\varsigma_{(n)}}\right)^{-1}(V),\right.
$$

$\left(\left.p_{n}^{(n)}\right|_{\Xi_{(n)}}\right)^{-1}(V)$ is an open set in $\mathfrak{\Xi}_{(n)}$ for any $n \geq 0$. Equalities $\mathfrak{\Xi}_{(0)}=X$ and $p_{0}^{(0)}=i d_{X}$ imply that $V=\left(p_{0}^{(0)}\right)^{-1}(V)$ is an open set in $X$, and we obtain (i).

Secondly, we show (ii). Let $V \subset X$ such that $\left(\left.p_{\infty}\right|_{\varsigma_{X \times\{x\}}}\right)^{-1}(V)$ is an open set in $\mathfrak{\Xi}_{X \times\{x\}}$. Since

$$
\left(\left.p_{\infty}\right|_{\mathfrak{\Xi}_{X \times\{x\}}}\right)^{-1}(V)=\bigcup_{m \geq 0}\left(\left.p_{m}^{(m)}\right|_{\left(\Im_{X \times\{x\}}\right)_{(m)}}\right)^{-1}(V),
$$

$\left(\left.p_{m}^{(m)}\right|_{\left(\Im_{X \times\{x\}}\right)_{(m)}}\right)^{-1}(V)$ is an open set in $\left(\widetilde{\Xi}_{X \times\{x\}}\right)_{(m)}$ for any $m \geq 0$. Note that

$$
\left(\left.p_{m}^{(m)}\right|_{\left(\Im_{X \times\{x\}}\right)_{(m)}}\right)^{-1}(V)=\Im_{(m)} \cap\left(V \times X^{m-1} \times\{x\}\right)
$$

for any $m \geq 1$ and $\left(\left.p_{0}^{(0)}\right|_{\left(\Im_{X \times\{x\}}\right)_{(0)}}\right)^{-1}(V)=V \cap\{x\}$. Let $y \in V$ and $\left(y, x_{n-1}, \ldots\right.$, $\left.x_{1}, x\right) \in \mathfrak{\Xi}_{y, x}$. Then

$$
\left(y, y, x_{n-1}, \ldots, x\right)=(y, y) \bullet\left(y, x_{n-1}, \ldots, x\right) \in\left(\left.p_{n+1}^{(n+1)}\right|_{\left(\Im_{X \times\{x\}}\right)_{(n+1)}}\right)^{-1}(V) .
$$

Let $V^{\prime}$ be an open neighborhood of $y$ in $X$ such that $V^{\prime} \times\{y\} \subset \Xi_{(1)}$. Since - $\left(\mathfrak{S}_{(1)} \times{ }_{X} \mathfrak{\Xi}_{(n)}\right) \subset \mathfrak{S}_{(n+1)}$, we have $V^{\prime} \times\{y\} \times\left\{x_{n-1}\right\} \times \cdots \times\{x\} \subset \mathfrak{\Xi}_{(n+1)} \cap$ $\left(X^{n+1} \times\{x\}\right)=\left(\Im_{X \times\{x\}}\right)_{(n+1)}$. On the other hand, since $\left(\left.p_{n+1}^{(n+1)}\right|_{\left(\subseteq_{X \times\{x\}}\right)_{(n+1)}}\right)^{-1}(V)$ is an open set in $\left(\Xi_{X \times\{x\}}\right)_{(n+1)}$, there exists an open set $W$ in $X^{n+2}$ such that

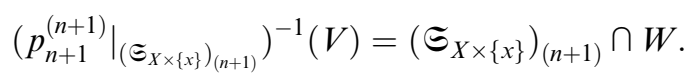

Then for $i \in\{0, \ldots, n+1\}$, there exists an open neighborhood $W_{i}$ of $x_{i}$ in $X$ such that $W_{n+1} \times \cdots \times W_{0} \subset W$, where $x_{0}=x$ and $x_{n+1}=x_{n}=y$. Thus

$$
\begin{aligned}
\left(V^{\prime} \cap W_{n+1}\right) \times\{y\} \times\left\{x_{n-1}\right\} \times \cdots \times\{x\} & \subset\left(\Im_{X \times\{x\}}\right)_{(n+1)} \cap W \\
& =\left(\left.p_{n+1}^{(n+1)}\right|_{\left(\mathfrak{\Xi}_{X \times\{x\}}\right)_{(n+1)}}\right)^{-1}(V) .
\end{aligned}
$$


Therefore, $\quad\left(V^{\prime} \cap W_{n+1}\right) \times\{y\} \times\left\{x_{n-1}\right\} \times \cdots \times\{x\} \subset V \times X^{n} \times\{x\}$. Thus $y \in$ $V^{\prime} \cap W_{n+1} \subset V$ and $V$ is an open set in $X$.

Next we introduce parallel displacements along admissible sequences. Hereafter in this section, let $\pi: E \rightarrow X$ be a bundle and $\subseteq \in \mathscr{A} \mathscr{S}(X)$.

Definition 4.3. Let $P:\left(\left.p_{0}\right|_{\Im}\right)^{*} E \rightarrow E$ be a continuous map. Put $P_{\mathbf{x}}:=$ $P(\mathbf{x}, \cdot): E_{p_{0}(\mathbf{x})} \rightarrow E$ for $\mathbf{x} \in \mathfrak{S}$. We call $P$ a parallel displacement along $\subseteq$ in $\pi$ if it satisfies the following conditions:

(1) $\left(P,\left.p_{\infty}\right|_{\Xi}\right) \in \operatorname{Hom}\left(\left(\left.p_{0}\right|_{\Xi}\right)^{*} \pi, \pi\right)$.

(2) $P_{(x, x)}=i d_{E_{x}}$ for any $(x, x) \in \Xi_{(1)}$.

(3) $P_{\mathbf{x} \bullet \mathbf{y}}=P_{\mathbf{x}} \circ P_{\mathbf{y}}$ for any $(\mathbf{x}, \mathbf{y}) \in \mathfrak{\Xi}^{2} \cap\left(X^{\sqcup} \times_{X} X^{\sqcup}\right)$.

(4) $P_{\mathbf{x}^{-}}=P_{\mathbf{x}}^{-1}$ for any $\mathbf{x} \in \Xi$.

Let $G$ be a topological group and $\pi$ a $G$-bundle. Then, $P$ is said to be $G$ compatible if $\left(P, p_{\infty} \mid \Subset\right) \in \operatorname{Hom}_{G}\left(\left(\left(p_{0} \mid \Subset\right)^{*} \pi, G\right),(\pi, G)\right)$. We denote by $\mathscr{P} \mathscr{D}(\pi, \mathfrak{\Xi})$ (resp. $\left.\mathscr{P} \mathscr{D}(\pi, \subseteq){ }_{G}\right)$ the set of all parallel (resp. $G$-compatible) displacements along $\Im$ in $\pi$.

Note that for $x \in \mathfrak{S}_{(0)}=X, P_{x}=i d_{E_{x}}$. Suppose that there exists $P \in \mathscr{P} \mathscr{D}(\pi, \mathfrak{S})$ and $X$ is $\mathfrak{\Xi}_{\text {-connected. Let }}(x, y) \in X^{2}$ and $\mathbf{x} \in \mathfrak{\Xi}_{x, y}$. Then, from the condition (4), $P_{\mathbf{x}}: E_{y} \rightarrow E_{x}$ is a homeomorphism. Thus $\pi$ has a standard fiber. A parallel displacement is induced by a given invertible slicing function in a natural manner as follows.

Proposition 4.3 (cf. page 283 of [14]). Let $\omega \in S F_{\text {inv }}(\pi, U)$ and $\mathfrak{s}=\langle U\rangle$. Put

$$
P^{\omega}\left(\left(x_{n}, \ldots, x_{0}\right), u\right):=\left(\omega_{x_{n}, x_{n-1}} \circ \cdots \circ \omega_{x_{1}, x_{0}}\right)(u)
$$

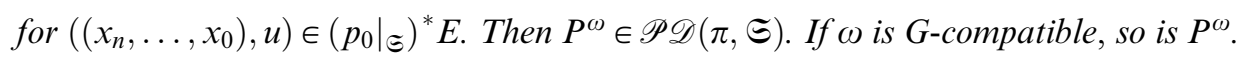

Proof. Since $\mathfrak{S}$ is the topological sum of $\mathfrak{S}_{(n)}$ with $n \geq 0$, we only need to show that $P^{\omega}$ is continuous on each $\mathfrak{\Xi}_{(n)}$. In fact, we can see that $P^{\omega}$ is written as a composition of continuous maps on each $\mathfrak{S}_{(n)}$. The $G$-compatibility of $P^{\omega}$ follows from that of $\omega$.

Let $P \in \mathscr{P} \mathscr{D}(\pi, \mathfrak{\Xi})$. For any $n \geq 0$, restricting $P$ to $\mathfrak{\Xi}_{(n)}$, we obtain a continuous map $\left.P\right|_{\left(\left.p_{0}\right|_{\tilde{\Theta}_{(n)}}\right)^{*} E}$. If $n=1$, then $\left.P\right|_{\left(\left.p_{0}\right|_{\tilde{\Theta}_{(1)}}\right)^{*} E}$ is a invertible slicing function over $\mathfrak{S}_{(1)}$. 
ExAmple 4.2. Let $\mathrm{pr}_{1}: X \times G \rightarrow X, \omega \in S F_{\text {inv }}\left(\mathrm{pr}_{1}, X \times X\right)_{G}$ be as in Example 3.1. Namely, $\omega(x, y,(y, p))=(x, p)$ for $(x, y,(y, p)) \in p_{0}^{*}(X \times G)$. Then for $\left(\left(x_{n}, \ldots, x_{0}\right),\left(x_{0}, a\right)\right) \in p_{0}^{*}(X \times G)$, we have

$$
\begin{aligned}
P^{\omega}\left(\left(x_{n}, \ldots, x_{0}\right),\left(x_{0}, a\right)\right) & =\left(\omega_{x_{n}, x_{n-1}}\left(\cdots\left(\omega_{x_{2}, x_{1}}\left(\omega_{x_{1}, x_{0}}\left(x_{0}, a\right)\right)\right) \cdots\right)\right. \\
& =\left(\omega_{x_{n}, x_{n-1}}\left(\cdots\left(\omega_{x_{2}, x_{1}}\left(x_{1}, a\right)\right) \cdots\right)=\left(x_{n}, a\right) .\right.
\end{aligned}
$$

Thus, $P^{\omega} \in \mathscr{P} \mathscr{D}\left(\mathrm{pr}_{1}, X^{\sqcup}\right)_{G}$ is a trivial one.

EXAMPLE 4.3 (cf. [14]). Let $\tilde{\pi}_{K}$ be the universal bundle which we have reviewed in Example 3.5. A map $\tilde{P}_{K}:\left(\left.p_{0}\right|_{\varsigma_{K}}\right)^{*} \tilde{E}_{K} \rightarrow \tilde{E}_{K}$ is defined by

$$
\tilde{P}_{K}\left(\left(x_{n}, \ldots, x_{1}, y_{m}\right),\left[y_{m}, \ldots, y_{1}, v_{0}\right]\right):=\left[x_{n}, \ldots, x_{1}, y_{m}\right]\left[y_{m}, \ldots, y_{1}, v_{0}\right]
$$

for $\left(\left(x_{n}, \ldots, x_{1}, y_{m}\right),\left[y_{m}, \ldots, y_{1}, v_{0}\right]\right) \in\left(\left.p_{0}\right|_{\varsigma_{K}}\right)^{*} \tilde{E}_{K}$. Then $\tilde{P}_{K} \in \mathscr{P} \mathscr{D}\left(\tilde{\pi}_{K}, \Im_{K}\right)_{\tilde{G}_{K}}$. We call $\tilde{P}_{K}$ the universal parallel displacement on $\tilde{\pi}_{K}$. Since

$$
\begin{aligned}
\tilde{P}_{K}\left(\left(x_{n}, \ldots, x_{1}, y_{m}\right),\left[y_{m}, \ldots, y_{1}, v_{0}\right]\right) & =\left[x_{n}, \ldots, x_{1}, y_{m}\right]\left[y_{m}, \ldots, y_{1}, v_{0}\right] \\
& =\left[x_{n}, x_{n-1}\right] \cdots\left[x_{1}, y_{m}\right]\left[y_{m}, \ldots, y_{1}, v_{0}\right] \\
& =\left(\widetilde{\omega_{K}} x_{n}, x_{n-1} \circ \cdots \circ \widetilde{\omega_{K}} x_{1}, y_{m}\right)\left(\left[y_{m}, \ldots, y_{1}, v_{0}\right]\right) \\
& =P^{\tilde{\omega}_{K}}\left(\left(x_{n}, \ldots, x_{1}, y_{m}\right),\left[y_{m}, \ldots, y_{1}, v_{0}\right]\right)
\end{aligned}
$$

for $\left(\left(x_{n}, \ldots, x_{0}\right),\left[x_{0}, y_{m-1}, \ldots, y_{0}\right]\right) \in\left(\left.p_{0}\right|_{\varsigma_{K}}\right)^{*} \tilde{E}_{K}$, we have $\tilde{P}_{K}=P^{\tilde{\omega}_{K}}$.

For any subset $A \subset X^{2}$, restricting $P$ to $\mathfrak{S}_{A}$, we obtain a continuous map $\left.P\right|_{\left(p_{0} \mid \Xi_{A}\right)^{*} E}$. In particular, for any $n \geq 0$, we obtain a continuous map



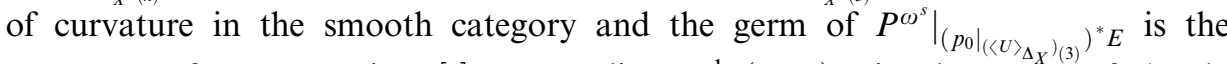
curvature of a connection $[s]_{\mathscr{N}_{X^{2}}\left(\Delta_{X}\right)} \in \lim _{\longrightarrow} C_{\text {inv }}^{1}(\pi, U)_{G}$ in the sense of Asada $[4,5]$. Thus we call $\left.P\right|_{\left(\left.p_{0}\right|_{\left(\Xi_{\left.\Delta_{X}\right)_{(3)}}\right)^{*} E}\right.}$ the curvature of $P$ and denote it by $R^{P}$.

Local trivializations of a bundle are induced by a parallel displacement along an admissible sequence satisfying an appropriate condition.

THEOREM 4.4. (i) Let $\pi: E \rightarrow X$ be a bundle and $P \in \mathscr{P} \mathscr{D}(\pi, \mathfrak{S})$. Suppose that $X$ is $\mathfrak{\Xi}$-connected and $\mathfrak{\Xi}_{(1)} \in \mathscr{S}_{X^{2}}\left(\Delta_{X}\right)$. Then for any $x \in X, \pi$ is a locally trivial bundle with standard fiber $E_{x}$.

(ii) Let $G$ be a topological group, $\pi: E \rightarrow X$ a principal G-bundle, and $P \in \mathscr{P} \mathscr{D}(\pi, \mathfrak{S})_{G}$. Suppose that $X$ is $\mathfrak{\subseteq}$-connected and $\mathfrak{S}_{(1)} \in \mathscr{S}_{X^{2}}\left(\Delta_{X}\right)$. Then $\pi$ is a locally trivial G-bundle. 
Proof. Firstly, we show (i). For $y \in X$, fix $c_{y} \in \mathfrak{S}_{y, x}$ and a neighborhood $V_{y}$ of $y$ in $X$ such that $V_{y} \times\{y\} \subset \Im_{(1)}$. A continuous map $\alpha_{y}: E\left\lceil_{V_{y}} \rightarrow V_{y} \times E_{x}\right.$ is defined by

$$
\alpha_{y}(v):=\left(\pi(v), P\left(c_{y}^{-} \bullet(y, \pi(v)), v\right)\right)
$$

for $v \in E\left\lceil_{V_{y}}\right.$. Then $\operatorname{pr}_{1} \circ \alpha_{y}=\pi\left\lceil_{V_{y}}\right.$. On the other hand, a continuous map $\kappa_{y}: V_{y} \times E_{x} \rightarrow E\left\lceil_{V_{y}}\right.$ is defined by

$$
\kappa_{y}(z, p):=P\left((z, y) \bullet c_{y}, p\right)
$$

for $(z, p) \in V_{y} \times E_{x}$. We can see that $\kappa_{y}=\left(\alpha_{y}\right)^{-1}$. Therefore, $\alpha_{y}$ is a local trivialization.

Secondly, we show (ii). Let $u \in E$. For $y \in X$, fix $c_{y} \in \Xi_{y, \pi(u)}$ and a neighborhood $V_{y}$ of $y$ in $X$ such that $V_{y} \times\{y\} \subset \Xi_{(1)}$. A continuous map $\alpha_{y}: E\left\lceil_{V_{y}} \rightarrow\right.$ $V_{y} \times G$ is defined by

$$
\alpha_{y}(v):=\left(\pi(v), T\left(u, P\left(c_{y}^{-} \bullet(y, \pi(v)), v\right)\right)\right)
$$

for $v \in E\left\lceil_{V_{y}}\right.$. Then $\operatorname{pr}_{1} \circ \alpha_{y}=\pi\left\lceil_{V_{y}}\right.$. For $(v, a) \in E\left\lceil_{V_{y}} \times G\right.$, we have

$$
\begin{aligned}
\alpha_{y}(v a) & =\left(\pi(v), T\left(u, P\left(c_{y}^{-} \bullet(y, \pi(v)), v a\right)\right)\right) \\
& =\left(\pi(v), T\left(u, P\left(c_{y}^{-} \bullet(y, \pi(v)), v\right)\right)\right) a=\alpha_{y}(u) a .
\end{aligned}
$$

On the other hand, a continuous map $\kappa_{y}: V_{y} \times G \rightarrow E\left\lceil_{V_{y}}\right.$ is defined by

$$
\kappa_{y}(z, a):=P\left((z, y) \bullet c_{y}, u a\right)
$$

for $(z, a) \in V_{y} \times G$. Then we can see that $\kappa_{y}=\left(\alpha_{y}\right)^{-1}$. Thus, $\alpha_{y}$ is a local trivialization.

Next we introduce morphisms preserving parallel displacements. Let $X$ and $X^{\prime}$ be topological spaces. For a continuous map $f: X \rightarrow X^{\prime}$, let $f^{\sqcup}: X^{\sqcup} \rightarrow X^{\prime}$ be a continuous map such that

$$
\left.f^{\sqcup}\right|_{X^{n+1}}=f^{n+1}:=f \times \cdots \times f: X^{n+1} \rightarrow X^{\prime n+1}
$$

for $n \geq 0$.

Definition 4.4. Let $\subseteq \in \mathscr{A} \mathscr{S}(X)$ and $\Xi^{\prime} \in \mathscr{A} \mathscr{S}\left(X^{\prime}\right)$. We say that $f: X \rightarrow X^{\prime}$ preserves $\mathfrak{\Xi}$ and $\mathbb{\Xi}^{\prime}$ if $f^{\sqcup}(\mathfrak{\Xi}) \subset \mathbb{S}^{\prime}$. Let $\pi: E \rightarrow X$ (resp. 
$\left.\pi^{\prime}: E^{\prime} \rightarrow X^{\prime}\right)$ be a bundle and $P \in \mathscr{P} \mathscr{D}(\pi, \mathfrak{S}) \quad\left(\right.$ resp. $\left.\quad P^{\prime} \in \mathscr{P} \mathscr{D}\left(\pi^{\prime}, \mathbb{S}^{\prime}\right)\right)$. Let $(h, f) \in \operatorname{Hom}\left(\pi, \pi^{\prime}\right)$ such that $f$ preserves $\subseteq$ and $\mathfrak{\Xi}^{\prime}$. We say that $(h, f)$ preserves $P$ and $P^{\prime}$ if

$$
h(P(\mathbf{x}, u))=P^{\prime}\left(f^{\sqcup}(\mathbf{x}), h(u)\right)
$$

for $(\mathbf{x}, u) \in\left(\left.p_{0}\right|_{\Im}\right)^{*} E$

We obtain a category whose objects are bundles with parallel displacement along admissible sequences and morphisms are bundle morphisms preserving parallel displacements.

Definition 4.5. Let $\pi$ (resp. $\pi^{\prime}$ ) be a $G$ (resp. $G^{\prime}$ )-bundle and $P \in$ $\mathscr{P} \mathscr{D}(\pi, \subseteq)_{G} \quad\left(\right.$ resp. $\left.P^{\prime} \in \mathscr{P} \mathscr{D}\left(\pi^{\prime}, \Xi^{\prime}\right)_{G^{\prime}}\right)$. For $(h, f, \rho) \in \operatorname{Hom}\left((\pi, G),\left(\pi^{\prime}, G^{\prime}\right)\right)$, we say that $(h, f, \rho)$ preserves $P$ and $P^{\prime}$ if $(h, f)$ preserves $P$ and $P^{\prime}$ as a bundle morphism.

Similarly, we obtain a category whose objects are $G$-bundles with $G$ compatible parallel displacement along admissible sequences and morphisms are homomorphisms preserving $G$-compatible parallel displacements, where $G$ runs throughout the whole topological groups and $\rho$ runs throughout the whole continuous group homomorphisms. For a map $f: X^{\prime} \rightarrow X$, a map $f^{*}: \mathscr{P}\left(X^{\sqcup}\right) \rightarrow \mathscr{P}\left(X^{\prime}\right)$ is defined by

$$
f^{*} A:=f^{*}(A):=\left(f^{\sqcup}\right)^{-1}(A)
$$

for $A \in \mathscr{P}\left(X^{\sqcup}\right)$. Let $\pi: E \rightarrow X$ be a bundle and $P \in \mathscr{P} \mathscr{D}(\pi, \subseteq)$. For $Y \subset X$, put $\Im\left\lceil_{Y}:=i_{Y, X}^{*}(\Im)\right.$ and

$$
P\left\lceil_{Y}:={ }_{E\left\lceil_{Y}\right.}|P|_{\left(\left.p_{0}\right|_{\Theta} \Gamma_{Y}\right)^{*} E\left\lceil_{Y}\right.} .\right.
$$

We call $P\left\lceil_{Y}\right.$ the restricted parallel displacement. For a continuous map $f: X^{\prime} \rightarrow X$, an induced parallel displacement $f^{*} P:\left(\left.p_{0}\right|_{f^{*} \Subset}\right)^{*} f^{*} E \rightarrow f^{*} E$ is defined by

$$
\begin{aligned}
\left(f^{*} P\right)\left(\left(x_{n}, \ldots, x_{0}\right),\left(x_{0}, u\right)\right): & =\left(x_{n}, P\left(f^{\sqcup}\left(x_{n}, \ldots, x_{0}\right), \bar{f}\left(x_{0}, u\right)\right)\right) \\
& =\left(x_{n}, P\left(f^{\sqcup}\left(x_{n}, \ldots, x_{0}\right), u\right)\right)
\end{aligned}
$$

for $\left(\left(x_{n}, \ldots, x_{0}\right),\left(x_{0}, u\right)\right) \in\left(\left.p_{0}\right|_{f *}\right)^{*} f^{*} E$. The following properties are fundamental and the proof is straightforward. 
Proposition 4.5. (i) The bundle map $\left(i_{\left.E\right|_{Y}, E}, i_{Y, X}\right)$ preserves $P\left\lceil_{Y}\right.$ and $P$.

(ii) The canonical bundle map $(\bar{f}, f)$ preserves $f^{*} P$ and $P$.

(iii) If $\pi$ is a G-bundle and $P$ is G-compatible, then $P\left\lceil_{Y}\right.$ and $f^{*} P$ are also G-compatible.

Let $\pi: E \rightarrow X$ be a principal $G$-bundle, $G^{\prime}$ a topological group, $\rho: G \rightarrow G^{\prime}$ a continuous group homomorphism, and $\pi^{\rho}$ the $G^{\prime}$-bundle associated with $\pi$. For $P \in \mathscr{P} \mathscr{D}(\pi, \subseteq)_{G}$ put

$$
P^{\rho}(\mathbf{x},[u, a]):=[P(\mathbf{x}, u), a]
$$

for $(\mathbf{x},[u, a]) \in\left(\left.p_{0}\right|_{\Subset}\right)^{*} E^{\rho}$.

Proposition 4.6. We have $P^{\rho} \in \mathscr{P} \mathscr{D}\left(\pi^{\rho}, \Im\right)_{G^{\prime}}$.

Proof. We can see

$$
\left(i d_{\Im} \times q_{G}^{E \times G^{\prime}}\right)^{-1}\left(\left(\left.p_{0}\right|_{\Subset}\right)^{*} E^{\rho}\right)=\left(\left(\left.p_{0}\right|_{\Xi}\right)^{*} E\right) \times G^{\prime} .
$$

Then, from Lemma 2.2, $q^{\prime}:={ }_{\left(\left.p_{0}\right|_{\odot}\right)^{*} E^{\rho}}\left|\left(i d_{\odot} \times q_{G}^{E \times G^{\prime}}\right)\right|_{\left(\left(\left.p_{0}\right|_{\Xi}\right)^{*} E\right) \times G^{\prime}}$ is an open map. Since $q^{\prime}$ is surjective, it is an identification. Thus, the continuity of $P^{\rho}$ follows from the equality $P^{\rho} \circ q^{\prime}=q_{G}^{E \times G^{\prime}} \circ\left(P \times i d_{G^{\prime}}\right)$. Since

$$
\begin{aligned}
P^{\rho}(\mathbf{x},[u, a] b) & =P^{\rho}(\mathbf{x},[u, a b])=[P(\mathbf{x}, u), a b] \\
& =[P(\mathbf{x}, u), a] b=P^{\rho}(\mathbf{x},[u, a]) b
\end{aligned}
$$

for $((\mathbf{x},[u, a]), b) \in\left(\left(p_{0} \mid \varsigma\right)^{*} E^{\rho}\right) \times G^{\prime}, P^{\rho}$ is $G^{\prime}$-compatible.

We call $P^{\rho}$ a $G^{\prime}$-compatible parallel displacement associated with $P$. By straightforward arguments, we obtain the following fundamental propositions, which will be needed in the proof of Theorem 8.1 (Classification Theorem).

Proposition 4.7. The homomorphism $\left(\theta^{\rho}, \rho\right) \in \operatorname{Hom}\left((\pi, G),\left(\pi^{\rho}, G^{\prime}\right)\right)$ preserves $P$ and $P^{\rho}$.

Proposition 4.8. Let $\pi: E \rightarrow X$ (resp. $\pi^{\prime}: E^{\prime} \rightarrow X$ ) be a principal $G$ (resp. $\left.G^{\prime}\right)$-bundle. Let $P \in \mathscr{P} \mathscr{D}(\pi, \mathfrak{S})_{G}$ and $P^{\prime} \in \mathscr{P} \mathscr{D}\left(\pi^{\prime}, \mathbb{S}\right)_{G^{\prime}}$. If $(h, \rho) \in \operatorname{Hom}((\pi, G)$, $\left.\left(\pi^{\prime}, G^{\prime}\right)\right)$ preserves $P$ and $P^{\prime}$, then $h^{\rho} \in \operatorname{Hom}_{X, G^{\prime}}\left(\left(\pi^{\rho}, G^{\prime}\right),\left(\pi^{\prime}, G^{\prime}\right)\right)$ preserves $P^{\rho}$ and $P^{\prime}$. 


\section{A Holonomy Group of the Parallel Displacement}

In this section, we define a holonomy group of the parallel displacement and study its fundamental properties. In particular, a relation between the holonomy group of a connection in the smooth category and the holonomy group of the parallel displacement induced by the smooth slicing function in Section 3 is studied. Moreover, we define a local holonomy group of the parallel displacement. In the smooth category, the Lie algebra of the holonomy group of a connection is spanned by the image of the curvature at every point of the holonomy bundle. As an analogue of this fact, we show that the local holonomy group is generated by the curvature of a parallel displacement. At the end of this section, we study a relation between the local holonomy group of a connection in the smooth category and the local holonomy group of the parallel displacement induced by the smooth slicing function in Section 3.

Let $\pi: E \rightarrow X$ be a principal $G$-bundle with translation function $T$ (see Section 2) and $P$ a $G$-compatible parallel displacement along $\subseteq$ in $\pi$, that is, $P \in \mathscr{P} \mathscr{D}(\pi, \mathfrak{S})_{G}$. For $x \in X$, we denote by $\mathfrak{S}_{x}$ the subset $\left(\left.\left.p_{\infty}\right|_{\mathfrak{S}} \hat{\times} p_{0}\right|_{\mathfrak{S}}\right)^{-1}(\{(x, x)\})$ of $\subseteq$ (see Definition 4.1).

Definition 5.1. For $u \in E$, a map $P^{u}: \Im_{\pi(u)} \rightarrow G$ is defined by

$$
P^{u}(\mathbf{x}):=T(u, P(\mathbf{x}, u))
$$

for $\mathbf{x} \in \mathfrak{S}_{\pi(u)}$. A subgroup

$$
\Phi^{u}:=\Phi^{u}(P):=P^{u}\left(\Im_{\pi(u)}\right)
$$

of $G$ is called the holonomy group of $P$ with reference point $u$. We assume that the topology of $\Phi^{u}$ is the relative topology induced from $G$.

Note that $\Xi_{\pi(u)}$ is a monoid with identity element $\pi(u)$ and $P^{u}$ is a monoid homomorphism. We show in Proposition 5.1 that $\Phi^{u}$ is in fact a subgroup of $G$. The following examples of holonomy groups are typical.

Example 5.1. Let $\operatorname{pr}_{1}: X \times G \rightarrow X, P^{\omega} \in \mathscr{P} \mathscr{D}_{G}\left(\mathrm{pr}_{1}, X^{\sqcup}\right)$ be as in Example 4.2 and $(x, a) \in X \times G$. For $\left(x, x_{n-1}, \ldots, x_{1}, x\right) \in \Xi_{x}$

$$
\begin{aligned}
\left(P^{\omega}\right)^{(x, a)}\left(x, x_{n-1}, \ldots, x_{1}, x\right) & =T\left((x, a), P^{\omega}\left(\left(x, x_{n-1}, \ldots, x_{1}, x\right),(x, a)\right)\right) \\
& =T((x, a),(x, a))=a^{-1} a=1_{G} .
\end{aligned}
$$

Thus, $\Phi^{(x, a)}\left(P^{\omega}\right)=\left\{1_{G}\right\}$. 
EXAMPLE 5.2 (cf. [14]). Let $\tilde{\pi}_{K}$ be the universal bundle which we have reviewed in Example 3.5, $\tilde{P}_{K}$ the universal parallel displacement as in Example 4.3, and $\left[v_{0}\right] \in \tilde{E}_{K}$. Since

$$
\begin{aligned}
\tilde{P}_{K}^{\left[v_{0}\right]}\left(v_{0}, x_{n-1}, \ldots, x_{1}, v_{0}\right) & =\tilde{P}_{K}\left(\left(v_{0}, x_{n-1}, \ldots, x_{1}, v_{0}\right),\left[v_{0}\right]\right) \\
& =\left[v_{0}, x_{n-1}, \ldots, x_{1}, v_{0}\right]
\end{aligned}
$$

for $\left[v_{0}, x_{n-1}, \ldots, x_{1}, v_{0}\right] \in \tilde{G}_{K}$, we have $\Phi^{\left[v_{0}\right]}\left(\tilde{P}_{K}\right)=\tilde{G}_{K}$.

Similar to the holonomy group of a connection in the smooth category, the following fundamental properties hold (for example, [12]).

Proposition 5.1. (i) For $u \in E, \Phi^{u}$ is a topological subgroup of $G$.

(ii) For $u, v \in E$ and $\mathbf{x} \in \mathfrak{S}$ such that $P(\mathbf{x}, u)=v$, we have $\Phi^{u}=\Phi^{v}$.

(iii) For $(u, a) \in E \times G, \Phi^{u a}=a^{-1} \Phi^{u} a$.

Proof. Firstly, we show (i). For $a=P^{u}(\mathbf{x}), b=P^{u}(\mathbf{y}) \in \Phi^{u}$, since

$$
\begin{aligned}
a b & =T(u, P(\mathbf{x}, u)) T(u, P(\mathbf{y}, u))=T(u, P(\mathbf{x}, u T(u, P(\mathbf{y}, u)))) \\
& =T(u, P(\mathbf{x}, P(\mathbf{y}, u)))=T(u, P(\mathbf{x} \bullet \mathbf{y}, u))
\end{aligned}
$$

and

$$
a P^{u}\left(\mathbf{x}^{-}\right)=T(u, P(\mathbf{x}, u)) T\left(u, P\left(\mathbf{x}^{-}, u\right)\right)=T\left(u, P_{\mathbf{x}}\left(P_{\mathbf{x}}^{-1}(u)\right)\right)=1_{G},
$$

we have $a b \in \Phi^{u}$ and $a^{-1}=P^{u}\left(\mathbf{x}^{-}\right) \in \Phi^{u}$. Thus, from the property of the relative topology, $\Phi^{u}$ is a topological subgroup of $G$.

Secondly, we show (ii). For $a=P^{u}(\mathbf{y}) \in \Phi^{u}$, since

$$
P\left(\mathbf{x} \bullet \mathbf{y} \bullet \mathbf{x}^{-}, v\right)=P\left(\mathbf{x}, P\left(\mathbf{y}, P\left(\mathbf{x}^{-}, v\right)\right)\right)=P(\mathbf{x}, P(\mathbf{y}, u))=P(\mathbf{x}, u a)=v a,
$$

we have $a=P^{v}\left(\mathbf{x} \bullet \mathbf{y} \bullet \mathbf{x}^{-}\right)$and $a \in \Phi^{v}$. Similarly, we have $\Phi^{v} \subset \Phi^{u}$.

Thirdly, we show (iii). For $b=T(u a, P(\mathbf{y}, u a)) \in \Phi^{u a}$, since

$$
b=T(u a, P(\mathbf{y}, u) a)=a^{-1} T(u, P(\mathbf{y}, u)) a=a^{-1} P^{u}(\mathbf{y}) a,
$$

we have $b \in a^{-1} \Phi^{u} a$, where $\mathbf{y} \in \Im_{\pi(u a)}=\Im_{\pi(u)}$. Similarly, we have $a^{-1} \Phi^{u} a \subset \Phi^{u a}$ and this completes the proof.

Let $\pi: E \rightarrow X$ be a principal $G$-bundle and $u \in E$. Let $I$ be a pseudodirected set and $\left(\Im_{i}\right)_{i \in I} \in \mathscr{A} \mathscr{S}(X)^{I}$ such that for any $i, j \in I$, if $i \leq j$, then $\Im_{j} \subset \Im_{i}$. For $i, j \in I$ with $i \leq j$, let $\eta_{j i}: \mathscr{P} \mathscr{D}\left(\pi, \Im_{i}\right)_{G} \rightarrow \mathscr{P} \mathscr{D}\left(\pi, \Im_{j}\right)_{G}\left(\operatorname{resp} . \zeta_{j i}: \operatorname{Hom}\left(\left(\Im_{i}\right)_{\pi(u)}, G\right)\right.$ 
$\left.\rightarrow \operatorname{Hom}\left(\left(\Xi_{j}\right)_{\pi(u)}, G\right)\right)$ be the restriction, that is, $\eta_{j i}(P)=\left.P\right|_{\left(\left.p_{0}\right|_{\mathfrak{I}_{j}}\right)^{*} E}$ (resp. $\left.\zeta_{j i}(f)=f \circ i_{\left.\left(\Im_{j}\right)_{\pi(u)},\left(\Im_{i}\right)_{\pi(u)}\right)}\right) \quad$ for $\quad P \in \mathscr{P} \mathscr{D}\left(\pi, \Im_{i}\right)_{G} \quad\left(\right.$ resp. $\left.\quad f \in \operatorname{Hom}\left(\left(\Im_{i}\right)_{\pi(u)}, G\right)\right)$, where $\operatorname{Hom}\left(\left(\mathfrak{\Xi}_{i}\right)_{\pi(u)}, G\right)$ is the set of monoid homomorphisms. Then $\left(\mathscr{P} \mathscr{D}\left(\pi, \Xi_{i}\right)_{G}, \eta_{j i}\right)_{(i, j) \in I^{2}}$ (resp. $\left.\left(\operatorname{Hom}\left(\left(\Xi_{i}\right)_{\pi(u)}, G\right), \zeta_{j i}\right)_{(i, j) \in I^{2}}\right)$ is an inductive system. For $i \in I$, let $\varphi_{i}: \mathscr{P} \mathscr{D}\left(\pi, \widetilde{\Xi}_{i}\right)_{G} \rightarrow \operatorname{Hom}\left(\left(\widetilde{\Xi}_{i}\right)_{\pi(u)}, G\right)$ be a map given by

$$
\varphi_{i}(P):=P^{u} .
$$

Since $\varphi_{j} \circ \eta_{j i}=\zeta_{j i} \circ \varphi_{i}$ for $i, j \in I$ with $i \leq j$, a map $\lim _{i} \varphi_{i}: \lim _{\longrightarrow} \mathscr{P} \mathscr{D}\left(\pi, \Im_{i}\right)_{G}$ $\rightarrow \lim _{\longrightarrow} \operatorname{Hom}\left(\left(\Xi_{i}\right)_{\pi(u)}, G\right)$ can be defined. A map $\Xi_{I}: \lim _{\longrightarrow} \operatorname{Hom}\left(\left(\Xi_{i}\right)_{\pi(u)}, G\right) \rightarrow$ $\left\{G^{\prime} \subset G \mid G^{\prime}\right.$ : submonoid of $\left.G\right\}$ is defined by

$$
\Xi_{I}\left([f]_{I}\right):=\bigcap_{f^{\prime} \in[f]_{I}} \operatorname{Im} f^{\prime} .
$$

Put $\Phi_{I}^{u}:=\Xi_{I} \circ \lim _{\longrightarrow} \varphi_{i}$. Then $\Phi_{I}^{u}\left([P]_{I}\right)$ is a subgroup of $G$ for $[P]_{I} \in$ $\lim _{\longrightarrow} \mathscr{P} \mathscr{D}\left(\pi, \Im_{i}\right)_{G}$. We call $\Phi_{I}^{u}\left([P]_{I}\right)$ the holonomy group of $[P]_{I}$. For $i \in I$, let $\overrightarrow{\psi_{i}}: S F_{\text {inv }}\left(\pi, U_{i}\right)_{G} \rightarrow \mathscr{P} \mathscr{D}\left(\pi, \subseteq_{i}\right)_{G}$ be a map given by $\psi_{i}(\omega):=P^{\omega}$. Since $\psi_{j} \circ \rho_{j i}=$ $\eta_{j i} \circ \psi_{i}$ for $i, j \in I$ with $i \leq j$, a map $\lim _{i} \psi_{i}: \lim _{\longrightarrow} S F_{\text {inv }}\left(\pi, U_{i}\right)_{G} \rightarrow \lim _{\longrightarrow} \mathscr{P} \mathscr{D}\left(\pi, \Xi_{i}\right)_{G}$ can be defined. In particular, we consider $\Phi_{\mathscr{N}_{X^{2}}\left(\Delta_{X}\right)}^{u}\left(\left[P^{\omega}\right]_{\mathscr{N}_{X^{2}}\left(\Delta_{X}\right)}\right)$ as a holonomy group of a topological connection $[\omega]_{\mathcal{N}_{X^{2}}\left(\Delta_{X}\right)} \in \lim _{\longrightarrow} S F_{\text {inv }}(\pi, U)_{G}$ in the sense of Asada, where $\mathscr{N}_{X^{2}}\left(\Delta_{X}\right)$ is the set of neighborhoods of $\Delta_{X}$ in $X^{2}$.

Definition 5.2. Let $\pi: E \rightarrow X$ be a smooth principal $G$-bundle, $X$ a Riemannian manifold, and $H$ a connection in $E$ (see Example 3.7). For any piecewise smooth curve $c:[0,1] \rightarrow X$, let $\tau_{c}^{H}: E_{c(0)} \rightarrow E_{c(1)}$ be the parallel displacement along $c$ derived from $H$. For $u \in E$, put

$$
\begin{aligned}
\Psi_{\mathrm{geo}}^{u}(H):= & \left\{T\left(u, \tau_{c}^{H}(u)\right) \in G \mid c\right. \\
& \text { is a closed curve consisting of geodesic segments }\} .
\end{aligned}
$$

The following proposition shows a relation between the holonomy group of a connection and the holonomy group of the parallel displacement induced by the smooth slicing function in Example 3.7.

Proposition 5.2. Let $\pi: E \rightarrow X$ be a smooth principal G-bundle, $X$ a Riemannian manifold, $H$ a connection in $E$, and $\omega^{H} \in S F_{\mathrm{inv}}\left(\pi, U_{\mathscr{r}}\right)_{G}$ as in Example 3.7, where $\mathscr{V}$ is an open covering of $X$ which consists of strongly convex sets and $U_{\mathscr{r}}=\bigcup_{V \in \mathscr{V}} V \times V$. Then $\Psi_{\mathrm{geo}}^{u}(H)=\Phi^{u}\left(P^{\omega^{H}}\right)$, and consequently $\Psi_{\mathrm{geo}}^{u}(H)=$ $\Phi_{\mathscr{S} \mathscr{C}(X)}^{u}\left(\left[P^{\omega^{H}}\right]_{\mathscr{S} \mathscr{C}(X)}\right)$, where $\mathscr{S} \mathscr{C}(X)$ denotes the set of open coverings of $X$ which consist of strongly convex sets, preordered by $\mathscr{V} \leq \mathscr{W}$ if $\mathscr{W}$ is a refinement of $\mathscr{V}$. 
Proof. Let $\left(P^{\omega^{H}}\right)^{u}\left(x_{n}, \ldots, x_{1}, x_{0}\right) \in \Phi^{u}\left(P^{\omega^{H}}\right)$, where $x_{n}=x_{0}=\pi(u)$. Then there exists a unique family of shortest geodesics $\left(\gamma_{i}:[0,1] \rightarrow X\right)_{i \in\{0, \ldots, n-1\}}$ such that $\gamma_{i}(0)=x_{i}$ and $\gamma_{i}(1)=x_{i+1}$. Since $\tau_{\gamma_{i}}^{H}=\omega_{x_{i+1} x_{i}}^{H}$ for $i \in\{0, \ldots, n-1\}$, we have

$$
\begin{aligned}
\left(P^{\omega^{H}}\right)^{u}\left(x_{n}, \ldots, x_{1}, x_{0}\right) & =T\left(u,\left(\omega_{x_{n} x_{n-1}}^{H} \circ \cdots \circ \omega_{x_{1} x_{0}}^{H}\right)(u)\right) \\
& =T\left(u,\left(\tau_{\gamma_{n-1}}^{H} \circ \cdots \circ \tau_{\gamma_{0}}^{H}\right)(u)\right)=T\left(u, \tau_{\gamma_{n-1} \cdots \gamma_{1} \gamma_{0}}^{H}(u)\right) \in \Psi_{\text {geo }}^{u}(H) .
\end{aligned}
$$

Thus $\Phi^{u}\left(P^{\omega^{H}}\right) \subset \Psi_{\text {geo }}^{u}(H)$.

Conversely, let $T\left(u, \tau_{\gamma_{n} \cdots \gamma_{1}}^{H}(u)\right) \in \Psi_{\text {geo }}^{u}(H)$, where $\left(\gamma_{i}:[0,1] \rightarrow X\right)_{i \in\{1, \ldots, n\}}$ is a family of geodesics. For $i \in\{1, \ldots, n\}, \quad\left(\gamma_{i}^{-1}(V)\right)_{V \in \mathscr{V}}$ is an open covering of $[0,1]$. Let $\delta^{i}>0$ be a Lebesgue's number of $\left(\gamma_{i}^{-1}(V)\right)_{V \in \mathscr{V}}$ and $0=t_{0}^{i}<$ $t_{1}^{i}<\cdots<t_{k_{i}}^{i}=1$ a partition of $[0,1]$ such that $t_{j}^{i}-t_{j-1}^{i}<\delta^{i}$. Then there exists a sequence $V_{1}^{i}, \ldots, V_{k_{i}}^{i} \in \mathscr{V}$ such that $\gamma_{i}\left(\left[t_{j-1}^{i}, t_{j}^{i}\right]\right) \subset V_{j}$ for $j \in\left\{1, \ldots, k_{i}\right\}$. Therefore, $\left(\gamma_{i}(1), \gamma_{i}\left(t_{k_{i}-1}^{i}\right), \ldots, \gamma_{i}\left(t_{1}^{i}\right), \gamma_{i}(0)\right) \in\left\langle U_{\mathscr{V}}\right\rangle$. Since

we have



$$
\begin{aligned}
& \tau_{\gamma_{n} \cdots \gamma_{1}}^{H}=\tau_{\gamma_{n}}^{H} \circ \cdots \circ \tau_{\gamma_{1}}^{H}
\end{aligned}
$$

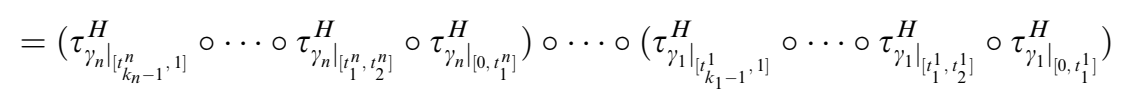

$$
\begin{aligned}
& =\left(\omega_{\gamma_{n}(1), \gamma_{n}\left(t_{k_{n}-1}^{n}\right)}^{H} \circ \cdots \circ \omega_{\gamma_{n}\left(t_{2}^{n}\right), \gamma_{n}\left(t_{1}^{n}\right)}^{H} \circ \omega_{\gamma_{n}\left(t_{1}^{n}\right), \gamma_{n}(0)}^{H}\right) \circ \cdots \\
& \circ\left(\omega_{\gamma_{1}(1), \gamma_{1}\left(t_{k_{1}-1}^{1}\right)}^{H} \circ \cdots \circ \omega_{\gamma_{1}\left(t_{2}^{1}\right), \gamma_{1}\left(t_{1}^{1}\right)}^{H} \circ \omega_{\gamma_{1}\left(t_{1}^{1}\right), \gamma_{1}(0)}^{H}\right) \\
& =P_{\left(\gamma_{n}(1), \gamma_{n}\left(t_{k_{n}-1}^{n}\right), \ldots, \gamma_{1}\left(t_{1}^{1}\right), \gamma_{1}(0)\right)}^{\omega^{H}} \text {. }
\end{aligned}
$$

Thus,

$$
T\left(u, \tau_{\gamma_{n} \cdots \gamma_{1}}^{H}(u)\right)=\left(P^{\omega^{H}}\right)^{u}\left(\gamma_{n}(1), \gamma_{n}\left(t_{k_{n}-1}^{n}\right), \ldots, \gamma_{1}\left(t_{1}^{1}\right), \gamma_{1}(0)\right) \in \Phi^{u}\left(P^{\omega^{H}}\right)
$$

and $\Psi_{\mathrm{geo}}^{u}(H) \subset \Phi^{u}\left(P^{\omega^{H}}\right)$.

Next we study local holonomy groups for the parallel displacements. For a symmetric subspace $U \subset X^{2}$ with $\Delta_{X} \subset U$ and $x \in X$, put

$$
\langle U\rangle_{x}^{1}:=\left\{\left(x, x_{n-1}, \ldots, x_{1}, x\right) \in\langle U\rangle_{x} \mid \forall k \in\{1, \ldots, n-1\}:\left(x_{k}, x\right) \in U\right\} .
$$

Then $\langle U\rangle_{x}^{1}$ is a submonoid of $\langle U\rangle_{x}$. Let $\pi: E \rightarrow X$ be a principal $G$-bundle and $P \in \mathscr{P} \mathscr{D}(\pi, \Xi)$. 
Definition 5.3. For $u \in E$, we call a subgroup

$$
\Phi^{u, 1}(P):=P^{u}\left(\left\langle\Xi_{(1)}\right\rangle_{\pi(u)}^{1}\right)
$$

of $\Phi^{u}(P)$ the local holonomy group of $P$ with reference point $u$.

Let $\operatorname{Hom}_{\text {inv }}\left(\langle U\rangle_{x}^{1}, G\right)$ be the set of $f \in \operatorname{Hom}\left(\langle U\rangle_{x}^{1}, G\right)$ such that for $\left(x_{n}, x_{n-1}, \ldots, x_{1}, x_{0}\right) \in\langle U\rangle_{x}^{1}$,

$$
f\left(x_{n}, x_{n-1}, \ldots, x_{i}, \ldots, x_{1}, x_{0}\right)=f\left(x_{n}, x_{n-1}, \ldots, \hat{x}_{i}, \ldots, x_{1}, x_{0}\right)
$$

if $x_{i+1}=x_{i-1}$ or $x_{i}=x_{i-1}$ for some $i \in\{1, \ldots, n\}$, where the symbol $\hat{x}$ denotes deletion of $x$. Note that $\operatorname{Im} f$ is a subgroup of $G$ for $f \in \operatorname{Hom}_{\text {inv }}\left(\langle U\rangle_{x}^{1}, G\right)$. A map

$$
\Xi^{x, 1}: \operatorname{Hom}_{\text {inv }}\left(\langle U\rangle_{x}^{1}, G\right) \rightarrow\left\{G^{\prime} \subset G \mid G^{\prime}: \text { subgroup of } G\right\}
$$

is given by

$$
\Xi^{x, 1}(f):=\operatorname{Im} f
$$

for $f \in \operatorname{Hom}_{\text {inv }}\left(\langle U\rangle_{x}^{1}, G\right)$. A map $\varsigma^{u}: \mathscr{P} \mathscr{D}(\pi, \subseteq)_{G} \rightarrow \operatorname{Hom}_{\text {inv }}\left(\left\langle\Im_{(1)}\right\rangle_{\pi(u)}^{1}, G\right)$ is given by

$$
\varsigma^{u}(P):=P^{u} \circ i_{\left\langle\mathfrak{\subseteq}_{(1)}\right\rangle_{\pi(u)}^{1}, \mathfrak{\subseteq}_{\pi(u)}} .
$$

Then we can consider $\Phi^{u, 1}$ as a map $\mathscr{P} \mathscr{D}(\pi, \subseteq)_{G} \rightarrow\left\{G^{\prime} \subset G \mid G^{\prime}\right.$ : subgroup of $\left.G\right\}$ such that $\Phi^{u, 1}=\Xi^{\pi(u), 1} \circ \varsigma^{u}$.

Let $\mathscr{Q}^{x}(U, G)$ be the set of continuous maps $f:\left(\langle U\rangle_{x}\right)_{(3)} \rightarrow G$ such that

(1) for $\left(x_{3}, x_{2}, x_{1}, x_{0}\right) \in\left(\langle U\rangle_{x}\right)_{(3)}, f\left(x_{3}, x_{2}, x_{1}, x_{0}\right)=1_{G}$ if $x_{i}=x_{i-1}$ for some $i \in\{1,2,3\}$,

(2) $f\left(\mathbf{x}^{-}\right)=f(\mathbf{x})^{-1}$ for $\mathbf{x} \in\left(\langle U\rangle_{x}\right)_{(3)}$.

Let $\langle\langle S\rangle\rangle$ denote the subgroup of $G$ generated by a subset $S \subset G$. A map

$$
\Xi^{x, \text { gen }}: \mathscr{Q}^{x}(U, G) \rightarrow\left\{G^{\prime} \subset G \mid G^{\prime}: \text { subgroup of } G\right\}
$$

is given by $\Xi^{x, \operatorname{gen}}(f):=\langle\langle\operatorname{Im} f\rangle\rangle$. We show that there exists a one-toone correspondence between $\operatorname{Hom}_{\text {inv }}\left(\langle U\rangle_{x}^{1}, G\right)$ and $\mathscr{Q}^{x}(U, G)$. Two maps $\vartheta: \operatorname{Hom}_{\text {inv }}\left(\langle U\rangle_{x}^{1}, G\right) \rightarrow \mathscr{Q}^{x}(U, G)$ and $\sigma: \mathscr{Q}^{x}(U, G) \rightarrow \operatorname{Hom}_{\text {inv }}\left(\langle U\rangle_{x}^{1}, G\right)$ are given by

$$
\vartheta(f):=f \circ i_{\left(\langle U\rangle_{x}\right)_{(3)},\langle U\rangle_{x}^{1}}
$$


for $f \in \operatorname{Hom}_{\mathrm{inv}}\left(\langle U\rangle_{x}^{1}, G\right)$ and

$$
\begin{aligned}
\sigma\left(f^{\prime}\right) & \left(x_{n}, x_{n-1}, \ldots, x_{1}, x_{0}\right) \\
& := \begin{cases}1_{G} & \text { if } n \in\{0,1,2\} \\
f^{\prime}\left(x, x_{n-1}, x_{n-2}, x\right) \cdots f^{\prime}\left(x, x_{3}, x_{2}, x\right) f^{\prime}\left(x, x_{2}, x_{1}, x\right) & \text { if } n \geq 3\end{cases}
\end{aligned}
$$

for $f^{\prime} \in \mathscr{Q}^{x}(U, G)$ and $\left(x_{n}, x_{n-1}, \ldots, x_{1}, x_{0}\right) \in\langle U\rangle_{x}^{1}$. For $f^{\prime} \in \mathscr{Q}^{x}(U, G)$ we have $\vartheta\left(\sigma\left(f^{\prime}\right)\right)=f^{\prime}$. Conversely, for $f \in \operatorname{Hom}_{\text {inv }}\left(\langle U\rangle_{x}^{1}, G\right)$ and $\left(x_{n}, x_{n-1}, \ldots, x_{1}, x_{0}\right) \in$ $\langle U\rangle_{x}^{1}$, we get

$$
\sigma(\vartheta(f))\left(x_{n}, x_{n-1}, \ldots, x_{1}, x_{0}\right)=1_{G}=f\left(x_{n}, x_{n-1}, \ldots, x_{1}, x_{0}\right)
$$

if $n \in\{0,1,2\}$, and

$$
\begin{aligned}
\sigma(\vartheta(f))\left(x_{n}, x_{n-1}, \ldots, x_{1}, x_{0}\right) \\
\quad=\vartheta(f)\left(x, x_{n-1}, x_{n-2}, x\right) \cdots \vartheta(f)\left(x, x_{3}, x_{2}, x\right) \vartheta(f)\left(x, x_{2}, x_{1}, x\right) \\
\quad=f\left(x, x_{n-1}, x_{n-2}, x\right) \cdots f\left(x, x_{3}, x_{2}, x\right) f\left(x, x_{2}, x_{1}, x\right) \\
\quad=f\left(\left(x, x_{n-1}, x_{n-2}, x\right) \bullet \cdots \bullet\left(x, x_{3}, x_{2}, x\right) \bullet\left(x, x_{2}, x_{1}, x\right)\right) \\
\quad=f\left(x, x_{n-1}, x_{n-2}, x, \ldots, x, x_{3}, x_{2}, x, x_{2}, x_{1}, x\right) \\
\quad=f\left(x, x_{n-1}, x_{n-2}, \ldots, x_{3}, x_{2}, x_{1}, x\right)
\end{aligned}
$$

if $n \geq 3$. Thus $\sigma=\vartheta^{-1}$. Note that $\Xi^{x, 1}=\Xi^{x, \text { gen }} \circ \vartheta$.

For $\mathfrak{S} \in \mathscr{A} \mathscr{S}(X)$, we denote by $\mathscr{R}(\pi, \mathfrak{S})$ the set of continuous maps $h:\left(\left.p_{0}\right|_{\left(\Im_{\Delta_{X}}\right)_{(3)}}\right)^{*} E \rightarrow E$ such that

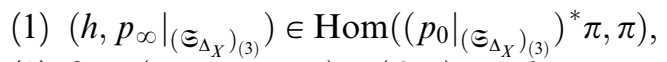

(2) for $\left(x_{3}, x_{2}, x_{1}, x_{0}\right) \in\left(\mathfrak{S}_{x_{0}}\right)_{(3)}, \quad h_{\left(x_{3}, x_{2}, x_{1}, x_{0}\right)}=i d_{E_{x_{0}}}$ if $x_{i}=x_{i-1}$ for some $i \in\{1,2,3\}$,

(3) $h_{\mathbf{x}^{-}}=h_{\mathbf{x}}^{-1}$ for $\mathbf{x} \in\left(\Xi_{x}\right)_{(3)}$,

where $h_{\mathbf{x}}:=h(\mathbf{x}, \cdot): E_{x} \rightarrow E_{x}$ for $\mathbf{x} \in\left(\Im_{x}\right)_{(3)}$. For a $G$-bundle $\pi$, we denote by $\mathscr{R}(\pi, \mathfrak{\Xi})_{G}$ the set of $h \in \mathscr{R}(\pi, \mathfrak{\Xi})$ such that $\left(h,\left.p_{\infty}\right|_{\left.\left(\subseteq_{\Delta_{X}}\right)_{(3)}\right)}\right)$ is a $G$-morphism. A map $\varrho: \mathscr{P} \mathscr{D}(\pi, \subseteq) \rightarrow \mathscr{R}(\pi, \subseteq)$ is given by assigning to $P \in \mathscr{P} \mathscr{D}(\pi, \subseteq)$ the restriction

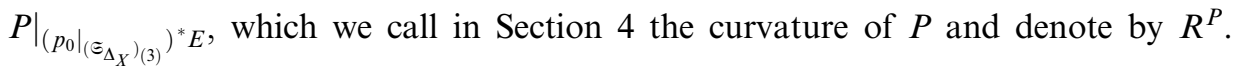
Note that $\left.\varrho(\mathscr{P} \mathscr{D}(\pi, \subseteq))_{G}\right) \subset \mathscr{R}(\pi, \subseteq)_{G}$. Let $\pi$ be a principal $G$-bundle, $u \in E$, and $h \in \mathscr{R}(\pi, \Im)_{G}$. Then a continuous map $h^{u}:\left(\Im_{\pi(u)}\right)_{(3)} \rightarrow G$ is given by

$$
h^{u}(\mathbf{x}):=T(u, h(\mathbf{x}, u))
$$


for $\mathbf{x} \in\left(\mathfrak{S}_{\pi(u)}\right)_{(3)}$. A map $\varepsilon^{u}: \mathscr{R}\left(\pi, \mathfrak{S}_{G} \rightarrow \mathscr{2}^{\pi(u)}\left(\mathfrak{S}_{(1)}, G\right)\right.$ is given by

$$
\varepsilon^{u}(h):=h^{u} \circ i_{\left(\left\langle\mathfrak{\subseteq}_{(1)}\right\rangle_{\pi(u)}\right)_{(3)},\left(\mathfrak{\Xi}_{\pi(u)}\right)_{(3)}} .
$$

We have $\vartheta \circ \varsigma^{u}=\varepsilon^{u} \circ \varrho$. Put $\Omega^{u}:=\Xi^{\pi(u), \text { gen }} \circ \varepsilon^{u}$. In the smooth category, the Lie algebra of the holonomy group of a connection is spanned by the image of the curvature at every point of the holonomy bundle. We obtain an analogue of this fact.

Proposition 5.3. For $P \in \mathscr{P} \mathscr{D}(\pi, \subseteq)_{G}, \Phi^{u, 1}(P)=\Omega^{u}\left(R^{P}\right)$.

Proof. Since

$$
\Xi^{\pi(u), 1} \circ \varsigma^{u}=\Xi^{\pi(u), \text { gen }} \circ \varepsilon^{u} \circ \varrho=\Xi^{\pi(u), \text { gen }} \circ \vartheta \circ \varsigma^{u}=\Xi^{\pi(u), \text { gen }} \circ \varepsilon^{u} \circ \varrho,
$$

we have the conclusion.

Let $I$ be a pseudodirected set and $\left(\Im_{i}\right)_{i \in I} \in \mathscr{A} \mathscr{S}(X)^{I}$ such that for any $i, j \in I$, if $i \leq j$, then $\Xi_{j} \subset \Im_{i}$. For $i, j \in I$ with $i \leq j$ and $x \in X$, let $\xi_{j i}: \operatorname{Hom}_{\mathrm{inv}}\left(\left\langle\left(\Xi_{i}\right)_{(1)}\right\rangle_{x}^{1}, G\right) \rightarrow \operatorname{Hom}_{\mathrm{inv}}\left(\left\langle\left(\Xi_{j}\right)_{(1)}\right\rangle_{x}^{1}, G\right)$ be the restriction. A map

$$
\Xi_{I}^{x, 1}: \underset{i}{\lim _{\vec{i}}} \operatorname{Hom}_{\mathrm{inv}}\left(\left\langle\left(\Xi_{i}\right)_{(1)}\right\rangle_{x}^{1}, G\right) \rightarrow\left\{G^{\prime} \subset G \mid G^{\prime}: \text { subgroup of } G\right\}
$$

is defined by

$$
\Xi_{I}^{x, 1}\left([f]_{I}\right):=\bigcap_{f^{\prime} \in[f]_{I}} \operatorname{Im} f^{\prime}
$$

for $[f]_{I} \in \lim _{\longrightarrow} \operatorname{Hom}_{\mathrm{inv}}\left(\left\langle\left(\mathfrak{\Im}_{i}\right)_{(1)}\right\rangle_{x}^{1}, G\right)$.

For $i, \overrightarrow{j \in I}$ with $i \leq j$ and $x \in X$, let $\kappa_{j i}: \mathscr{Q}^{x}\left(\left(\Im_{i}\right)_{(1)}, G\right) \rightarrow \mathscr{Q}^{x}\left(\left(\Im_{j}\right)_{(1)}, G\right)$ be the restriction. A map

$$
\Xi_{I}^{x, \text { gen }}: \lim _{i} \mathscr{Q}^{x}\left(\left(\Im_{i}\right)_{(1)}, G\right) \rightarrow\left\{G^{\prime} \subset G \mid G^{\prime}: \text { subgroup of } G\right\}
$$

is defined by

$$
\Xi_{I}^{x, \text { gen }}\left([f]_{I}\right):=\bigcap_{f^{\prime} \in[f]_{I}}\left\langle\left\langle\operatorname{Im} f^{\prime}\right\rangle\right\rangle
$$

For the maps $\vartheta_{i}: \operatorname{Hom}_{\text {inv }}\left(\left\langle\left(\Xi_{i}\right)_{(1)}\right\rangle_{x}^{1}, G\right) \rightarrow \mathscr{Q}^{x}\left(\left(\Im_{i}\right)_{(1)}, G\right)$ and $\sigma_{i}: \mathscr{V}^{x}\left(\left(\Theta_{i}\right)_{(1)}, G\right)$ $\rightarrow \operatorname{Hom}_{\mathrm{inv}}\left(\left\langle\left(\Theta_{i}\right)_{(1)}\right\rangle_{x}^{1}, G\right)$, since $\sigma_{i}=\vartheta_{i}^{-1}$ for $i \in I, \lim _{i} \sigma_{i}=\left(\lim _{i} \vartheta_{i}\right)^{-1}$. 
Proposition 5.4. For $[f]_{I} \in \lim _{\longrightarrow} \operatorname{Hom}_{\text {inv }}\left(\left\langle\left(\Im_{i}\right)_{(1)}\right\rangle_{x}^{1}, G\right), \quad \Xi_{I}^{x, 1}\left([f]_{I}\right)=$ $\Xi_{I}^{x, \operatorname{gen}}\left(\left(\lim _{\underline{i}} \vartheta_{i}\right)\left([f]_{I}\right)\right)$.

Proof. Note that $\operatorname{Im} f=\langle\langle\operatorname{Im} \vartheta(f)\rangle\rangle$. Let $a \in \Xi_{I}^{x, 1}\left([f]_{I}\right)$. Then, for any $f^{\prime} \in[f]_{I}, \quad a \in \operatorname{Im} f^{\prime}$. For $f^{\prime \prime} \in\left(\lim _{\longrightarrow} \vartheta_{i}\right)\left([f]_{I}\right)$ with $f^{\prime \prime} \in \mathscr{Q}^{x}\left(\left(\Im_{i}\right)_{(1)}, G\right)$, since $\sigma_{i}\left(f^{\prime \prime}\right) \in[f]_{I}, a \in\left\langle\left\langle\operatorname{Im} \vartheta_{i}\left(\sigma_{i}\left(f^{\prime \prime}\right)\right)\right\rangle\right\rangle=\left\langle\left\langle\operatorname{Im} f^{\prime \prime}\right\rangle\right\rangle$. Thus $a \in \Xi_{I}^{x, \operatorname{gen}}\left(\left(\lim _{i} \vartheta_{i}\right)\left([f]_{I}\right)\right)$ and $\Xi_{I}^{x, 1}\left([f]_{I}\right) \subset \Xi_{I}^{x, \text { gen }}\left(\left(\lim _{\longrightarrow} \vartheta_{i}\right)\left([f]_{I}\right)\right)$. By a similar argument, we have $\Xi_{I}^{x, 1}\left([f]_{I}\right) \supset \Xi_{I}^{x, \text { gen }}\left(\left(\lim _{\longrightarrow} \vartheta_{i}\right)\left([f]_{I}\right)\right)$.

For the maps $\varsigma_{i}^{u}: \mathscr{P} \mathscr{D}\left(\pi, \Im_{i}\right)_{G} \rightarrow \operatorname{Hom}_{\text {inv }}\left(\left\langle\left(\Im_{i}\right)_{(1)}\right\rangle_{\pi(u)}^{1}, G\right)$, since $\varsigma_{j}^{u} \circ \eta_{j i}$ $=\xi_{j i} \circ \varsigma_{i} \quad$ for $\quad i, j \in I$ with $i \leq j, \quad$ a $\operatorname{map} \lim _{\longrightarrow} \varsigma_{i}^{u}: \lim _{\longrightarrow} \mathscr{P} \mathscr{D}\left(\pi, \Im_{i}\right)_{G} \rightarrow$ $\lim _{\longrightarrow} \operatorname{Hom}_{\text {inv }}\left(\left\langle\left(\Theta_{i}\right)_{(1)}\right\rangle_{\pi(u)}^{1}, G\right)$ can be defined. Put $\Phi_{I}^{u, \vec{u}: i}:=\Xi_{I}^{\pi(\overrightarrow{u, 1}} i \lim _{\longrightarrow} \varsigma_{i}^{u}$. Then $\overrightarrow{\Phi_{I}^{u}, 1}\left([P]_{I}\right)$ is a subgroup of $\Phi_{I}^{u}\left([P]_{I}\right)$ for $[P]_{I} \in \lim _{\longrightarrow} \mathscr{P} \mathscr{D}\left(\pi, \Im_{i}\right)_{G}$. We call $\Phi_{I}^{u, 1}\left([P]_{I}\right)$ the local holonomy group of $[P]_{I}$ with reference point $u$.

For $i, j \in I$ with $i \leq j$, let $\zeta_{j i}: \mathscr{R}\left(\pi, \Im_{i}\right)_{G} \rightarrow \mathscr{R}\left(\pi, \Xi_{j}\right)_{G}$ be the restriction. For the maps $\varrho_{i}: \mathscr{P} \mathscr{D}\left(\pi, \Im_{i}\right) \rightarrow \mathscr{R}\left(\pi, \Im_{i}\right)$, since $\varrho_{j} \circ \eta_{j i}=\zeta_{j i} \circ \varrho_{i}$ for $i, j \in I$ with $i \leq j$, a map $\lim _{i} \varrho_{i}: \lim _{\rightarrow} \mathscr{P} \mathscr{D}\left(\pi, \Xi_{i}\right) \rightarrow \lim _{\rightarrow} \mathscr{R}\left(\pi, \Xi_{i}\right)$ can be defined. We call $\left(\lim _{i} \varrho_{i}\right)\left([P]_{I}\right)=\left[R^{P}\right]_{I}$ the curvature of $[P]_{I} \in \lim _{\longrightarrow} \mathscr{P} \mathscr{D}\left(\pi, \Im_{i}\right)$. Let $\pi$ be a $G$-bundle. We can also define a map $\lim _{\longrightarrow} \mathscr{P} \mathscr{D}\left(\pi, \Im_{i}\right)_{G} \rightarrow \lim _{\longrightarrow} \mathscr{R}\left(\pi, \Im_{i}\right)_{G}$.

For the maps $\varepsilon_{i}^{u}: \mathscr{R}\left(\pi, \vec{\Xi}_{i}\right)_{G} \rightarrow \mathscr{2}^{\pi(u)}\left(\left(\widetilde{\Xi}_{i}\right)_{(1)}, G\right)$, since $\varepsilon_{j}^{u} \circ \zeta_{j i}=\kappa_{j i} \circ \varepsilon_{i}^{u}$ for $i, j \in I$ with $i \leq j$, a map $\lim _{\longrightarrow} \varepsilon_{i}^{u}: \lim _{\longrightarrow} \mathscr{R}\left(\pi, \Xi_{i}\right)_{G} \rightarrow \lim _{\longrightarrow} \mathscr{2}^{\pi(u)}\left(\left(\Im_{i}\right)_{(1)}, G\right)$ can be defined. Put $\Omega_{I}^{u}:=\Xi_{I}^{\pi(u), \text { gen }} \underset{\circ}{\longrightarrow} \lim _{i} \varepsilon_{i}^{u}$.

Proposition 5.5. For $[P]_{I} \in \lim _{i} \mathscr{P} \mathscr{D}\left(\pi, \Im_{i}\right), \Phi_{I}^{u, 1}\left([P]_{I}\right)=\Omega_{I}^{u}\left(\left[R^{P}\right]_{I}\right)$.

Proof. Since $\vartheta_{i} \circ \varsigma_{i}^{u}=\varepsilon_{i}^{u} \circ \varrho_{i}$ for any $i \in I, \lim _{\longrightarrow} \vartheta_{i} \circ \lim _{\longrightarrow} \varsigma_{i}^{u}=\lim _{i} \varepsilon_{i}^{u} \circ \lim _{i} \varrho_{i}$. Then, from Proposition 5.4, we get

$$
\begin{aligned}
\Xi_{I}^{\pi(u), 1}\left(\left(\lim _{\vec{i}} \varsigma_{i}^{u}\right)\left([P]_{I}\right)\right) & =\Xi_{I}^{\pi(u), \operatorname{gen}}\left(\left(\lim _{\vec{i}} \vartheta_{i}\right)\left(\left(\lim _{\vec{i}} \varsigma_{i}^{u}\right)\left([P]_{I}\right)\right)\right) \\
& =\Xi_{I}^{\pi(u), \operatorname{gen}}\left(\left(\lim _{\vec{i}} \varepsilon_{i}^{u}\right)\left(\left[R^{P}\right]_{I}\right)\right)
\end{aligned}
$$

and obtain the conclusion.

For $U \subset X^{2}$ such that $\Delta_{X} \subset U$, put

$$
V\left(E^{2}\left\lceil_{U}\right):=\left\{(w, v, u) \in E^{3} \mid(v, u),(w, u),(w, v) \in E^{2}\left\lceil_{U}\right\}\right.\right.
$$


We denote by $C^{2}(\pi, U)_{G}$ the set of continuous maps $f: V\left(E^{2}\left\lceil_{U}\right) \rightarrow G\right.$ such that

(1) $f(w, v, u)=1_{G}$ if $w=v$ or $v=u$ for $(w, v, u) \in V\left(E^{2}\left\lceil_{U}\right)\right.$,

(2) $f(w a, v b, u c)=b^{-1} f(w, v, u) b$ for $(w, v, u) \in V\left(E^{2}\left\lceil_{U}\right)\right.$ and $a, b, c \in G$.

A map $\delta_{U}^{1}: C^{1}(\pi, U)_{G} \rightarrow C^{2}(\pi, U)_{G}$ is given by

$$
\left(\delta_{U}^{1} s\right)(w, v, u):=s(v, u) s(w, u)^{-1} s(w, v)
$$

for $(w, v, u) \in V\left(E^{2}\left\lceil_{U}\right)\right.$. We call $\delta_{U}^{1} s$ the curvature of $s \in C^{1}(\pi, U)_{G}$.

Let $I$ be a pseudodirected set and $\left(U_{i}\right)_{i \in I}$ a system of subsets of $X^{2}$ such that for any $i, j \in I, \Delta_{X} \subset U_{i}$, and if $i \leq j$, then $U_{j} \subset U_{i}$. We can see that a map $\lim _{\longrightarrow} \delta_{U_{i}}^{1}: \lim _{\longrightarrow} C^{1}\left(\pi, U_{i}\right)_{G} \rightarrow \lim _{\rightarrow} C^{2}\left(\pi, U_{i}\right)_{G}$ is defined. We call $\left(\lim _{\longrightarrow} \delta_{U_{i}}^{1}\right)\left([s]_{I}\right)$ the curvature of $[s]_{I} \in \lim _{\rightarrow} C^{1}\left(\pi, U_{i}\right)_{G}$. In particular, $\left(\lim _{\longrightarrow} \delta_{U}^{1}\right)\left([s]_{\mathscr{N}_{X^{2}}\left(\Delta_{X}\right)}\right)$ is the curvature of $[s]_{\mathscr{N}_{X^{2}}\left(\Delta_{X}\right)} \in \lim _{\longrightarrow} C^{1}(\pi, U)_{G}$ in the sense of Asada $([4,5])$.

Put

$$
V^{u}\left(E^{2} \Upsilon_{U}\right):=\left\{\left(u_{1}, u_{0}, u_{2}\right) \in V\left(E^{2} \Upsilon_{U}\right) \mid u_{0}=u\right\} .
$$

We denote by $C^{2, u}(\pi, U)_{G}$ the set of continuous maps $f: V^{u}\left(E^{2}\left\lceil_{U}\right) \rightarrow G\right.$ such that

(1) $f\left(u_{1}, u, u_{2}\right)=1_{G}$ if $u_{1}=u$ or $u_{2}=u$ for $\left(u_{1}, u, u_{2}\right) \in V^{u}\left(E^{2}\left\lceil_{U}\right)\right.$,

(2) $f\left(u_{1} a, u b, u_{2} c\right)=b^{-1} f\left(u_{1}, u, u_{2}\right) b$ for $\left(u_{1}, u, u_{2}\right) \in V^{u}\left(E^{2}\left\lceil_{U}\right)\right.$ and $a, b, c \in G$.

A map $\varepsilon: C^{2}(\pi, U)_{G} \rightarrow C^{2, u}(\pi, U)_{G}$ is given by

$$
\varepsilon(f):=f \circ i_{V^{u}\left(E^{2} \Gamma_{U}\right), V\left(E^{2} \Gamma_{U}\right)}
$$

for $f \in C^{2}(\pi, U)_{G}$. Let $C_{\text {inv }}^{2}(\pi, U)_{G}$ denote the set of $f \in C^{2}(\pi, U)_{G}$ such that for $(u, v, w) \in V\left(E^{2}\left\lceil_{U}\right), f(u, v, w)=1_{G}\right.$ if $u=w$, and $f(u, v, w)=f(w, v, u)^{-1}$. There is a one-to-one correspondence between $C_{\text {inv }}^{2}(\pi, U)_{G}$ and $\mathscr{R}(\pi,\langle U\rangle)_{G}$. Two maps $v: C_{\text {inv }}^{2}(\pi, U)_{G} \rightarrow \mathscr{R}(\pi,\langle U\rangle)_{G}$ and $\lambda: \mathscr{R}(\pi,\langle U\rangle)_{G} \rightarrow C_{\text {inv }}^{2}(\pi, U)_{G}$ are defined by

$$
v(f)\left(\left(x_{0}, x_{2}, x_{1}, x_{0}\right), u_{0}\right):=u_{0} f\left(u_{1}, u_{0}, u_{2}\right)
$$

for $\left(\left(x_{0}, x_{2}, x_{1}, x_{0}\right), u_{0}\right) \in\left(\left.p_{0}\right|_{\left(\langle U\rangle_{\Delta_{X}}\right)_{(3)}}\right)^{*} E$ and $u_{i} \in E_{x_{i}}$ for $i \in\{1,2\}$, and

$$
\lambda\left(f^{\prime}\right)\left(u_{1}, u_{0}, u_{2}\right):=T\left(u_{0}, f^{\prime}\left(\left(\pi\left(u_{0}\right), \pi\left(u_{2}\right), \pi\left(u_{1}\right), \pi\left(u_{0}\right)\right), u_{0}\right)\right)
$$

for $\left(u_{1}, u_{0}, u_{2}\right) \in V\left(E^{2}\left\lceil_{U}\right)\right.$. We can see that $\lambda=v^{-1}$. For the maps $v_{i}: C_{\text {inv }}^{2}\left(\pi, U_{i}\right)_{G} \rightarrow \mathscr{R}\left(\pi,\left\langle U_{i}\right\rangle\right)_{G}$ and $\lambda_{i}: \mathscr{R}\left(\pi,\left\langle U_{i}\right\rangle\right)_{G} \rightarrow C_{\text {inv }}^{2}\left(\pi, U_{i}\right)_{G}$, we obtain the equality $\lim _{i} \lambda_{i}=\left(\lim _{\longrightarrow} v_{i}\right)^{-1}$. 
Proposition 5.6. For

$$
\left.[s]_{I} \in \lim _{\longrightarrow} C_{\mathrm{inv}}^{1}\left(\pi, U_{i}\right)_{G}, \quad \Phi_{I}^{u, 1}\left(\left[P^{\omega^{s}}\right]_{I}\right)=\Omega^{u}\left(\left(\lim _{\longrightarrow} v_{i} \circ \delta_{U_{i}}^{1}\right)\left([s]_{I}\right)\right)\right) .
$$

In particular, $\Phi_{\mathcal{N}_{X^{2}}\left(\Delta_{X}\right)}^{u, 1}\left(\left[P^{\omega^{s}}\right]_{\mathscr{N}_{X^{2}}\left(\Delta_{X}\right)}\right)=\Omega^{u}\left(\left(\lim _{\longrightarrow} v_{U} \circ \delta_{U}^{1}\right)\left([s]_{\mathscr{N}_{X^{2}}\left(\Delta_{X}\right)}\right)\right)$ for $[s]_{\mathscr{N}_{X^{2}}\left(\Delta_{X}\right)}$ $\in \lim _{\longrightarrow} C_{\text {inv }}^{1}(\pi, U)_{G}$.

Proof. It suffices to show that for $s \in C_{\text {inv }}^{1}(\pi, U)_{G}, R^{P^{\omega^{s}}}=v\left(\delta_{U}^{1} s\right)$. We have

$$
\begin{aligned}
R^{P^{\omega^{s}}}\left(\left(x_{0}, x_{2}, x_{1}, x_{0}\right), u_{0}\right) & =P^{\omega^{s}}\left(\left(x_{0}, x_{2}, x_{1}, x_{0}\right), u_{0}\right) \\
& =\omega_{x_{0}, x_{2}}^{s}\left(\omega_{x_{2}, x_{1}}^{s}\left(\omega_{x_{1}, x_{0}}^{s}\left(u_{0}\right)\right)\right)=u_{0} s\left(u_{0}, u_{2} s\left(u_{2}, u_{1} s\left(u_{1}, u_{0}\right)\right)\right) \\
& =u_{0} s\left(u_{0}, u_{2}\right) s\left(u_{1}, u_{2}\right)^{-1} s\left(u_{1}, u_{0}\right)=v\left(\delta_{U}^{1} s\right)\left(x_{0}, x_{2}, x_{1}, x_{0}\right)
\end{aligned}
$$

for $\left(\left(x_{0}, x_{2}, x_{1}, x_{0}\right), u_{0}\right) \in\left(\left.p_{0}\right|_{\left(\langle U\rangle_{\Delta_{X}}\right)_{(3)}}\right)^{*} E$ and $u_{i} \in E_{x_{i}}$ for $i \in\{1,2\}$.

Let $\pi: E \rightarrow X$ be a smooth principal $G$-bundle, $X$ a Riemannian manifold, and $H$ a connection in $E$. We denote by $\Psi_{\text {loc }}^{u}(H)$ the local holonomy group of $H$, that is, $\Psi_{\mathrm{loc}}^{u}(H)=\bigcap_{U} \Psi^{u, 0}\left(\left.H\right|_{U}\right)$, where $U$ runs through all connected open neighborhoods of $\pi(u),\left.H\right|_{U}$ is the connection in $E\left\lceil_{U}\right.$ induced by $H$, and $\Psi^{u, 0}\left(\left.H\right|_{U}\right)$ is the restricted holonomy group of $\left.H\right|_{U}$ with reference point $u$.

Proposition 5.7. Under the assumption of Proposition 5.2, we have $\Psi_{\text {loc }}^{u}(H) \cap \Psi_{\text {geo }}^{u}(H)=\Phi_{\mathscr{S} \mathscr{C}(X)}^{u, 1}\left(\left[P^{\omega^{H}}\right]_{\mathscr{S} \mathscr{C}(X)}\right)$ for $\omega^{H} \in S F_{\text {inv }}\left(\pi, U_{\mathscr{V}}\right)_{G}$.

Proof. Let $a \in \Phi_{\mathscr{\mathscr { C }} \mathscr{C}(X)}^{u, 1}\left(\left[P^{\omega^{H}}\right]_{\mathscr{S} \mathscr{C}(X)}\right)$ and $W$ be a connected open neighborhood of $\pi(u)$. Let $\mathscr{V}^{\prime}$ be the open covering of $X \backslash\{\pi(u)\}$ consists of all strongly convex sets contained in any element of $\mathscr{V}$. On the other hand, let $V \in \mathscr{V}$ with $\pi(u) \in V$ and $C$ be an open strongly convex set with $\pi(u) \in C \subset W \cap V$. Then $\mathscr{V}^{\prime \prime}:=\mathscr{V}^{\prime} \cup\{C\}$ is an open covering of $X$ consists of strongly convex sets and a refinement of $\mathscr{V}$. In particular, we have $a \in \operatorname{Im}\left(\left.\left(P^{\omega^{H}}\right)^{u}\right|_{\left\langle U_{\mathcal{V}^{\prime \prime}}\right\rangle_{\pi(u)}^{1}}\right)$. Then, by a similar argument as in the proof of Proposition 5.2, there exists $\left(x_{n}, \ldots, x_{1}, x_{0}\right) \in$ $\left\langle U_{\mathscr{V}^{\prime \prime}}\right\rangle_{\pi(u)}^{1}$ and a unique family of shortest geodesics $\left(\gamma_{k}:[0,1] \rightarrow X\right)_{k \in\{0, \ldots, n-1\}}$ such that $\gamma_{k}(0)=x_{k}$ and $\gamma_{k}(1)=x_{k+1}$, and

$$
a=\left(P^{\omega^{H}}\right)^{u}\left(x_{n}, \ldots, x_{1}, x_{0}\right)=T\left(u, \tau_{\gamma_{n-1} \cdots \gamma_{1} \gamma_{0}}^{H}(u)\right) .
$$

Since $\left(x_{k}, \pi(u)\right) \in U_{\mathscr{\gamma}^{\prime \prime}}$ for any $k \in\{1, \ldots, n-1\}$, we get $x_{k} \in C$ and $\operatorname{Im} \gamma_{k} \subset$ $C \subset W$ for any $k \in\{0, \ldots, n-1\}$. Note that the curve $\gamma_{n-1} \cdots \gamma_{1} \gamma_{0}$ is homotopic to the point $\pi(u)$. Therefore, $a \in \Psi^{u, 0}\left(\left.H\right|_{W}\right) \cap \Psi_{\text {geo }}^{u}(H)$, that is, $\Phi_{\mathscr{S} \mathscr{C}(X)}^{u, 1}\left(\left[P^{\omega^{H}}\right]_{\mathscr{C} \mathscr{C}(X)}\right)$ $\subset \Psi_{\mathrm{loc}}^{u}(H) \cap \Psi_{\mathrm{geo}}^{u}(H)$. 
Conversely, let $a \in \Psi_{\text {loc }}^{u}(H) \cap \Psi_{\mathrm{geo}}^{u}(H)$ and $f \in\left[\left(P^{\omega^{H}}\right)^{u}\right]_{\mathscr{S} \mathscr{C}(X)}$. Then, there exists $\mathscr{W} \in \mathscr{S} \mathscr{C}(X)$ such that $\left.f\right|_{\left\langle U_{\mathscr{W}}\right\rangle_{\pi(u)}^{1}}=\left.\left(P^{\omega^{H}}\right)^{u}\right|_{\left\langle U_{\mathscr{W}}\right\rangle_{\pi(u)}^{1}}$. Put $W^{\prime}:=\bigcup\{V \in \mathscr{W} \mid$ $\pi(u) \in V\}$. Since $W^{\prime}$ is connected, we get $a \in \Psi^{u, 0}\left(\left.H\right|_{W^{\prime}}\right) \cap \Psi_{\text {geo }}^{u}(H)$. Then, there exists a family of geodesics $\left(\gamma_{k}:[0,1] \rightarrow W^{\prime}\right)_{k \in\{1, \ldots, n\}}$ such that $a=$ $T\left(u, \tau_{\gamma_{n} \cdots \gamma_{1}}^{H}(u)\right)$. By a similar argument as in the proof of Proposition 5.2, for any $k \in\{1, \ldots, n\}$, there exists a partition $0=t_{0}^{k}<t_{1}^{k}<\cdots<t_{m_{k}}^{k}=1$ of $[0,1]$ such that $\gamma_{k}\left(\left[t_{l-1}^{k}, t_{l}^{k}\right]\right)$ is contained in some $V_{l} \in \mathscr{W}$ with $\pi(u) \in V_{l}$ for $l \in\left\{1, \ldots, m_{k}\right\}$, and

$$
\tau_{\gamma_{n} \cdots \gamma_{1}}^{H}=P_{\left(\gamma_{n}(1), \gamma_{n}\left(t_{m_{n}-1}^{n}\right), \ldots, \gamma_{1}\left(t_{1}^{1}\right), \gamma_{1}(0)\right)}^{\omega^{H}}
$$

Then we have

$$
\begin{aligned}
a & =T\left(u, \tau_{\gamma_{n} \cdots \gamma_{1}}^{H}(u)\right) \\
& =\left(P^{\omega^{H}}\right)^{u}\left(\gamma_{n}(1), \gamma_{n}\left(t_{m_{n}-1}^{n}\right), \ldots, \gamma_{1}\left(t_{1}^{1}\right), \gamma_{1}(0)\right) \in \operatorname{Im}\left(\left.\left(P^{\omega^{H}}\right)^{u}\right|_{\left\langle U_{\mathscr{W}}\right\rangle_{\pi(u)}^{1}}\right),
\end{aligned}
$$

that is, $\quad a \in \operatorname{Im}\left(\left.f\right|_{\left\langle U_{\mathscr{W}}\right\rangle_{\pi(u)}^{1}}\right) \subset \operatorname{Im} f . \quad$ Therefore, $\quad \Psi_{\text {loc }}^{u}(H) \cap \Psi_{\text {geo }}^{u}(H) \subset$ $\Phi_{\mathscr{S} \mathscr{C}(X)}^{u, 1}\left(\left[P^{\omega^{H}}\right]_{\mathscr{S} \mathscr{C}(X)}\right)$.

\section{A Holonomy Reduction Theorem and Related Results}

In this section, we define a holonomy bundle of the parallel displacement and study its fundamental properties. As in the smooth category and [1], we obtain a holonomy reduction theorem and related results. In order to clarify the arguments, we introduce a category $\mathbf{C}$, whose objects are quadruples $(\pi, G, P, u)$, where $\pi$ is a principal $G$-bundle, $P \in \mathscr{P} \mathscr{D}(\pi, \subseteq)_{G}$ is a parallel displacement, and $u \in E=\operatorname{dom} \pi$.

Let $\pi: E \rightarrow X$ be a principal $G$-bundle, $P \in \mathscr{P} \mathscr{D}(\pi, \subseteq)_{G}$, and $u \in E$.

Definition 6.1. Put

$$
E^{u}:=E^{u}(P):=\left\{P(\mathbf{x}, u) \mid \mathbf{x} \in \Im_{X \times\{\pi(u)\}}\right\} .
$$

The bundle $\pi^{u}:=\left.\pi\right|_{E^{u}}: E^{u} \rightarrow X$ is called the holonomy bundle of $P$ through $u$. We assume that the topology of $E^{u}$ is the relative topology induced from $E$.

Note that if $X$ is $\Xi$-connected, then $\pi^{u}$ is surjective. Similar to the holonomy bundle of a connection in the smooth category, the following fundamental properties hold. 
Proposition 6.1. (i) For $u, v \in E$ and $\mathbf{x} \in \mathfrak{S}$ such that $v=P(\mathbf{x}, u)$, we have $E^{u}=E^{v}$.

(ii) For $(u, a) \in E \times G, E^{u a}=r_{a}\left(E^{u}\right)$, where $r_{a}: E \rightarrow E$ is a map assigning va to $v$.

(iii) If $X$ is S-connected, $E=\bigcup_{a \in G} E^{u a}$ for $u \in E$.

Proof. For $w=P(\mathbf{y}, v) \in E^{v}$, since $w=P(\mathbf{y} \bullet \mathbf{x}, u) \in E^{u}$, we have $E^{v} \subset E^{u}$. Similarly, we have $E^{u} \subset E^{v}$ and obtain (i). For $w=P(\mathbf{y}, u a) \in E^{u a}$, since $w=$ $P(\mathbf{y}, u) a \in r_{a}\left(E^{u}\right)$, we have $E^{u a} \subset r_{a}\left(E^{u}\right)$. Similarly, we have $r_{a}\left(E^{u}\right) \subset E^{u a}$ and obtain (ii). For $v \in E$ and $\mathbf{x} \in \mathfrak{\Xi}_{\pi(v), \pi(u)}$, since

$$
v=P(\mathbf{x}, u) T(P(\mathbf{x}, u), v)=P(\mathbf{x}, u T(P(\mathbf{x}, u), v)),
$$

we have $v \in E^{u a}$, where $a:=T(P(\mathbf{x}, u), v)$. Since $E^{u a} \subset E$ for any $a \in G$, we have $\bigcup_{a \in G} E^{u a} \subset E$ and obtain (iii).

Let $\pi: E \rightarrow X$ (resp. $\left.\pi^{\prime}: E^{\prime} \rightarrow X\right)$ be a $G$ (resp. $G^{\prime}$ )-bundle and $(h, \rho)$ : $\left(\pi^{\prime}, G^{\prime}\right) \rightarrow(\pi, G)$ a homomorphism. We call $(h, \rho)$ a reduction of $\pi$ to $\pi^{\prime}$ if $h$ and $\rho$ are injective. Let $(h, \rho)$ be a reduction of $\pi$ to $\pi^{\prime}$. For $\left.P \in \mathscr{P} \mathscr{D}(\pi, \subseteq)\right)_{G}$ and $P^{\prime} \in \mathscr{P} \mathscr{D}_{G^{\prime}}\left(\pi^{\prime}, \mathfrak{S}\right)$, if $(h, \rho)$ preserves $P^{\prime}$ and $P$, we say that $P$ reduces to $P^{\prime}$ and call $P^{\prime}$ a reduced parallel displacement of $P$. For clarity, we introduce the following category C. For a principal $G$-bundle $\pi: E \rightarrow X, P \in \mathscr{P} \mathscr{D}(\pi, \subseteq)_{G}$, and $u \in E$, we consider a quadruple $(\pi, G, P, u)$ as an object in $\mathbf{C}$. We denote by $\mathbf{C}_{0}$ the collection of objects in $\mathbf{C}$. A morphism between $(\pi, G, P, u)$ and $\left(\pi^{\prime}, G^{\prime}, P^{\prime}, u^{\prime}\right)$ in $\mathbf{C}$ is a homomorphism $(h, f, \rho):(\pi, G) \rightarrow\left(\pi^{\prime}, G^{\prime}\right)$ preserving $P$ and $P^{\prime}$, and satisfying $h(u)=u^{\prime}$. For simplicity, we denote by $P\left\lceil_{E^{u}}\right.$ the restriction $E^{u}|P|_{\left(p_{0} \mid \Xi\right)^{*} E^{u}}$. From the property of the relative topology, $\Phi^{u}|T|_{\left(E^{u}\right)^{*}}$ is continuous. Thus, once $\pi^{u}$ is proved to be a $\Phi^{u}$-bundle, it is a principal $\Phi^{u}$-bundle. Again, from the property of the relative topology, $\left(i_{E^{u}, E}, i_{\Phi^{u}, G}\right)$ is a reduction of $\pi$ to $\pi^{u}$ and $P\left\lceil_{E^{u}} \in \mathscr{P} \mathscr{D}_{\Phi^{u}}\left(\pi^{u}, \subseteq\right)\right.$ is a reduced parallel displacement of $P$. Then, we define a holonomy reduction of $(\pi, G, P, u) \in \mathbf{C}_{0}$.

Definition 6.2. For $u \in E$, we call a quadruple $\left(\pi^{u}, \Phi^{u}, P\left\lceil_{E^{u}}, u\right)\right.$ the holonomy reduction of $(\pi, G, P, u)$ if $\left(\pi^{u}, \Phi^{u}, P\left\lceil_{E^{u}}, u\right) \in \mathbf{C}_{0}\right.$.

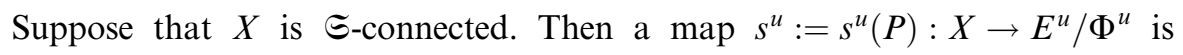
defined as follows. For $x \in X$, choose $\mathbf{x} \in \Xi_{x, \pi(u)}$ and put

$$
s^{u}(x):=[P(\mathbf{x}, u)] .
$$


This definition does not depend on the choice of $\mathbf{x}$ as follows. For any $\mathbf{y} \in \mathfrak{\Xi}_{x, \pi(u)}$, since

$$
P(\mathbf{y}, u)=P\left(\mathbf{x}, u T\left(u, P\left(\mathbf{x}^{-} \bullet \mathbf{y}, u\right)\right)\right)=P(\mathbf{x}, u) P^{u}\left(\mathbf{x}^{-} \bullet \mathbf{y}\right),
$$

we have $[P(\mathbf{y}, u)]=[P(\mathbf{x}, u)]$. Note that $\pi^{u} /^{\Phi^{u}}$ (see Section 2) is well-defined. Since

$$
\pi^{u} /^{\Phi^{u}}\left(s^{u}(x)\right)=\pi^{u} /^{\Phi^{u}}([P(\mathbf{x}, u)])=\pi^{u}(P(\mathbf{x}, u))=x
$$

for $x \in X$ and

$$
s^{u}\left(\left.\pi^{u}\right|^{\Phi^{u}}([P(\mathbf{x}, u)])\right)=s^{u}\left(\pi^{u}(P(\mathbf{x}, u))\right)=s^{u}\left(\left(p_{\infty} \mid \Subset\right)(\mathbf{x})\right)=[P(\mathbf{x}, u)]
$$

for $[P(\mathbf{x}, u)] \in E^{u} / \Phi^{u}$, we have $s^{u}=\left(\pi^{u} / \Phi^{u}\right)^{-1}$. From Lemma 2.3, $\pi^{u}$ is a $\Phi^{u}$ bundle if and only if $\pi^{u} / \Phi^{u}$ is a homeomorphism. Thus, by the Definition 6.2, we have the following proposition.

Proposition 6.2. For $u \in E,\left(\pi^{u}, \Phi^{u}, P \Gamma_{E^{u}}, u\right)$ is the holonomy reduction of $(\pi, G, P, u) \in \mathbf{C}_{0}$ if and only if $s^{u}$ is continuous.

We have a sufficient condition for an object to admit the holonomy reduction as follows.

THeOREM 6.3 (Holonomy Reduction Theorem). Let $(\pi, G, P, u) \in \mathbf{C}_{0}$, where $\pi: E \rightarrow X$ is a principal $G$-bundle, $P \in \mathscr{P} \mathscr{D}(\pi, \mathfrak{S})_{G}$ is a parallel displacement, and

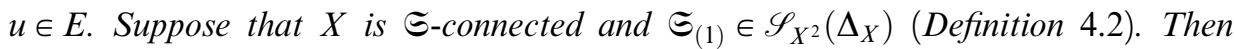
$\left(\pi^{u}, \Phi^{u}, P \Gamma_{E^{u}}, u\right)$ is the holonomy reduction of $(\pi, G, P, u)$. Moreover, $\pi^{u}$ is a locally trivial $\Phi^{u}$-bundle.

Proof. Note that the diagram

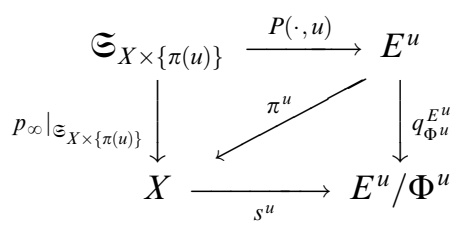

commutes. From Proposition 4.2, $\left.p_{\infty}\right|_{\varsigma_{X \times\{\pi(u)\}}}$ is an identification. Then, from upper half of the diagram, $\pi^{u}$ is an identification. Again, from lower half of the 
diagram, $s^{u}$ is continuous. Thus from Proposition 6.2, $\left(\pi^{u}, \Phi^{u}, P\left\lceil_{E^{u}}, u\right)\right.$ is the holonomy reduction of $(\pi, G, P, u)$. Then, from Theorem 4.4 (ii), $\pi^{u}$ is a locally trivial $\Phi^{u}$-bundle.

Suppose that two objects both admit the holonomy reduction defined in Definition 6.2. Then a bundle homomorphism between holonomy bundles which preserves the structure involved induces a continuous group homomorphism between holonomy groups. Moreover, we obtain a relation between $\operatorname{Ker} P^{u}$ and Ker $P^{\prime u^{\prime}}$.

Proposition 6.4. Let $(\pi, G, P, u)\left(\right.$ resp. $\left.\left(\pi^{\prime}, G^{\prime}, P^{\prime}, u^{\prime}\right)\right) \in \mathbf{C}_{0}$, where $\pi: E \rightarrow X$ (resp. $\left.\pi^{\prime}: E^{\prime} \rightarrow X^{\prime}\right)$ is a principal $G$ (resp. $\left.G^{\prime}\right)$-bundle, $P \in \mathscr{P} \mathscr{D}(\pi, \subseteq)_{G}$ (resp. $\left.P^{\prime} \in \mathscr{P} \mathscr{D}\left(\pi^{\prime}, \mathfrak{S}^{\prime}\right)_{G^{\prime}}\right)$ is a parallel displacement, and $u \in E$ (resp. $\left.u^{\prime} \in E^{\prime}\right)$. Suppose that $X$ (resp. $\left.X^{\prime}\right)$ is $\subseteq$ (resp. $\left.\mathfrak{S}^{\prime}\right)$-connected and $\left(\pi^{u}, \Phi^{u}, P\left\lceil_{E^{u}}, u\right)\right.$ (resp. $\left(\pi^{\prime u^{\prime}}, \Phi^{u^{\prime}}\right.$, $\left.P^{\prime}\left\lceil_{E^{\prime u^{\prime}}}, u^{\prime}\right)\right)$ is the holonomy reduction of $(\pi, G, P, u)$ (resp. $\left.\left(\pi^{\prime}, G^{\prime}, P^{\prime}, u^{\prime}\right)\right)$. Let $f: X \rightarrow X^{\prime}$ be a continuous map preserving $\mathfrak{\subseteq}$ and $\mathfrak{S}^{\prime}$, and satisfying $f(\pi(u))=$ $\pi^{\prime}\left(u^{\prime}\right)$. Then we have implications (i) $\Rightarrow$ (ii) and (ii) $\Rightarrow$ (iii):

(i) There exists a bundle morphism $\left(h^{u^{\prime} u}, f\right): \pi^{u} \rightarrow \pi^{\prime u^{\prime}}$ preserving $P\left\lceil_{E^{u}}\right.$ and $P^{\prime} \Gamma_{E^{\prime u^{\prime}}}$, and satisfying $h^{u^{\prime} u}(u)=u^{\prime}$.

(ii) There exists a continuous group homomorphism $\rho^{u^{\prime} u}: \Phi^{u} \rightarrow \Phi^{u^{\prime}}$ such that $\rho^{u^{\prime} u} \circ P^{u}=P^{\prime u^{\prime}} \circ f^{\sqcup}$.

(iii) $f^{\sqcup}\left(\operatorname{Ker} P^{u}\right) \subset \operatorname{Ker} P^{\prime u^{\prime}}$.

Proof. Firstly, we show the implication (i) $\Rightarrow\left(\right.$ ii). Put $\rho^{u^{\prime} u}:=T^{\prime}\left(u^{\prime}, \cdot\right) \circ$ $h^{u^{\prime} u} \circ l_{u}: \Phi^{u} \rightarrow \Phi^{u^{\prime}}$, where $T^{\prime}$ is the continuous translation function of $\pi^{\prime u^{\prime}}$. Then we can see that $\rho^{u^{\prime} u}$ satisfies required condition.

Secondly, we show the implication (ii) $\Rightarrow$ (iii). Let $\mathbf{x} \in \operatorname{Ker} P^{u}$. Since $P^{u}(\mathbf{x})=1_{G}$,

$$
P^{\prime u^{\prime}}\left(f^{\sqcup}(\mathbf{x})\right)=\rho^{u^{\prime} u}\left(P^{u}(\mathbf{x})\right)=\rho^{u^{\prime} u}\left(1_{\Phi^{u}}\right)=1_{\Phi^{u^{\prime}}} .
$$

Thus $f^{\sqcup}(\mathbf{x}) \in \operatorname{Ker} P^{\prime u^{\prime}}$.

Note that the bundle morphism (resp. continuous group homomorphism) satisfying the condition in (i) (resp. (ii)) is unique. For $b \in G$, let $A d_{b}$ be the inner automorphism of $G$ given by $A d_{b}(c):=b c b^{-1}$ for $c \in G$.

Corollary 6.5. For $a^{\prime} \in G^{\prime}$, we have $\rho^{\left(u^{\prime} a^{\prime}\right) u}=A d_{a^{\prime-1}} \circ \rho^{u^{\prime} u}$. 
Proof. For $b=P^{u}(\mathbf{x}) \in \Phi^{u}$, since

$$
\begin{aligned}
\rho^{\left(u^{\prime} a^{\prime}\right) u}(b) & =\rho^{\left(u^{\prime} a^{\prime}\right) u}\left(P^{u}(\mathbf{x})\right)=P^{\prime u^{\prime} a}\left(f^{\sqcup}(\mathbf{x})\right)=T^{\prime}\left(u^{\prime} a^{\prime}, P^{\prime}\left(f^{\sqcup}(\mathbf{x}), u^{\prime} a^{\prime}\right)\right) \\
& =a^{\prime-1} T^{\prime}\left(u^{\prime}, P^{\prime}\left(f^{\sqcup}(\mathbf{x}), u^{\prime}\right)\right) a^{\prime}=\operatorname{Ad}_{a^{\prime-1}}\left(\rho^{u^{\prime} u}(b)\right),
\end{aligned}
$$

we have the conclusion.

The following proposition gives the canonical definition of the bundle homomorphism satisfying the condition (i) of Proposition 6.4.

Proposition 6.6. Let $(\pi, G, P, u)\left(\right.$ resp. $\left.\left(\pi^{\prime}, G^{\prime}, P^{\prime}, u^{\prime}\right)\right) \in \mathbf{C}_{0}$ and $f: X \rightarrow X^{\prime}$ be a continuous map preserving $\subseteq$ and $\mathfrak{\Xi}^{\prime}$, and satisfying $f(\pi(u))=\pi^{\prime}\left(u^{\prime}\right)$, where $\pi: E \rightarrow X\left(\right.$ resp. $\left.\pi^{\prime}: E^{\prime} \rightarrow X^{\prime}\right)$ is a principal $G\left(\right.$ resp. $\left.G^{\prime}\right)$-bundle, $P \in \mathscr{P} \mathscr{D}(\pi, \subseteq){ }_{G}$ (resp. $\left.P^{\prime} \in \mathscr{P} \mathscr{D}\left(\pi^{\prime}, \subseteq^{\prime}\right)_{G^{\prime}}\right)$ is a parallel displacement, and $u \in E$ (resp. $\left.u^{\prime} \in E^{\prime}\right)$. Suppose that $X$ is $\Xi$-connected and $f^{\sqcup}\left(\operatorname{Ker} P^{u}\right) \subset \operatorname{Ker} P^{\prime u^{\prime}}$. Then a map $h^{u^{\prime} u}$ : $E^{u} \rightarrow E^{\prime u^{\prime}}$ is defined by

$$
h^{u^{\prime} u}(v):=P^{\prime}\left(f^{\sqcup}(\mathbf{x}), u^{\prime}\right)
$$

for $v=P(\mathbf{x}, u) \in E^{u}$. The map $h^{u^{\prime} u}$ satisfies the conditions $\pi^{\prime u^{\prime}} \circ h^{u^{\prime} u}=f \circ \pi^{u}$, $h^{u^{\prime} u} \circ P\left\lceil_{E^{u}}=P^{\prime}\left\lceil_{E^{\prime u^{\prime}}} \circ\left(f^{\sqcup} \times h^{u^{\prime} u}\right)\right.\right.$, and $h^{u^{\prime} u}(u)=u^{\prime}$. Moreover, every map satisfying these conditions coincide with $h^{u^{\prime} u}$.

Proof. The definition of $h^{u^{\prime} u}$ does not depend on the choice of $\mathbf{x}$ as is shown below. Let $\mathbf{y} \in \mathfrak{S}_{\pi(v) \pi(u)}$ be any sequence such that $v=P(\mathbf{y}, u)$. Since $P\left(\mathbf{y}^{-} \bullet \mathbf{x}, u\right)=u$, we have $P^{u}\left(\mathbf{y}^{-} \bullet \mathbf{x}\right)=1_{\Phi^{u}}$. By the assumption, since $f^{\sqcup}\left(\right.$ Ker $\left.P^{u}\right)$ $\subset \operatorname{Ker} P^{\prime u^{\prime}}$, we have $P^{\prime u^{\prime}}\left(f^{\sqcup}\left(\mathbf{y}^{-} \bullet \mathbf{x}\right)\right)=1_{\Phi^{u^{\prime}}}$. Then $P^{\prime}\left(f^{\sqcup}(\mathbf{x}), u^{\prime}\right)=P^{\prime}\left(f^{\sqcup}(\mathbf{y}), u^{\prime}\right)$. We can see that $h^{u^{\prime} u}$ satisfies the required conditions. Let $k: E^{u} \rightarrow E^{\prime u^{\prime}}$ be any map satisfying the required conditions. For $v=P(\mathbf{x}, u) \in E^{u}$, since

$$
k(v)=k\left(P\left\lceil_{E^{u}}(\mathbf{x}, u)\right)=P^{\prime}\left\lceil_{E^{\prime u^{\prime}}}\left(f^{\sqcup}(\mathbf{x}), k(u)\right)=P^{\prime}\left\lceil_{E^{\prime u^{\prime}}}\left(f^{\sqcup}(\mathbf{x}), u^{\prime}\right)=h^{u^{\prime} u}(v),\right.\right.\right.
$$

we have $k=h^{u^{\prime} u}$.

In this proposition, we do not require that $\left(\pi^{u}, \Phi^{u}, P\left\lceil_{E^{u}}, u\right)\right.$ (resp. $\left(\pi^{\prime u^{\prime}}, \Phi^{u^{\prime}}\right.$, $\left.\left.P^{\prime} \Gamma_{E^{\prime u^{\prime}}}, u^{\prime}\right)\right)$ is the holonomy reduction of $(\pi, G, P, u)\left(\operatorname{resp} .\left(\pi^{\prime}, G^{\prime}, P^{\prime}, u^{\prime}\right)\right)$ or $h^{u^{\prime} u}$ is continuous. Under an appropriate condition, we have the implication (ii) $\Rightarrow$ (i) in Proposition 6.4 .

Proposition 6.7. Under the assumption of Proposition 6.4, suppose further that $X$ (resp. $\left.X^{\prime}\right)$ is $\mathfrak{\subseteq}$-connected (resp. $\mathfrak{S}^{\prime}$-connected), $\mathfrak{S}_{(1)} \in \mathscr{S}_{X^{2}}\left(\Delta_{X}\right)$, and 
$\mathfrak{S}_{(1)}^{\prime} \in \mathscr{T}_{X^{\prime 2}}\left(\Delta_{X^{\prime}}\right)$. Then for each continuous map $f: X \rightarrow X^{\prime}$ preserving $\mathfrak{\subseteq}$ and $\mathfrak{\Xi}^{\prime}$, and satisfying $f(\pi(u))=\pi^{\prime}\left(u^{\prime}\right)$, conditions (i) and (ii) in Proposition 6.4 are equivalent.

Proof. From Theorem 6.3, $\left(\pi^{u}, \Phi^{u}, P\left\lceil_{E^{u}}, u\right)\right.$ (resp. $\left(\pi^{\prime u^{\prime}}, \Phi^{u^{\prime}}, P^{\prime}\left\lceil_{E^{\prime u^{\prime}}}, u^{\prime}\right)\right)$ is holonomy reduction of $(\pi, G, P, u)$ (resp. $\left.\left(\pi^{\prime}, G^{\prime}, P^{\prime}, u^{\prime}\right)\right)$. Thus from Proposition 6.4, (i) implies (ii). Conversely, suppose that (ii) holds. Again from Proposition 6.4, we have $f^{\sqcup}\left(\operatorname{Ker} P^{u}\right) \subset \operatorname{Ker} P^{\prime u^{\prime}}$. Let $h^{u^{\prime} u}: E^{u} \rightarrow E^{\prime u^{\prime}}$ be the map defined in Proposition 6.6. Let $x \in X$ and $V_{x}$ be an open neighborhood of $x$ in $X$ such that $V_{x} \times\{x\} \subset \mathfrak{S}_{(1)}$. Fix $c_{x} \in \mathfrak{S}_{x, \pi(u)}$ and let $\alpha_{x}: E\left\lceil_{V_{x}} \rightarrow V_{x} \times G\right.$ be a local trivialization defined in the proof of Theorem 4.4 (ii). Moreover, let $V_{f(x)}^{\prime}$ be an open neighborhood of $f(x)$ in $X^{\prime}$ such that $V_{f(x)}^{\prime} \times\{f(x)\} \subset \mathfrak{S}_{(1)}^{\prime}$. Put $c_{f(x)}^{\prime}:=f^{\sqcup}\left(c_{x}\right) \in$ $\mathfrak{\subseteq}_{f(x), f(\pi(u))}^{\prime}$ and let $\alpha_{f(x)}^{\prime}: E^{\prime}\left\lceil_{V_{f(x)}^{\prime}} \rightarrow V_{f(x)}^{\prime} \times G^{\prime}\right.$ be a local trivialization defined in the proof of Theorem 4.4 (ii). Then, for $v=P(\mathbf{x}, u) \in E^{u}\left\lceil_{V_{x} \cap f^{-1}\left(V_{f(x)}^{\prime}\right)}\right.$, we get

$$
\begin{aligned}
\left(\alpha_{f(x)}^{\prime-1}\right. & \left.\circ\left(f \times \rho^{u^{\prime} u}\right) \circ \alpha_{x}\right)(v) \\
& =P^{\prime}\left((f(\pi(v)), f(x)) \bullet c_{f(x)}^{\prime}, l_{u^{\prime}}\left(\rho^{u^{\prime} u}\left(T\left(u, P\left(c_{x}^{-} \bullet(x, \pi(v)), v\right)\right)\right)\right)\right) \\
& =P^{\prime}\left((f(\pi(v)), f(x)) \bullet c_{f(x)}^{\prime}, h^{u^{\prime} u}\left(P\left(c_{x}^{-} \bullet(x, \pi(v)) \bullet \mathbf{x}, u\right)\right)\right) \\
& =P^{\prime}\left(f^{\sqcup}\left((\pi(v), x) \bullet c_{x}\right), P^{\prime}\left(f^{\sqcup}\left(c_{x}^{-} \bullet(x, \pi(v)) \bullet \mathbf{x}, u^{\prime}\right)\right)\right) \\
& =P^{\prime}\left(f^{\sqcup}(\mathbf{x}), u^{\prime}\right)=h^{u^{\prime} u}(v) .
\end{aligned}
$$

Thus, $h^{u^{\prime} u}$ is continuous.

COROLlary 6.8. Under the assumption of Proposition 6.7, suppose that there exists a continuous group homomorphism $\rho^{u^{\prime} u}: \Phi^{u} \rightarrow \Phi^{u^{\prime}}$ such that $\rho^{u^{\prime} u} \circ P^{u}=$ $P^{\prime u^{\prime}} \circ f^{\sqcup}$. Then $\left(h^{u^{\prime} u}, f, \rho^{u^{\prime} u}\right):\left(\pi^{u}, \Phi^{u}, P\left\lceil_{E^{u}}, u\right) \rightarrow\left(\pi^{\prime u^{\prime}}, \Phi^{u^{\prime}}, P^{\prime}\left\lceil_{E^{\prime u^{\prime}}}, u^{\prime}\right)\right.\right.$ is a morphism in $\mathbf{C}$.

\section{Strong Holonomy Reduction}

In this section, we introduce a concept of strong holonomy reduction and obtain further results applied in the succeeding section. In Proposition 6.6, if $f^{\sqcup}\left(\operatorname{Ker} P^{u}\right) \subset \operatorname{Ker} P^{\prime u^{\prime}}$, the map $h^{u^{\prime} u}: E^{u} \rightarrow E^{\prime u^{\prime}}$ satisfying the required conditions is uniquely defined. Recall that $h^{u^{\prime} u}$ is not necessarily continuous in general. For the continuity of $h^{u^{\prime} u}$, we introduce the identification topology to holonomy groups and holonomy bundles, while $\Phi^{u}$ is no longer a topological 
group in general. For this reason, we consider sufficient conditions for $\Phi^{u}$ to be a topological group with respect to the identification topology. We begin with the definition of strong holonomy groups and bundles.

Definition 7.1. Let $\pi: E \rightarrow X$ be a principal $G$-bundle, $P \in \mathscr{P} \mathscr{D}(\pi, \subseteq)_{G}$, and $u \in E$. We call the subgroup $\Phi^{u}=P^{u}\left(\Im_{\pi(u)}\right)$ of $G$ the strong holonomy group of $P$ with reference point $u$ if it is endowed with the identification topology induced by $\Phi^{u} \mid P^{u}$. We call the subbundle $\pi^{u}: E^{u} \rightarrow X$ the strong holonomy bundle through $u$ if the topology of $E^{u}$ is the identification topology induced by $E^{u} \mid P(\cdot, u)$.

Note that the binary operation of $\Phi^{u}$ is not necessarily continuous whereas unary operation is always continuous. Hereafter in this section, let $(\pi, G, P, u) \in$ $\mathbf{C}_{0}$, where $\pi$ is a principal $G$-bundle, $P \in \mathscr{P} \mathscr{D}(\pi, \Xi)_{G}$, and $u \in E$, and unless otherwise mentioned, we assume that $\Phi^{u}$ (resp. $\pi^{u}$ ) is the strong holonomy group (resp. bundle). Note that since the identification topology is stronger than the relative topology, $i_{E^{u}, E}$ and $i_{\Phi^{u}, G}$ are continuous.

Definition 7.2. A quadruple $\left(\pi^{u}, \Phi^{u}, P\left\lceil_{E^{u}}, u\right)\right.$ is called the strong holonomy reduction of $(\pi, G, P, u)$ if $\left(\pi^{u}, \Phi^{u}, P\left\lceil_{E^{u}}, u\right) \in \mathbf{C}_{0}\right.$ when $\Phi^{u}$ and $E^{u}$ are endowed with the identification topology induced by $\Phi^{u} \mid P^{u}$ and $E^{u} \mid P(\cdot, u)$ respectively, where $\mathbf{C}$ is the category introduced in Section 6 .

With the identification topology on $E^{u}$, we have the following theorem.

Theorem 7.1. Let $(\pi, G, P, u)$ (resp. $\left.\left(\pi^{\prime}, G^{\prime}, P^{\prime}, u^{\prime}\right)\right) \in \mathbf{C}_{0}$, where $\pi: E \rightarrow X$ (resp. $\left.\pi^{\prime}: E^{\prime} \rightarrow X\right)$ is a principal $G$ (resp. $\left.G^{\prime}\right)$-bundle and $P \in \mathscr{P} \mathscr{D}(\pi, \subseteq)_{G}$ (resp. $\left.P^{\prime} \in \mathscr{P} \mathscr{D}\left(\pi^{\prime}, \mathfrak{\Xi}^{\prime}\right)_{G^{\prime}}\right)$ is a parallel displacement. Suppose that $X$ is $\Xi$-connected and $\left(\pi^{u}, \Phi^{u}, P \Gamma_{E^{u}}, u\right)$ is the strong holonomy reduction of $(\pi, G, P, u)$. Let $f: X \rightarrow X^{\prime}$ be a continuous map preserving $\subseteq$ and $\mathfrak{S}^{\prime}$, and satisfying $f(\pi(u))=\pi^{\prime}\left(u^{\prime}\right)$. If $f^{\sqcup}\left(\operatorname{Ker} P^{u}\right) \subset \operatorname{Ker} P^{\prime u^{\prime}}$, then there exists a unique morphism $\left(h^{u^{\prime} u}, f, \rho^{u^{\prime} u}\right)$ : $\left(\pi^{u}, \Phi^{u}, P\left\lceil_{E^{u}}, u\right) \rightarrow\left(\pi^{\prime}, G^{\prime}, P^{\prime}, u^{\prime}\right) \quad\right.$ in $\quad$ C. In particular, $h^{u^{\prime} u}\left(E^{u}\right) \subset E^{\prime u^{\prime}}$ and $\rho^{u^{\prime} u}\left(\Phi^{u}\right) \subset \Phi^{u^{\prime}}$.

Proof. From Proposition 6.6, there exists a unique map $k^{u^{\prime} u}: E^{u} \rightarrow E^{\prime u^{\prime}}$ such that $\pi^{\prime u^{\prime}} \circ k^{u^{\prime} u}=f \circ \pi^{u}, k^{u^{\prime} u} \circ P\left\lceil_{E^{u}}=P^{\prime}\left\lceil_{E^{u^{\prime}}} \circ\left(f^{\sqcup} \times k^{u^{\prime} u}\right)\right.\right.$, and $k^{u^{\prime} u}(u)=u^{\prime}$. Since $k^{u^{\prime} u} \circ{ }_{E^{u}}\left|P(\cdot, u)={ }_{E^{\prime u^{\prime}}}\right| P^{\prime}\left(\cdot, u^{\prime}\right) \circ f^{\sqcup}$ and ${ }_{E^{u}} \mid P(\cdot, u)$ is an identification, $k^{u^{\prime} u}$ is 
continuous. Put $h^{u^{\prime} u}:=i_{E^{\prime u^{\prime}}, E^{\prime}} \circ k^{u^{\prime} u}$. Then $h^{u^{\prime} u}$ is continuous. A continuous group homomorphism $\rho^{u^{\prime} u}: \Phi^{u} \rightarrow G^{\prime}$ is defined by $\rho^{u^{\prime} u}:=T^{\prime}\left(u^{\prime}, \cdot\right) \circ h^{u^{\prime} u} \circ l_{u}$. We can see that $\left(h^{u^{\prime} u}, f, \rho^{u^{\prime} u}\right):\left(\pi^{u}, \Phi^{u}, P\left\lceil_{E^{u}}, u\right) \rightarrow\left(\pi^{\prime}, G^{\prime}, P^{\prime}, u^{\prime}\right)\right.$ is a morphism in C.

In this context, under an additional assumption we obtain the implication (iii) $\Rightarrow$ (i) in Proposition 6.4.

COROLLARY 7.2. Under the assumption of Theorem 7.1, suppose that $\left(\pi^{\prime u^{\prime}}, \Phi^{u^{\prime}}, P^{\prime} \Gamma_{E^{\prime u^{\prime}}}, u^{\prime}\right) \in \mathbf{C}_{0}$ with respect to any topology, not necessarily the relative topology or the identification topology. If $f^{\sqcup}\left(\operatorname{Ker} P^{u}\right) \subset \operatorname{Ker} P^{\prime u^{\prime}}$, then there exists a unique bundle morphism $\left(h^{u^{\prime} u}, f\right): \pi^{u} \rightarrow \pi^{\prime u^{\prime}}$ preserving $P\left\lceil_{E^{u}}\right.$ and $P^{\prime}\left\lceil_{E^{\prime u^{\prime}}}\right.$, and satisfying $h^{u^{\prime} u}(u)=u^{\prime}$.

COROllary 7.3. Suppose that $\left(\pi^{u}, \Phi^{u}, P\left\lceil_{E^{u}}, u\right)\left(\operatorname{resp} .\left(\pi^{\prime u^{\prime}}, \Phi^{u^{\prime}}, P^{\prime}\left\lceil_{E^{\prime u^{\prime}}}, u^{\prime}\right)\right)\right.\right.$ is the strong holonomy reduction of $(\pi, G, P, u)$ (resp. $\left.\left(\pi^{\prime}, G^{\prime}, P^{\prime}, u^{\prime}\right)\right)$. If $f: X \rightarrow X^{\prime}$ is a homeomorphism and $f^{\sqcup}\left(\operatorname{Ker} P^{u}\right)=\operatorname{Ker} P^{\prime u^{\prime}}$, then $\left(\pi^{u}, \Phi^{u}, P \Gamma_{E^{u}}, u\right) \cong$ $\left(\pi^{\prime u^{\prime}}, \Phi^{u^{\prime}}, P^{\prime}\left\lceil_{E^{\prime u^{\prime}}}, u^{\prime}\right)\right.$.

The following proposition is a version of Proposition 6.2 and the proof is similar to that of Proposition 6.2.

Proposition 7.4. Suppose that $\Phi^{u}$ is a topological group, $E^{u}$ is a free $\Phi^{u}$ space with the continuous translation function, that is, a principal $\Phi^{u}$-space, and $P\left\lceil_{E^{u}}\right.$ is continuous. Then $\left(\pi^{u}, \Phi^{u}, P\left\lceil_{E^{u}}, u\right)\right.$ is the strong holonomy reduction of $(\pi, \Phi, P, u)$ if and only if $s^{u}: X \rightarrow E^{u} / \Phi^{u}$ is continuous.

Now, we can state a strong holonomy reduction theorem as a version of Theorem 6.3 by showing a sufficient condition for the assumption of Proposition 7.4. We call a covering $\left(C_{\lambda}\right)_{\lambda \in \Lambda}$ of a topological space $X$ a compact covering if $C_{\lambda}$ is a compact subset of $X$ for any $\lambda \in \Lambda$.

THEOREM 7.5 (Strong Holonomy Reduction Theorem). Let $(\pi, G, P, u) \in \mathbf{C}_{0}$, where $\pi: E \rightarrow X$ is a principal $G$-bundle, $P \in \mathscr{P} \mathscr{D}(\pi, \mathfrak{S})_{G}$ is a parallel displacement, and $u \in E$. Suppose that $E$ and $X$ are Hausdorff spaces, $X$ is $\mathfrak{\Xi}$-connected and has the weak topology with respect to a countable compact covering, $\subseteq$ is a closed set in $X^{\sqcup}$, and $\mathfrak{\Xi}_{(1)} \in \mathscr{S}_{X^{2}}\left(\Delta_{X}\right)$. Then $\left(\pi^{u}, \Phi^{u}, P\left\lceil_{E^{u}}, u\right)\right.$ is the strong holonomy reduction of $(\pi, G, P, u)$. Moreover, $\pi^{u}$ is a locally trivial $\Phi^{u}$-bundle. 
We prepare some lemmas to prove this theorem. The first two lemmas are rather elementary, yet we supply a proof for the sake of completeness.

Lemma 7.6. Let $X$ and $Y$ be Hausdorff spaces. Suppose that $X$ (resp. Y) has the weak topology with respect to a compact covering $\left(X_{n}\right)_{n \in \mathbf{N}}\left(\right.$ resp. $\left.\left(Y_{m}\right)_{m \in \mathbf{N}}\right)$. Then, $X \times Y$ has a weak topology with respect to a compact covering $\left(X_{n} \times Y_{m}\right)_{(n, m) \in \mathbf{N}^{2}}$.

Proof. Put $X_{1}^{\prime}:=X_{1} \quad$ (resp. $Y_{1}^{\prime}:=Y_{1}$ ) and $X_{n}^{\prime}:=X_{n-1}^{\prime} \cup X_{n} \quad$ (resp. $Y_{n}^{\prime}:=$ $\left.Y_{n-1}^{\prime} \cup Y_{n}\right)$ for any $n \in \mathbf{N} \backslash\{1\}$. Then, $X, Y$ are $k$-spaces filtered by $\left(X_{n}^{\prime}\right)_{n \in \mathbf{N}}$, $\left(Y_{m}^{\prime}\right)_{m \in \mathbf{N}}$ respectively. From (6.5) in [17], $X \times Y$ is filtered by $\left(Z_{n}\right)_{n \in \mathbf{N}}$, where $Z_{n}=\bigcup_{i \in \mathrm{N}} X_{n}^{\prime} \times Y_{n+1-i}^{\prime}$. We will show that $X \times Y$ has the weak topology with respect to $\left(X_{n} \times Y_{m}\right)_{(n, m) \in \mathbf{N}^{2}}$. Let $A \subset X \times Y$. Suppose that $A \cap\left(X_{n} \times Y_{m}\right)$ is a closed set in $X_{n} \times Y_{m}$ for any $(n, m) \in \mathbf{N}^{2}$. Since $X_{n} \times Y_{m}$ is a closed set in $X \times Y, A \cap\left(X_{n} \times Y_{m}\right)$ is a closed set in $X \times Y$ for any $(n, m) \in \mathbf{N}^{2}$. Then, since we have

$$
A \cap Z_{n}=\bigcup_{i=1}^{n} A \cap\left(X_{i} \times Y_{n+1-i}\right)=\left(\bigcup_{i=1}^{n} A \cap\left(X_{i} \times Y_{n+1-i}\right)\right) \cap Z_{n}
$$

$A \cap Z_{n}$ is a closed set in $Z_{n}$ for any $n \in \mathbf{N}$. Thus, $A$ is a closed set in $X \times Y$ and $X \times Y$ has the weak topology with respect to $\left(X_{n} \times Y_{m}\right)_{(n, m) \in \mathbf{N}^{2}}$. Since $X_{n}, Y_{m}$ are compact sets of $X, Y$ respectively for $(n, m) \in \mathbf{N}^{2},\left(X_{n} \times Y_{m}\right)_{(n, m) \in \mathbf{N}^{2}}$ is a compact covering of $X \times Y$.

Lemma 7.7. Let $X$ and $X^{\prime}$ be Hausdorff spaces. Suppose that $X$ (resp. $X^{\prime}$ ) has a weak topology with respect to a compact covering $\left(X_{n}\right)_{n \in \mathbf{N}}\left(\right.$ resp. $\left.\left(X_{m}^{\prime}\right)_{m \in \mathbf{N}}\right)$. Then, for any Hausdorff spaces $Y, Y^{\prime}$ and identifications $f: X \rightarrow Y, f^{\prime}: X^{\prime} \rightarrow Y$, the product $f \times f^{\prime}: X \times X^{\prime} \rightarrow Y \times Y^{\prime}$ is an identification.

Proof. A map $\tilde{f}: \tilde{X}:=\bigcup_{n \in \mathbf{N}}\left(\{n\} \times X_{n}\right) \rightarrow \tilde{Y}:=\bigcup_{n \in \mathbf{N}}\left(\{n\} \times Y_{n}\right)$ is defined by assigning $(n, f(x))$ to $(n, x)$. Similarly, a map $\tilde{f}^{\prime}: \tilde{X}^{\prime} \rightarrow \tilde{Y}^{\prime}$ is defined. Since $X_{n} \times X_{m}^{\prime}$ is a compact set and $f\left(X_{n}\right) \times f^{\prime}\left(X_{m}\right)$ is a Hausdorff space, $\left.\left(f \times f^{\prime}\right)\right|_{X_{n} \times X_{m}^{\prime}}$ is an identification for any $(n, m) \in \mathbf{N}^{2}$. Thus, $\tilde{f} \times \tilde{f}^{\prime}: \tilde{X} \times \tilde{X}^{\prime} \rightarrow \tilde{Y} \times \tilde{Y}^{\prime}$ is an identification. We can see that $Y, Y^{\prime}$ have the weak topology with respect to compact coverings $\left(f\left(X_{n}\right)\right)_{n \in \mathbf{N}},\left(f^{\prime}\left(X_{n}^{\prime}\right)\right)_{n \in \mathbf{N}}$ respectively. Then from Lemma 7.6, $Y \times Y^{\prime}$ has the weak topology with respect to $\left(f\left(X_{n}\right) \times f^{\prime}\left(X_{m}^{\prime}\right)\right)_{(n, m) \in \mathbf{N}^{2}}$. Thus, a map $p: \tilde{Y} \times \tilde{Y}^{\prime} \rightarrow Y \times Y^{\prime}$ defined by $p\left(\left(n, y, m, y^{\prime}\right)\right):=\left(y, y^{\prime}\right)$ is an identifi- 
cation. Therefore, since $p \circ\left(\tilde{f} \times \tilde{f}^{\prime}\right)$ is an identification and $\left(f \times f^{\prime}\right) \circ q=$ $p \circ\left(\tilde{f} \times \tilde{f}^{\prime}\right), f \times f^{\prime}$ is an identification, where $q: \tilde{X} \times \tilde{X}^{\prime} \rightarrow X \times X^{\prime}$ is a map defined by $q\left(n, x, m, x^{\prime}\right):=\left(x, x^{\prime}\right)$.

Lemma 7.8. Let $X$ be a Hausdorff space and $\mathfrak{s} \in \mathscr{A} \mathscr{S}(X)$. Suppose that $X$ has the weak topology with respect to a countable compact covering, and $\subseteq$ is a closed set in $X^{\sqcup}$. Then for any $x \in X, \mathfrak{S}, \mathfrak{\Xi}_{X \times\{x\}}$, and $\mathfrak{\Xi}_{x}$ have the weak topology with respect to countable compact coverings respectively.

Proof. Let $\left(X_{n}\right)_{n \in \mathbf{N}}$ be a countable compact covering of $X$. For any $n \geq 0$, from Lemma 7.6, $X^{n+1}$ has the weak topology with respect to $\left(X_{i_{0}} \times \cdots \times X_{i_{n}}\right)_{\left(i_{0}, \ldots, i_{n}\right) \in \mathbf{N}^{n+1}}$. Then, $X^{\sqcup}=\bigcup_{n \geq 0} X^{n+1}$ has the weak topology

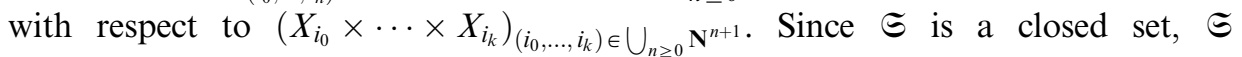
has the weak topology with respect to a countable compact covering

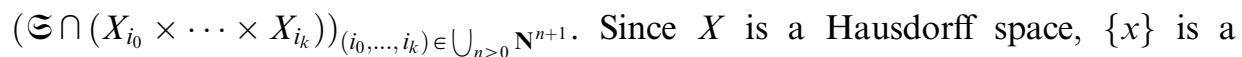
closed set in $X$ and $\mathfrak{\Xi}_{X \times\{x\}}, \mathfrak{\Xi}_{x}$ are closed sets in $\mathfrak{\Xi}$. Thus, $\mathfrak{\Xi}_{X \times\{x\}}$ (resp. $\mathfrak{\Xi}_{x}$ ) has the weak topology with respect to a countable compact covering $\left(\mathfrak{\Xi}_{X \times\{x\}} \cap\right.$



Now we are in a position to prove Theorem 7.5.

Proof of Theorem 7.5. We denote restrictions

$$
E^{u} \times_{X} E^{u}\left|\left(E^{u} \mid P(\cdot, u)\right)^{2}\right|_{\Im_{X \times\{\pi(u)\}} \times{ }_{X} \Im_{X \times\{\pi(u)\}}}
$$

and

$$
\varsigma_{X_{X} E^{u}}\left|\left(i d{ } \times E^{u} \mid P(\cdot, u)\right)\right|_{\varsigma_{X} \Xi_{X \times\{\pi(u)\}}}
$$

simply by $\left(E^{u} \mid P(\cdot, u)\right)^{2}\left\lceil_{E^{u} \times_{X} E^{u}}\right.$ and $\left(i d_{\odot} \times E_{E^{u}} \mid P(\cdot, u)\right)\left\lceil_{\varsigma_{\times_{X}} E^{u}}\right.$ respectively.

From Lemma 7.8, $\mathfrak{\subseteq}, \mathfrak{S}_{X \times\{\pi(u)\}}$, and $\mathfrak{S}_{\pi(u)}$ have the weak topology with respect to countable compact coverings respectively. Since $\Xi_{\pi(u)}$ is a closed set in $\mathfrak{\Xi}_{X \times\{\pi(u)\}}$ and the equality $\left(E^{u} \mid P(\cdot, u)\right)^{-1}\left(\left(E^{u} \mid P(\cdot, u)\right)\left(\mathfrak{\Xi}_{\pi(u)}\right)\right)=\mathfrak{\Xi}_{\pi(u)}$ holds, from Lemma 2.1, $E_{\pi(u)}^{u}|P(\cdot, u)|_{\Theta_{\pi(u)}}$ is an identification. Let $T: E^{*} \rightarrow G$ be the translation function. Since the equality $T(u, \cdot) \circ E_{\pi(u)}^{u}|P(\cdot, u)|_{\Phi_{\pi(u)}}=\Phi^{u} \mid P^{u}$ holds, $T(u, \cdot): E_{\pi(u)}^{u} \rightarrow \Phi^{u}$ is a homeomorphism. Thus, since $E_{\pi(u)}^{u}$ is a Hausdorff space, so is $\Phi^{u}$. Therefore, from Lemma 7.7, $\left(E^{u} \mid P(\cdot, u)\right)^{2}, i d_{\Im} \times E^{u} \mid P(\cdot, u)$, $E^{u}\left|P(\cdot, u) \times{ }_{\Phi^{u}}\right| P^{u}$, and $\left(\Phi^{u} \mid P^{u}\right)^{2}$ are identifications. Again, using Lemma 2.1, we 
can see that $\left(E^{u} \mid P(\cdot, u)\right)^{2}\left\lceil_{E^{u} \times X E^{u}}\right.$ and $\left(i d_{\varsigma} \times_{E^{u}} \mid P(\cdot, u)\right)\left\lceil_{\varsigma_{\times_{X}} E^{u}}\right.$ are identifications. Since

$$
\Phi^{u}|T|_{E^{u} \times_{X} E^{u}} \circ\left(E^{u} \mid P(\cdot, u)\right)^{2}\left\lceil_{E^{u} \times_{X} E^{u}}=\Phi^{u} \mid P^{u} \circ \bullet \circ\left(\cdot^{-} \times i d{\varsigma_{X \times\{\pi(u)\}}}\right)\right.
$$

and $\left(E^{u} \mid P(\cdot, u)\right)^{2}\left\lceil_{E^{u} \times X E^{u}}\right.$ is an identification, $\Phi^{u}|T|_{E^{u} \times_{X} E^{u}}$ is continuous. Let $\mu: E \times G \rightarrow E$ be the right action and $v: G \times G \rightarrow G$ the multiplication. Since

$$
\begin{gathered}
P\left\lceil_ { E ^ { u } } \circ ( i d _ { \Im } \times E _ { E ^ { u } } | P ( \cdot , u ) ) \left\lceil_{\varsigma_{X} E^{u}}={ }_{E^{u}} \mid P(\cdot, u) \circ \bullet,\right.\right. \\
E^{u}|\mu|_{E^{u} \times \Phi^{u}} \circ\left(E_{E^{u}}\left|P(\cdot, u) \times{ }_{\Phi^{u}}\right| P^{u}\right)={ }_{E^{u}} \mid P(\cdot, u) \circ \bullet,
\end{gathered}
$$

and

$$
\Phi^{u}|v|_{\Phi^{u} \times \Phi^{u}} \circ\left(\Phi^{u} \mid P^{u}\right)^{2}=\Phi^{u} \mid P^{u} \circ \bullet
$$

hold, maps $P\left\lceil_{E^{u}}, E^{u}|\mu|_{E^{u} \times \Phi^{u}}\right.$, and $\Phi_{\Phi^{u}}|v|_{\Phi^{u} \times \Phi^{u}}$ are continuous. Therefore, $\Phi^{u}$ is a topological group and $E^{u}$ is a free $\Phi^{u}$-space with the continuous translation function $\Phi^{u}|T|_{E^{u} \times{ }_{X} E^{u}}$.

By the same argument as in the proof of Theorem 6.3, $s^{u}$ is continuous. Then, from Proposition 7.4, $\left(\pi^{u}, \Phi^{u}, P\left\lceil_{E^{u}}, u\right)\right.$ is the strong holonomy reduction of $(\pi, G, P, u)$. Moreover, from Theorem 4.4 (ii), $\pi^{u}$ is locally trivial.

The following is a typical example of the base space $X$ of $\pi$ satisfying the assumption of Theorem 7.5.

EXAmPle 7.1. Let $X$ be a polyhedron of a countable simplicial complex $K$ in the weak topology. Note that $|\tau|$ is a compact set of $X$ for any $\tau \in K$. Put $U_{K}:=\bigcup_{\tau \in K}|\tau| \times|\tau|$ and $\Im_{K}:=\left\langle U_{K}\right\rangle$. As we saw in Example 4.1, $\left(\Im_{K}\right)_{(1)}=$ $U_{K} \in \mathscr{S}_{X^{2}}\left(\Delta_{X}\right)$ and $X$ is $\Im_{K}$-connected. We can see that $\Im_{K}$ is a cell subcomplex of CW-complex $X^{\sqcup}$, thus $\varsigma_{K}$ is a closed set.

\section{A Classification of Principal Bundles with Parallel Displacements}

In this section, we study a subcategory $\mathbf{C}(X, x, \Xi)$ of $\mathbf{C}$ defined in Section 6 , where $(X, x)$ is a topological space with base point $x$ and $\subseteq \in \mathscr{A} \mathscr{S}(X)$ is an admissible sequence space. Objects in $\mathbf{C}(X, x, \Xi)$ are such quadruples $(\pi, G, P, u)$, where $\pi: E \rightarrow X$ is a principal $G$-bundle, $P \in \mathscr{P} \mathscr{D}(\pi, \subseteq)_{G}$ is a parallel displacement, and $u \in E_{x}$, and morphisms in $\mathbf{C}(X, x, \Xi)$ are such homomorphisms $\left(h, i d_{X}, \rho\right):(\pi, G, P, u) \rightarrow\left(\pi^{\prime}, G^{\prime}, P^{\prime}, u^{\prime}\right)$ preserving $P$ and $P^{\prime}$, and satisfying $h(u)=u^{\prime}$. We denote by $(h, \rho)$ the morphism $\left(h, i d_{X}, \rho\right)$, and by $\mathbf{C}(X, x, \mathbb{\Xi})_{0}$ (resp. 
$\left.\mathbf{C}(X, x, \mathfrak{S})_{1}\right)$ the collection of objects (resp. morphisms) in $\mathbf{C}(X, x, \mathfrak{\Xi})$. Assuming the existence of an initial object in $\mathbf{C}(X, x, \mathfrak{S})$, we shall see that objects are classified in terms of topological group homomorphisms from the structure group of the initial object to those of bundles. Next we consider a sufficient condition for the existence of an initial object in $\mathbf{C}(X, x, \mathfrak{\Xi})$. We shall see that a specific object is an initial object if it is the strong holonomy reduction of itself. From this fact, we can see that $\left(\tilde{\pi}_{K}, \tilde{G}_{K}, \tilde{P}_{K},\left[v_{0}\right]\right)$ is an initial object in $\mathbf{C}\left(|K|, v_{0}, \Im_{K}\right)$, where $K$ is a countable connected simplicial complex in the weak topology. In particular, we obtain Theorem 5.1 in [14].

Let $\mathbf{G}$ be the category of topological groups. We denote by $\mathbf{G}_{0}$ (resp. $\mathbf{G}_{1}$ ) the collection of objects (resp. morphisms). Let $\tilde{G} \in \mathbf{G}_{0}$. An equivalence relation on $\left\{\rho \in \mathbf{G}_{1} \mid \operatorname{dom} \rho=\tilde{G}\right\}$ is defined as follows. Two morphisms $\rho, \rho^{\prime} \in \mathbf{G}_{1}$ with $\operatorname{dom} \rho=\operatorname{dom} \rho^{\prime}=\tilde{G}$ are equivalent if there exists a topological group isomorphism $\tau: \operatorname{cod} \rho \rightarrow \operatorname{cod} \rho^{\prime}$ such that $\rho^{\prime}=\tau \circ \rho$. We denote by $[\rho]$ the equivalence class of $\rho$. Then we have the following classification theorem.

THEOREM 8.1 (Classification Theorem). Let $(\tilde{\pi}, \tilde{G}, \tilde{P}, \tilde{u})$ be an initial object of $\mathbf{C}(X, x, \mathfrak{\Xi})$ with $\tilde{\pi}: \tilde{E} \rightarrow X$. Two maps

$$
\left\{\rho \in \mathbf{G}_{1} \mid \operatorname{dom} \rho=\tilde{G}\right\} \stackrel{\Lambda}{\rightarrow} \mathbf{C}(X, x, \Im)_{0} \stackrel{\Theta}{\rightarrow}\left\{\rho \in \mathbf{G}_{1} \mid \operatorname{dom} \rho=\tilde{G}\right\}
$$

are defined as follows. For $\rho \in \mathbf{G}_{1}$ with $\operatorname{dom} \rho=\tilde{G}$, put $\Lambda(\rho):=\left(\tilde{\pi}^{\rho}, \operatorname{cod} \rho\right.$, $\left.\tilde{\boldsymbol{P}}^{\rho},\left[\tilde{u}, 1_{\operatorname{cod} \rho}\right]\right)$. For $(\pi, G, P, u) \in \mathbf{C}(X, x, \Xi)_{0}$ with the unique morphism $(h, \rho)$ : $(\tilde{\pi}, \tilde{G}, \tilde{P}, \tilde{u}) \rightarrow(\pi, G, P, u)$ in $\mathbf{C}(X, x, \Xi)$, put $\Theta(\pi, G, P, u):=\rho$. Then $\Theta \circ \Lambda=i d$, and $\Lambda(\Theta(\pi, G, P, u))$ and $(\pi, G, P, u)$ are isomorphic for each $(\pi, G, P, u) \in$ $\mathbf{C}(X, x, \mathfrak{S})_{0}$. Moreover, for $\rho, \rho^{\prime} \in \mathbf{G}_{1}$ with $\operatorname{dom} \rho=\operatorname{dom} \rho^{\prime}=\tilde{G}, \rho$ and $\rho^{\prime}$ are equivalent if and only if $\Lambda(\rho)$ and $\Lambda\left(\rho^{\prime}\right)$ are isomorphic. Thus, the induced map

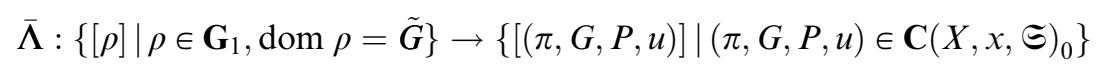

is bijective, where $[(\pi, G, P, u)]$ is the isomorphic class of $(\pi, G, P, u)$.

Proof. For $(\rho: \tilde{G} \rightarrow G) \in \mathbf{G}_{1}$, let $\left(\theta^{\rho}, \rho\right):(\tilde{\pi}, \tilde{G}) \rightarrow\left(\tilde{\pi}^{\rho}, G\right)$ be the homomorphism given by $\theta^{\rho}(v):=\left[v, 1_{G}\right]$ for $v \in \tilde{E}$ as in Section 2. From Proposition 4.7, $\left(\theta^{\rho}, \rho\right)$ preserves $\tilde{\boldsymbol{P}}$ and $\tilde{\boldsymbol{P}}^{\rho}$. Thus $\left(\theta^{\rho}, \rho\right):(\tilde{\pi}, \tilde{\boldsymbol{G}}, \tilde{\boldsymbol{P}}, \tilde{u}) \rightarrow\left(\tilde{\pi}^{\rho}, G, \tilde{\boldsymbol{P}}^{\rho},\left[\tilde{u}, 1_{G}\right]\right)$ is a morphism in $\mathbf{C}(X, x, \mathfrak{S})$. Since $(\tilde{\pi}, \tilde{G}, \tilde{P}, \tilde{u})$ is an initial object, $\left(\theta^{\rho}, \rho\right)$ is the unique morphism from $(\tilde{\pi}, \tilde{G}, \tilde{P}, \tilde{u})$ to $\left(\tilde{\pi}^{\rho}, G, \tilde{P}^{\rho},\left[\tilde{u}, 1_{G}\right]\right)$. Thus $\Theta(\Lambda(\rho))=$ $\Theta\left(\tilde{\pi}^{\rho}, G, \tilde{P}^{\rho},\left[\tilde{u}, 1_{G}\right]\right)=\rho$.

Let $(\pi, G, P, u) \in \mathbf{C}(X, x, \widetilde{\Xi})_{0}$ and $(h, \rho):(\tilde{\pi}, \tilde{G}, \tilde{P}, \tilde{u}) \rightarrow(\pi, G, P, u)$ be the unique morphism in $\mathbf{C}(X, x, \mathfrak{S})$. Let $\left(h^{\rho}, i d_{G}\right):\left(\tilde{\pi}^{\rho}, G\right) \rightarrow(\pi, G)$ be the $(X, G)$ - 
morphism given by $h^{\rho}([v, a]):=h(v) a$ for $[v, a] \in \tilde{E}^{\rho}$ as in Section 2. From Proposition 4.8, $\left(h^{\rho}, i d_{G}\right)$ preserves $\tilde{P}^{\rho}$ and $P$. Since $h^{\rho} \circ \theta^{\rho}=h$, we have $h^{\rho}\left(\left[\tilde{u}, 1_{G}\right]\right)=h(\tilde{u})=u$. From Corollary 2.4, $\left(h^{\rho}, i d_{G}\right)$ is an $(X, G)$-isomorphism. Thus $\left(h^{\rho}, i d_{G}\right): \Lambda(\Theta(\pi, G, P, u)) \rightarrow(\pi, G, P, u)$ is an isomorphism in $\mathbf{C}(X, x, \Xi)$.

Suppose that $[\rho: \tilde{G} \rightarrow G]=\left[\rho^{\prime}: \tilde{G} \rightarrow G^{\prime}\right]$ and let $\tau: G \rightarrow G^{\prime}$ be a topological group isomorphism such that $\rho^{\prime}=\tau \circ \rho$. A map $k: \tilde{E}^{\rho} \rightarrow \tilde{E}^{\rho^{\prime}}$ is defined by $k([v, a]):=[v, \tau(a)]^{\prime}$ for $[v, a] \in \tilde{E}^{\rho}$. The equalities

$$
\left[v b, \tau\left(\rho(b)^{-1} a\right)\right]^{\prime}=\left[v b, \tau(\rho(b))^{-1} \tau(a)\right]^{\prime}=\left[v b, \rho^{\prime}(b)^{-1} \tau(a)\right]^{\prime}=[v, \tau(a)]^{\prime}
$$

imply that $k$ is well-defined. Since $k \circ q_{\tilde{G}}^{\tilde{E} \times G}=q_{\tilde{G}}^{\tilde{E} \times G^{\prime}} \circ\left(i d_{\tilde{E}} \times \tau\right)$ and $q_{\tilde{G}}^{\tilde{E} \times G}$ is an identification, $k$ is continuous. From the equalities

$\tilde{\pi}^{\rho^{\prime}} \circ k \circ q_{\tilde{G}}^{\tilde{E} \times G}=\tilde{\pi}^{\rho^{\prime}} \circ q_{\tilde{G}}^{\tilde{E} \times G^{\prime}} \circ\left(i d_{\tilde{E}} \times \tau\right)=\tilde{\pi} \circ \operatorname{pr}_{1} \circ\left(i d_{\tilde{E}} \times \tau\right)=\tilde{\pi} \circ \operatorname{pr}_{1}=\tilde{\pi}^{\rho} \circ q_{\tilde{G}}^{\tilde{E} \times G}$,

we have $\tilde{\pi}^{\rho^{\prime}} \circ k=\tilde{\pi}^{\rho}$, hence, $k: \tilde{\pi}^{\rho} \rightarrow \tilde{\pi}^{\rho^{\prime}}$ is a bundle morphism. Since

$$
k([v, a] c)=k([v, a c])=[v, \tau(a c)]^{\prime}=[v, \tau(a)]^{\prime} \tau(c)=k([v, a]) \tau(c)
$$

for $([v, a], c) \in \tilde{E}^{\rho} \times G,(k, \tau):\left(\tilde{\pi}^{\rho}, G\right) \rightarrow\left(\tilde{\pi}^{\rho^{\prime}}, G^{\prime}\right)$ is a homomorphism. Since the equalities

$$
\begin{aligned}
k\left(\tilde{\boldsymbol{P}}^{\rho}(\mathbf{x},[v, a])\right) & =k([\tilde{\boldsymbol{P}}(\mathbf{x}, v), a])=[\tilde{\boldsymbol{P}}(\mathbf{x}, v), \tau(a)]^{\prime} \\
& =\tilde{\boldsymbol{P}}^{\rho^{\prime}}\left(\mathbf{x},[v, \tau(a)]^{\prime}\right)=\tilde{\boldsymbol{P}}^{\rho^{\prime}}(\mathbf{x}, k([v, a]))
\end{aligned}
$$

hold for $(\mathbf{x},[v, a]) \in\left(\left.p_{0}\right|_{\Im}\right)^{*} \tilde{E}^{\rho}, \quad(k, \tau)$ preserves $\tilde{P}^{\rho}$ and $\tilde{P}^{\rho^{\prime}}$. The equality $k\left(\left[\tilde{u}, 1_{G}\right]\right)=\left[\tilde{u}, 1_{G^{\prime}}\right]^{\prime}$ implies that $(k, \tau):\left(\tilde{\pi}^{\rho}, G, \tilde{P}^{\rho},\left[\tilde{u}, 1_{G}\right]\right) \rightarrow\left(\tilde{\pi}^{\rho^{\prime}}, G^{\prime}, \tilde{P}^{\rho^{\prime}},\left[\tilde{u}, 1_{G^{\prime}}\right]^{\prime}\right)$ is a morphism. By the same argument, a bundle morphism $k^{\prime}: \tilde{\pi}^{\rho^{\prime}} \rightarrow \tilde{\pi}^{\rho}$ is defined by $k^{\prime}\left([v, a]^{\prime}\right):=\left[v, \tau^{-1}(a)\right]$ for $[v, a]^{\prime} \in \tilde{E}^{\rho^{\prime}}$ and $\left(k^{\prime}, \tau^{-1}\right):\left(\tilde{\pi}^{\rho^{\prime}}, G^{\prime}, \tilde{P}^{\rho^{\prime}}\right.$, $\left.\left[\tilde{u}, 1_{G^{\prime}}\right]^{\prime}\right) \rightarrow\left(\tilde{\pi}^{\rho}, G, \tilde{P}^{\rho},\left[\tilde{u}, 1_{G}\right]\right)$ is a morphism. We can see that $\left(k^{\prime}, \tau^{-1}\right)=(k, \tau)^{-1}$. Thus $\left(\tilde{\pi}^{\rho}, G, \tilde{P}^{\rho},\left[\tilde{u}, 1_{G}\right]\right)$ and $\left(\tilde{\pi}^{\rho^{\prime}}, G^{\prime}, \tilde{P}^{\rho^{\prime}},\left[\tilde{u}, 1_{G^{\prime}}\right]^{\prime}\right)$ are isomorphic.

Conversely, suppose that $\left(\tilde{\pi}^{\rho}, G, \tilde{P}^{\rho},\left[\tilde{u}, 1_{G}\right]\right)$ and $\left(\tilde{\pi}^{\rho^{\prime}}, G^{\prime}, \tilde{P}^{\rho^{\prime}},\left[\tilde{u}, 1_{G^{\prime}}\right]^{\prime}\right)$ are isomorphic, and let $(k, \tau):\left(\tilde{\pi}^{\rho}, G, \tilde{P}^{\rho},\left[\tilde{u}, 1_{G}\right]\right) \rightarrow\left(\tilde{\pi}^{\rho^{\prime}}, G^{\prime}, \tilde{P}^{\rho^{\prime}},\left[\tilde{u}, 1_{G^{\prime}}\right]^{\prime}\right)$ be an isomorphism. Then $\left(k \circ \theta^{\rho}, \tau \circ \rho\right):(\tilde{\pi}, \tilde{G}, \tilde{P}, \tilde{u}) \rightarrow\left(\tilde{\pi}^{\rho^{\prime}}, G^{\prime}, \tilde{P}^{\rho^{\prime}},\left[\tilde{u}, 1_{G^{\prime}}\right]^{\prime}\right)$ is a morphism. Since $(\tilde{\pi}, \tilde{\boldsymbol{G}}, \tilde{P}, \tilde{u})$ is an initial object, uniqueness of the morphism implies $\left(k \circ \theta^{\rho}, \tau \circ \rho\right)=\left(\theta^{\rho^{\prime}}, \rho^{\prime}\right)$. Thus $[\rho]=\left[\rho^{\prime}\right]$.

In the smooth category, flat principal $G$-bundles over a fixed base space are classified by group homomorphisms from the fundamental group of the base space to $G$. We can think of Theorem 8.1 as a topological counterpart of the 
classification theorem for flat bundles in the smooth category. Note that our theorem does not require any kind of flatness of the parallel displacement.

Next we consider a relation between an initial object and its holonomy reduction.

Proposition 8.2. Let $(\pi, G, P, u) \in \mathbf{C}(X, x, \Xi)_{0}$ with $\pi: E \rightarrow X$ and $\left(\pi^{u}, \Phi^{u}\right.$, $\left.P \Gamma_{E^{u}}, u\right)$ be the holonomy reduction of $(\pi, G, P, u)$. If $(\pi, G, P, u)$ is an initial object, then $\left(\pi^{u}, \Phi^{u}, P \Gamma_{E^{u}}, u\right)=(\pi, G, P, u)$.

Proof. Since $(\pi, G, P, u)$ is an initial object, there exists a unique morphism $(h, \rho):(\pi, G, P, u) \rightarrow\left(\pi^{u}, \Phi^{u}, P\left\lceil_{E^{u}}, u\right)\right.$ in $\mathbf{C}(X, x, \mathbb{S})$. Moreover, since $\left(\pi^{u}, \Phi^{u}\right.$, $P\left\lceil_{E^{u}}, u\right)$ is a holonomy reduction of $(\pi, G, P, u),\left(i_{E^{u}, E}, i_{\Phi^{u}, G}\right):\left(\pi^{u}, \Phi^{u}, P\left\lceil_{E^{u}}, u\right) \rightarrow\right.$ $(\pi, G, P, u)$ is a morphism in $\mathbf{C}(X, x, \mathfrak{\Xi})$. Thus

$$
\left(i_{E^{u}, E} \circ h, i_{\Phi^{u}, G} \circ \rho\right)=\left(i_{E^{u}, E}, i_{\Phi^{u}, G}\right) \circ(h, \rho):(\pi, G, P, u) \rightarrow(\pi, G, P, u)
$$

is a morphism in $\mathbf{C}(X, x, \mathfrak{\Xi})$. Since $\left(i d_{E}, i d_{G}\right):(\pi, G, P, u) \rightarrow(\pi, G, P, u)$ is also a morphism in $\mathbf{C}(X, x, \mathfrak{S})$, from the uniqueness of the morphism, we have $\left(i_{E^{u}, E} \circ h, i_{\Phi^{u}, G} \circ \rho\right)=\left(i d_{E}, i d_{G}\right)$. Thus $i_{E^{u}, E}$ and $i_{\Phi^{u}, G}$ are surjective, that is, $E^{u}=E$ and $\Phi^{u}=G$.

The converse of this proposition does not hold in general. Namely, even if $(\pi, G, P, u)$ is the holonomy reduction of itself, it is not necessarily an initial object. We shall see that if an object is the strong holonomy reduction of itself, then it is an initial object.

Let $(X, x)$ be a topological space with base point $x, U \subset X^{2}$ a symmetric subspace such that $\Delta_{X} \subset U$, and $\mathfrak{S}:=\langle U\rangle$. An equivalence relation on $\mathfrak{S}$ is generated by the relations

$$
\left(x_{n}, \ldots, x_{i}, \ldots, x_{0}\right) \sim\left(x_{n}, \ldots, \hat{x}_{i}, \ldots, x_{0}\right)
$$

whenever either $x_{i}=x_{i-1}$ or $x_{i+1}=x_{i-1}$, where the symbol $\hat{x}$ denotes deletion of $x$. We denote by $q: \mathfrak{S} \rightarrow \mathfrak{S} / \sim$ the natural projection, and by $\left[x_{n} \ldots, x_{0}\right]$ the equivalence class of $\left(x_{n}, \ldots, x_{0}\right)$. Put

$$
\begin{aligned}
& \tilde{\Xi}_{U}:=\Xi / \sim, \\
& \tilde{E}_{U}:=\left\{\left[x_{n}, \ldots, x_{1}, x_{0}\right] \in \tilde{\Xi}_{U} \mid x_{0}=x\right\}=q\left(\left(p_{0} \mid \Subset\right)^{-1}(\{x\})\right), \\
& \tilde{G}_{U}:=\left\{\left[x_{n}, \ldots, x_{1}, x\right] \in \tilde{E}_{U} \mid x_{n}=x\right\}=q\left(\Xi_{x}\right),
\end{aligned}
$$


where a topology of $\tilde{\mathfrak{S}}_{U}$ is the quotient topology and consider $\tilde{E}_{U}$ and $\tilde{G}_{U}$ as subspaces. A map $\tilde{\pi}_{U}: \tilde{E}_{U} \rightarrow X$ is defined by $\tilde{\pi}_{U}([\mathbf{x}]):=p_{\infty}(\mathbf{x})$. The subset $\tilde{G}_{U}$ is a group with a binary operation $\tilde{v}_{U}$ given by $\tilde{v}_{U}([\mathbf{x}],[\mathbf{y}]):=[\mathbf{x} \bullet \mathbf{y}]$, a unary operation.$^{-1}$ given by $[\mathbf{x}]^{-1}:=\left[\mathbf{x}^{-}\right]$, and an identity element $[x]$. A right action $\tilde{\mu}_{U}: \tilde{E}_{U} \times \tilde{G}_{U} \rightarrow \tilde{E}_{U}$ is defined by $[\mathbf{x}][\mathbf{y}]:=[\mathbf{x} \bullet \mathbf{y}]$. If $[\mathbf{x}][\mathbf{y}]=[\mathbf{x}]$ for $([\mathbf{x}],[\mathbf{y}]) \in$ $\tilde{E}_{U} \times \tilde{G}_{U}$, then $[\mathbf{y}]=\left[\mathbf{x}^{-}\right][\mathbf{x}][\mathbf{y}]=\left[\mathbf{x}^{-}\right][\mathbf{x}]=[x]$. Thus the action $\tilde{\mu}_{U}$ is free. Since $\tilde{G}_{U}$ is the subspace of $\tilde{E}_{U}, l_{[x]}=i d_{\tilde{G}_{U}}: \tilde{G}_{U} \rightarrow\left(\tilde{E}_{U}\right)_{x}=\tilde{G}_{U}$ is a homeomorphism. A translation function $\tilde{T}_{U}: \tilde{E}_{U} \times_{X} \tilde{E}_{U} \rightarrow \tilde{G}_{U}$ is defined by $\tilde{T}_{U}([\mathbf{x}],[\mathbf{y}]):=\left[\mathbf{x}^{-} \bullet \mathbf{y}\right]$. A map $\tilde{P}_{U}: \mathfrak{S} \times_{X} \tilde{E}_{U} \rightarrow \tilde{E}_{U}$ is defined by $\tilde{P}_{U}(\mathbf{x},[\mathbf{y}]):=[\mathbf{x} \bullet \mathbf{y}]$. For any $(\mathbf{x},[\mathbf{y}]) \in$ $\Subset \times{ }_{X} \tilde{E}_{U}$,

$$
\tilde{\boldsymbol{P}}_{U}\left(\mathbf{x}^{-}, \tilde{\boldsymbol{P}}_{U}(\mathbf{x},[\mathbf{y}])\right)=\left[\mathbf{x}^{-} \bullet \mathbf{x} \bullet \mathbf{y}\right]=[\mathbf{y}]
$$

Similarly, we have $\left(\tilde{\boldsymbol{P}}_{U}\right)_{\mathbf{x}} \circ\left(\tilde{\boldsymbol{P}}_{U}\right)_{\mathbf{x}^{-}}=i d_{\left(\tilde{E}_{U}\right)_{p \infty}(\mathbf{x})}$, and consequently $\left(\tilde{\boldsymbol{P}}_{U}\right)_{\mathbf{x}^{-}}=$ $\left(\left(\tilde{\boldsymbol{P}}_{U}\right)_{\mathbf{x}}\right)^{-1}$. For $((\mathbf{x},[\mathbf{y}]),[\mathbf{z}]) \in\left(\boldsymbol{S} \times_{X} \tilde{E}_{U}\right) \times \tilde{G}_{U}$, we have

$$
\left.\tilde{P}_{U}(\mathbf{x},[\mathbf{y}][\mathbf{z}])\right)=[\mathbf{x} \bullet \mathbf{y} \bullet \mathbf{z}]=[\mathbf{x} \bullet \mathbf{y}][\mathbf{z}]=\tilde{P}_{U}(\mathbf{x},[\mathbf{y}])[\mathbf{z}] .
$$

A map $\tilde{\pi}_{U} / \tilde{G}_{U}: \tilde{E}_{U} / \tilde{G}_{U} \rightarrow X$ is defined by $\tilde{\pi}_{U} / \tilde{G}_{U}([[\mathbf{x}]]):=\tilde{\pi}_{U}([\mathbf{x}])$ (see Section 2). Since for $([\mathbf{x}],[\mathbf{y}]) \in \tilde{E}_{U} \times \tilde{G}_{U}$,

$$
\tilde{\pi}_{U}([\mathbf{x}][\mathbf{y}])=p_{\infty}(\mathbf{x} \bullet \mathbf{y})=p_{\infty}(\mathbf{x})=\tilde{\pi}_{U}([\mathbf{x}]),
$$

$\tilde{\pi}_{U} / \tilde{G}_{U}$ is well-defined. Suppose further that $X$ is $\Xi$-connected. A map $\tilde{s}_{U}: X$ $\rightarrow \tilde{E}_{U} / \tilde{G}_{U}$ is defined as follows. Let $y \in X$ and $\mathbf{x} \in \Theta_{y, x}$. Then put $\tilde{s}_{U}(y):=$ $\left[\tilde{P}_{U}(\mathbf{x},[x])\right]=[[\mathbf{x}]]$. We can see $\tilde{s}_{U}=\left(\tilde{\pi}_{U} / \tilde{G}_{U}\right)^{-1}$. For simplicity, we denote by $q\left\lceil_{\tilde{E}_{U}}\right.$ (resp. $q\left\lceil_{\tilde{G}_{U}}\right.$ ) the restriction $\tilde{E}_{U}|q|_{\varsigma_{X \times\{x\}}}$ (resp. $\tilde{G}_{U}|q|_{\varsigma_{x}}$ ). Similarly, we denote the restrictions

$$
\tilde{E}_{U} \times{ }_{X} \tilde{E}_{U} \mid\left(\left.q\left\lceil_{\tilde{E}_{U}}\right)^{2}\right|_{\Xi_{X \times\{x\}} \times{ }_{X} \Xi_{X \times\{x\}}}\right.
$$

and

$$
\Xi_{\times_{X} \tilde{E}_{U}} \mid\left(i d_{\Xi} \times\left. q\left\lceil_{\tilde{E}_{U}}\right)\right|_{\varsigma_{\times} \Xi_{X \times\{x\}}}\right.
$$

simply by $\left(q\left\lceil_{\tilde{E}_{U}}\right)^{2}\left\lceil_{\tilde{E}_{U} \times_{X} \tilde{E}_{U}}\right.\right.$ and $\left(i d_{\subseteq} \times q\left\lceil_{\tilde{E}_{U}}\right)\left\lceil_{\varsigma_{\times_{X}} \tilde{E}_{U}}\right.\right.$ respectively. We have a sufficient condition for maps $\tilde{\pi}_{U}, \tilde{v}_{U}, \tilde{\mu}_{U}, \tilde{T}_{U}$, and $\tilde{P}_{U}$ to be continuous.

Proposition 8.3. Suppose that $X$ and $\tilde{E}_{U}$ are Hausdorff spaces, $X$ is $\mathbb{\Xi}$ connected and has the weak topology with respect to a countable compact covering, and $\subseteq$ is a closed set in $X^{\sqcup}$. Then 


$$
\begin{aligned}
& q\left\lceil_{\tilde{E}_{U}}:\left(p_{0} \mid \Subset\right)^{-1}(\{x\}) \rightarrow \tilde{E}_{U},\right. \\
& q\left\lceil_{\tilde{G}_{U}}: \boldsymbol{\Xi}_{x} \rightarrow \tilde{\boldsymbol{G}}_{U},\right. \\
& \left(q \lceil _ { \tilde { E } _ { U } } ) ^ { 2 } \left\lceil_{\tilde{E}_{U} \times \tilde{E}_{U}}: \boldsymbol{\Xi}_{X \times\{x\}} \times{ }_{X} \boldsymbol{\Xi}_{X \times\{x\}} \rightarrow \tilde{E}_{U} \times_{X} \tilde{E}_{U},\right.\right.
\end{aligned}
$$

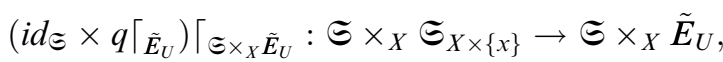

$$
\begin{aligned}
& q\left\lceil_{\tilde{E}_{U}} \times q\left\lceil_{\tilde{G}_{U}}: \mathfrak{\Xi}_{X \times\{x\}} \times \mathfrak{\Xi}_{x} \rightarrow \tilde{E}_{U} \times \tilde{G}_{U},\right.\right.
\end{aligned}
$$

and

$$
\left(q \Gamma_{\tilde{G}_{U}}\right)^{2}: \boldsymbol{\Xi}_{x} \times \boldsymbol{\Xi}_{x} \rightarrow \tilde{\boldsymbol{G}}_{U} \times \tilde{\boldsymbol{G}}_{U}
$$

are identifications. In particular, $\tilde{G}_{U}$ is a topological group, and $\tilde{\pi}_{U}, \tilde{\mu}_{U}, \tilde{T}_{U}$, and $\tilde{P}_{U}$ are continuous.

Proof. Since $\mathfrak{\Xi}_{X \times\{x\}}$ is closed in $\mathfrak{\subseteq}$ and $q^{-1}\left(q\left(\mathfrak{\Xi}_{X \times\{x\}}\right)\right)=\mathfrak{\Xi}_{X \times\{x\}}$, from Lemma 2.1, $q \Gamma_{\tilde{E}_{U}}$ is an identification. Similarly, $q \Gamma_{\tilde{G}_{U}}$ is an identification. From Lemmas 7.7 and 7.8, $\left(q \Gamma_{\tilde{E}_{U}}\right)^{2}, i d_{\subseteq} \times q \Gamma_{\tilde{E}_{U}}, q \Gamma_{\tilde{E}_{U}} \times q \Gamma_{\tilde{G}_{U}}$, and $\left(q \Gamma_{\tilde{G}_{U}}\right)^{2}$ are identifications. Again, from Lemma 2.1, we can see that $\left(q \Gamma_{\tilde{E}_{U}}\right)^{2} \Gamma_{\tilde{E}_{U} \times X \tilde{E}_{U}}$ and $\left(i d_{\Im} \times q\left\lceil_{\tilde{E}_{U}}\right)\left\lceil_{\varsigma_{\times} \tilde{E}_{U}}\right.\right.$ are identifications. Since $\tilde{\pi}_{U} \circ q\left\lceil_{\tilde{E}_{U}}=\left.p_{\infty}\right|_{\varsigma_{X \times\{x\}}}, \tilde{\pi}_{U}\right.$ is continuous. Similarly, we can see that $\cdot^{-1}, \tilde{\mu}_{U}, \tilde{T}_{U}$, and $\tilde{P}_{U}$ are continuous.

By a straightforward argument, we have the following lemma.

Lemma 8.4. Let $(\pi, G, P, u) \in \mathbf{C}(X, x, \mathfrak{\Xi})_{0}$. Then, the following conditions are equivalent: (i) $\pi^{u}=\pi$, (ii) $\Phi^{u}=G$, and (iii) $P\left\lceil_{E^{u}}=P\right.$.

Maps $\tilde{s}_{U}, \tilde{\pi}_{U}, \tilde{\mu}_{U}, \tilde{T}_{U}$, and $\tilde{P}_{U}$ and the quadruple $\left(\tilde{\pi}_{U}, \tilde{G}_{U}, \tilde{P}_{U},[x]\right)$ have the following properties.

Proposition 8.5. (i) If $U \in \mathscr{S}_{X^{2}}\left(\Delta_{X}\right)$ (Definition 4.2), then $\tilde{s}_{U}$ is continuous.

(ii) Suppose that $\tilde{G}_{U}$ is a topological group and $\tilde{\pi}_{U}, \tilde{\mu}_{U}, \tilde{T}_{U}$, and $\tilde{P}_{U}$ are continuous. Then $\left(\tilde{\pi}_{U}, \tilde{G}_{U}, \tilde{P}_{U},[x]\right) \in \mathbf{C}(X, x, \boldsymbol{\Xi})_{0}$ if and only if $\tilde{s}_{U}$ is continuous. In this case, if $U \in \mathscr{S}_{X^{2}}\left(\Delta_{X}\right)$, then $\tilde{\pi}_{U}$ is a locally trivial $\tilde{G}_{U}$-bundle.

(iii) Suppose that $\left(\tilde{\pi}_{U}, \tilde{G}_{U}, \tilde{P}_{U},[x]\right) \in \mathbf{C}(X, x, \mathfrak{\Xi})_{0}$. If $q\left\lceil_{\tilde{E}_{U}}\right.$ and $q\left\lceil_{\tilde{G}_{U}}\right.$ are identifications, then $\left(\tilde{\pi}_{U}, \tilde{G}_{U}, \tilde{P}_{U},[x]\right)$ is the strong holonomy reduction of itself. 
Proof. Firstly, we show (i) and (ii). If $U \in \mathscr{S}_{X^{2}}\left(\Delta_{X}\right)$, from Proposition 4.2, $\left.p_{\infty}\right|_{\varsigma_{X \times\{x\}}}$ is an identification. Then, since $\left.\tilde{s}_{U} \circ p_{\infty}\right|_{\varsigma_{X \times\{x\}}}=q_{\tilde{G}_{U}}^{\tilde{E}_{U}} \circ q \Gamma_{\tilde{E}_{U}}, s^{u}$ is continuous, and we obtain (i). Under the assumption that $\tilde{\pi}_{U}$ is continuous, $\tilde{s}_{U}$ is continuous if and only if $\tilde{\pi}_{U} / \tilde{G}_{U}$ is a homeomorphism. Then, from Lemma 2.3, we obtain (ii).

Secondly, we show (iii). Note that $\tilde{E}_{U} \mid \tilde{P}_{U}(\cdot,[x])=q\left\lceil_{\tilde{E}_{U}}\right.$ and ${ }_{\Phi^{[x]}} \mid\left(\tilde{P}_{U}\right)^{[x]}=$ $\Phi^{[x]} \mid\left(q\left\lceil_{\tilde{G}_{U}}\right)\right.$. By the definition, if $\left(\left(\tilde{\pi}_{U}\right)^{[x]}, \Phi^{[x]}, \tilde{P}_{U}\left\lceil_{\left(\tilde{E}_{U}\right)^{[x]}},[x]\right) \in \mathbf{C}(X, x, \boldsymbol{S})_{0}\right.$, it is the strong holonomy reduction of $\left(\tilde{\pi}_{U}, \tilde{G}_{U}, \tilde{P}_{U},[x]\right)$. For $[\mathrm{x}] \in \tilde{G}_{U}$, since $[\mathrm{x}]=$ $\tilde{T}_{U}\left([x], \tilde{P}_{U}([\mathrm{x}],[x]) \in \Phi^{[x]}\right.$, we have $\tilde{G}_{U}=\Phi^{[x]}$. From Lemma 8.4, $\left(\left(\tilde{\pi}_{U}\right)^{[x]}, \Phi^{[x]}\right.$, $\tilde{P}_{U}\left\lceil_{\left(\tilde{E}_{U}\right)^{[x]}},[x]\right)=\left(\tilde{\pi}_{U}, \tilde{G}_{U}, \tilde{P}_{U},[x]\right)$ and this complete the proof.

In particular, from the viewpoint of a holonomy reduction, we obtain the following proposition.

Proposition 8.6 (cf. [14]). Let $X$ be a polyhedron of a countable connected simplicial complex $K$ in the weak topology and $\tilde{\pi}_{K}$ the universal bundle. Then, $\left(\tilde{\pi}_{K}, \tilde{G}_{K}, \tilde{P}_{K},\left[v_{0}\right]\right)$ is the strong holonomy reduction of itself.

Proof. Put $U_{K}:=\bigcup_{\tau \in K}|\tau| \times|\tau|$, and $\Im_{K}:=\left\langle U_{K}\right\rangle$. Note that $X$ is a Hausdorff space. As we have already seen in Example 7.1, $X$ has the weak topology with respect to a countable compact covering $\{|\tau| \mid \tau \in K\}, \quad X$ is $\Im_{K}$-connected, $U_{K} \in \mathscr{S}_{X^{2}}\left(\Delta_{X}\right)$, and $\Im_{K}$ is a closed set in $X^{\sqcup}$. From Lemma 3.2 in [14], $\tilde{E}_{U_{K}}=\tilde{E}_{K}$ is a Hausdorff space. Thus, from Proposition 8.3, $\tilde{G}_{U_{K}}=\tilde{G}_{K}$ is a topological group, $\tilde{\pi}_{U_{K}}=\tilde{\pi}_{K}$ is a principal $\tilde{G}_{K}$-bundle, and $\tilde{P}_{K} \in$ $\mathscr{P} \mathscr{D}\left(\tilde{\pi}_{K}, \Xi_{K}\right)_{\tilde{G}_{K}}$. Moreover, from (i) in Proposition 8.5, $\tilde{s}_{U_{K}}$ is continuous. Then, from (ii) in Proposition 8.5, $\left(\tilde{\pi}_{K}, \tilde{G}_{K}, \tilde{P}_{K},\left[v_{0}\right]\right) \in \mathbf{C}\left(X, x, \Xi_{K}\right)_{0}$. By using Lemma 2.1, we can see that $q \Gamma_{\tilde{E}_{K}}$ and $q\left\lceil_{\tilde{G}_{K}}\right.$ are identifications. Thus, from (iii) in Proposition $8.5,\left(\tilde{\pi}_{K}, \tilde{G}_{K}, \tilde{P}_{K},\left[v_{0}\right]\right)$ is the strong holonomy reduction of itself.

As we have already mentioned, an object which is the holonomy reduction of itself is not necessarily an initial object. While, if the quadruple $\left(\tilde{\pi}_{U}, \tilde{G}_{U}, \tilde{P}_{U},[x]\right)$ defined above is the strong holonomy reduction of itself, then it is an initial object.

THEOREM 8.7. If the quadruple $\left(\tilde{\pi}_{U}, \tilde{G}_{U}, \tilde{P}_{U},[x]\right)$ is the strong holonomy reduction of itself, then it is an initial object in $\mathbf{C}(X, x, \mathbb{S})$. 



Then,

$$
\left[x, x_{n-1}, \ldots, x_{1}, x\right]=\left(\tilde{P}_{U}\right)^{[x]}\left(x, x_{n-1}, \ldots, x_{1}, x\right)=[x],
$$

that is, $\left(x, x_{n-1}, \ldots, x_{1}, x\right) \sim x$. By the definition of $\sim$, there exists $m \in \mathbf{N} \cup\{0\}$ and a sequence $\left(x, x_{n-1}, \ldots, x_{1}, x\right)=\mathbf{x}_{0}, \mathbf{x}_{1}, \ldots, \mathbf{x}_{m}=x$ such that for any $j \in$ $\{1, \ldots, m\}$ at least one of the following conditions hold:

(1) there exist $k \in \mathbf{N}$ and $i \in\{1, \ldots, k\}$ such that

$$
\mathbf{x}_{j-1}=\left(y_{k}, \ldots, y_{1}, y_{0}\right), \quad \mathbf{x}_{j}=\left(y_{k}, \ldots, \hat{y}_{i}, \ldots, y_{1}, y_{0}\right)
$$

and $y_{i}=y_{i-1}$ or $y_{i+1}=y_{i-1}$, or

(2) there exist $k \in \mathbf{N}$ and $i \in\{1, \ldots, k\}$ such that

$$
\mathbf{x}_{j-1}=\left(y_{k}, \ldots, \hat{y}_{i}, \ldots, y_{1}, y_{0}\right), \quad \mathbf{x}_{j}=\left(y_{k}, \ldots, y_{1}, y_{0}\right)
$$

and $y_{i}=y_{i-1}$ or $y_{i+1}=y_{i-1}$.

In case (1), whether $y_{i}=y_{i-1}$ or $y_{i+1}=y_{i-1}$, we have

$$
\begin{aligned}
P\left(\mathbf{x}_{j-1}, u\right) & =P\left(\left(y_{k}, \ldots, y_{1}, y_{0}\right), u\right) \\
& =\left(P_{\left(y_{k}, y_{k-1}\right)} \circ \cdots \circ P_{\left(y_{i+1}, y_{i}\right)} \circ P_{\left(y_{i}, y_{i-1}\right)} \circ \cdots \circ P_{\left(y_{1}, y_{0}\right)}\right)(u) \\
& =\left(P_{\left(y_{k}, y_{k-1}\right)} \circ \cdots \circ P_{\left(y_{i+1}, y_{i-1}\right)} \circ \cdots \circ P_{\left(y_{1}, y_{0}\right)}\right)(u) \\
& =P\left(\left(y_{k}, \ldots, \hat{y}_{i}, \ldots, y_{1}, y_{0}\right), u\right)=P\left(\mathbf{x}_{j}, u\right) .
\end{aligned}
$$

In case (2), we get $P\left(\mathbf{x}_{j-1}, u\right)=P\left(\mathbf{x}_{j}, u\right)$ by a similar computation to that of case (1). Then, we have

$$
P\left(\mathbf{x}_{0}, u\right)=P\left(\mathbf{x}_{1}, u\right)=\cdots=P(x, u)=u .
$$

Thus $\left(x, x_{n-1}, \ldots, x_{1}, x\right) \in \operatorname{Ker} P^{u}$, that is, $\operatorname{Ker}\left(\tilde{P}_{U}\right)^{[x]} \subset \operatorname{Ker} P^{u}$. Then, from Theorem 7.1, there exists a unique morphism $\left(h^{u[x]}, \rho^{u[x]}\right):\left(\tilde{\pi}_{U}, \tilde{G}_{U}, \tilde{P}_{U},[x]\right) \rightarrow$ $(\pi, G, P, u)$.

As a corollary, we obtain Theorem 5.1 in [14].

COROLLARY 8.8 ([14, Theorem 5.1]). Let $X$ be a polyhedron of a countable connected simplicial complex $K$ in the weak topology and $\tilde{\pi}_{K}: \tilde{E}_{K} \rightarrow X$ the universal bundle (principal $\tilde{G}_{K}$-bundle). Then, for any topological group $G$, any locally trivial G-bundle $\pi: E \rightarrow X$ is associated with $\tilde{\pi}_{K}$. 
Proof. From Proposition $8.6,\left(\tilde{\pi}_{K}, \tilde{G}_{K}, \tilde{P}_{K},\left[v_{0}\right]\right)$ is a strong holonomy reduction of itself. Thus from Theorem 8.7, $\left(\tilde{\pi}_{K}, \tilde{G}_{K}, \tilde{P}_{K},\left[v_{0}\right]\right)$ is an initial object in $\mathbf{C}\left(X, v_{0}, \Xi_{K}\right)$. On the other hand, from Theorem 5.2 in [14], there exists $\omega \in S F\left(\pi, U_{K}\right)_{G}$. Then $\left(\pi, G, P^{\omega}, u\right) \in \mathbf{C}\left(X, v_{0}, \Im_{K}\right)_{0}$, where $u \in E_{v_{0}}$. Thus there exists a unique morphism $(h, \rho):\left(\tilde{\pi}_{K}, \tilde{G}_{K}, \tilde{P}_{K},\left[v_{0}\right]\right) \rightarrow\left(\pi, G, P^{\omega}, u\right)$. Therefore $\pi$ is associated with $\tilde{\pi}_{K}$ by $\rho$.

\section{References}

[1] N. Abe, Parallel displacements in a category of certain topological spaces, Tensor (N.S.) 58 (1997), 83-91.

[2] James E. Arnold, Jr., Local to global theorems in the theory of Hurewicz fibrations, Trans. Amer. Math. Soc. 164 (1972), 179-188.

[3] A. Asada, Connection of topological vector bundles, Proc. Japan. Acad. 41 (1965), 919-922.

[4] A. Asada, Connection of topological fibre bundles, Proc. Japan. Acad. 42 (1966), 13-18.

[5] A. Asada, Connection of topological fibre bundles. II, Proc. Japan. Acad. 42 (1966), 231-236.

[6] Yu. Burago, M. Gromov, and G. Perel'man, A. D. Alexandrov spaces with curvature bounded below, Russian Math. Surveys. 47 (1992), 1-58.

[ 7 ] S. Echterhoff, H. Emerson, and H. J. Kim, $K K$-theoretic duality for proper twisted action, Math. Ann. 340 (2008), 839-873.

[ 8 ] M. Gromov, Metric structures for Riemannian and non-Riemannian spaces, Progress in Math. 152 Birkhäuser, Boston, 1999.

[ 9 ] W. Hurewicz and N. E. Steenrod, Homotopy relations in fibre spaces, Proc. Nat. Acad. Sci. U. S. A. 27 (1941), 60-64.

[10] D. Husemoller, Fibre bundles, Springer-Verlag, New York, 1994.

[11] G. G. Kasparov, Equivariant $K K$-theory and the Novikov conjecture, Invent. Math. 91 (1988), 147-201.

[12] S. Kobayashi and K. Nomizu, Foundations of differential geometry. Vol I, Interscience Publishers, New York-London, 1963.

[13] J. Kubarski and N. Teleman, Linear direct connections, Banach Center Publ., 76, Polish Acad. Sci., Warsaw, (2007), 425-436.

[14] J. Milnor, Construction of universal bundles. I, Ann. of Math. (2) 63 (1956), 272-284.

[15] A. Petrunin, Parallel transportation for Alexandrov space with curvature bounded below, geom. funct. anal., 8 (1998), 123-148.

[16] N. Teleman, Distance function, linear quasi-connections and Chern character, IHES Prepublications M/04/27, June 2004.

[17] G. W. Whitehead, Elements of homotopy theory, Springer-Verlag, New York, 1978.

Department of Mathematics

Tokyo University of Science

Kagurazaka 1-3, Shinjuku-ku

Tokyo 162-8601

Japan

e-mail: kitada@ma.kagu.tus.ac.jp 